\title{
A Spatial Stochastic Epidemic Model: Law of Large Numbers and Central Limit Theorem
}

\author{
S. Bowong* A. Emakoua ${ }^{\dagger} \quad$ E. Pardoux ${ }^{\ddagger}$
}

July 15,2020

\begin{abstract}
We consider an individual-based SIR stochastic epidemic model in continuous space. The evolution of the epidemic involves the rates of infection and cure of individuals. We assume that individuals move randomly on the two-dimensional torus according to independent Brownian motions. We define the empirical measures $\mu^{S, N}, \mu^{I, N}$ and $\mu^{R, N}$ which describe the evolution of the position of the susceptible, infected and removed individuals. We prove the convergence in propbability, as $N \rightarrow \infty$, of the sequence $\left(\mu^{S, N}, \mu^{I, N}\right)$ towards $\left(\mu^{S}, \mu^{I}\right)$ solution of a system of parabolic PDEs. We show that the sequence $\left(U^{N}=\sqrt{N}\left(\mu^{S, N}-\mu^{S}\right), V^{N}=\sqrt{N}\left(\mu^{I, N}-\mu^{I}\right)\right)$ converges in law, as $N \rightarrow \infty$, towards a Gaussian distribution valued process, solution of a system of linear PDEs with highly singular Gaussian driving processes. In the case where the individuals do not move we also define and study the law of large numbers and central limit theorem of the sequence $\left(\mu^{S, N}, \mu^{I, N}\right)$.
\end{abstract}

Keywords: Measure-valued process; Stochastic Epidemic model; Law of large numbers; Central limit theorem.

\section{Introduction}

Recent studies on homogeneous stochastic models of epidemics of Anderson and Britton [4], and Britton and Pardoux [8] show that deterministic models of epidemics are the law of large numbers limits (as the size of the population tends to $\infty$ ) of stochastic models, while the central limit theorem and moderate and large deviations (see [8] and [21]) give tools to accurately describe the gap between stochastic and deterministic models. However, the homogeneity hypothesis made in these models is not realistic since it means that when an infected individual infects a susceptible individual, this latter is uniformly chosen in the population, while in real life an infected individual infects a susceptible person who is geographically close to him. Hence the interest of modelling epidemics for a population distributed in a continuous space with or without displacement of the population. The case without displacement is important for populations of plants. The corresponding deterministic models are beginning to be well studied in the literature, see for example [3].

In this paper we study the law of large numbers and the central limit theorem of two stochastic SIR epidemic models for a population distributed on the two dimentional torus. The only difference between the two models is that in the first one the population is moving and in the second it does not move. More precisely we consider a population of size $\mathrm{N}$ distributed on the torus $\left(\mathbb{T}^{2}=\mathbb{R}^{2} / \mathbb{Z}^{2}\right)$ such that at any time each individual is either susceptible $(\mathrm{S})$, infectious $(\mathrm{I})$ or recovered $(\mathrm{R})$. Let $\mathrm{S}(\mathrm{t}), \mathrm{I}(\mathrm{t})$ and $\mathrm{R}(\mathrm{t})$ denote the number of individuals in the different states at time t. 
During the epidemic an individual $i$ moves on the torus according to the processes $\left\{X_{t}^{i}=\Pi\left(\widetilde{X}_{t}^{i}\right), t \geq 0\right\}$, where $\Pi$ is the canonical projection from $\mathbb{R}^{2}$ to $\mathbb{\mathbb { T }}^{2}, \widetilde{X}_{t}^{i}=X^{i}+\sqrt{2 \gamma} B_{t}^{i}$, with $\left\{X^{i}, 1 \leq i \leq N\right\}$ an independent and identically distributed family of random variables, globally independent of $\left\{B^{i}, 1 \leq i \leq N\right\}$, which in turn is a family of independent standard Brownian motions on the torus $(\gamma$ is a positive constant in the first model and is zero in the second). We assume at time $\mathrm{t}=0$ (for $\gamma \geq 0$ ), that the population consists of two classes $\mathrm{S}(0)$ and $\mathrm{I}(0)$ described as follows.

Let $A$ be an arbitrary Borel subset of $\mathbb{T}^{2}$ and $0<p \leq 1$, each individual $i$ is placed in $\mathbb{T}^{2}$ independently of the others at the position $X^{i}$. If $X^{i} \in A^{c}$ then the individual $i$ is susceptible and if $X^{i} \in A$, the individual $i$ is infected with probability $p$ and susceptible with probability $1-p$. This situation is modelled by empirical measures

$$
\begin{aligned}
& \mu_{0}^{S, N}=\frac{1}{N} \sum_{i=1}^{N}\left\{1_{A}\left(X^{i}\right)\left(1-\xi_{i}\right)+1_{A^{c}}\left(X^{i}\right)\right\} \delta_{X^{i}} \\
& \mu_{0}^{I, N}=\frac{1}{N} \sum_{i=1}^{N} 1_{A}\left(X^{i}\right) \xi_{i} \delta_{X^{i}} \\
& \mu_{0}^{N}=\mu_{0}^{S, N}+\mu_{0}^{I, N}=\frac{1}{N} \sum_{i=1}^{N} \delta_{X^{i}},
\end{aligned}
$$

where $\left\{\xi_{i}, 1 \leq i \leq N\right\}$ is a mutually independent family of $\operatorname{Ber}(p)$ random variables, globally independent of $\left\{X^{i}, 1 \leq i \leq N\right\}$.

Let $\mathrm{K}$ be a function defined by

$$
\begin{aligned}
K: & \mathbb{T}^{2} \times \mathbb{T}^{2} \rightarrow \mathbb{R}_{+} \\
& (x, y) \mapsto k\left(d_{\mathbb{T}^{2}}^{2}(x, y)\right)
\end{aligned}
$$

where $k: \mathbb{R}_{+} \rightarrow \mathbb{R}_{+}$is a non-increasing function which is non zero only in a neighbourhood of zero in such a way that by considering for any $x, y \in \mathbb{T}^{2}, d_{\mathbb{T}^{2}}(x, y)=\inf _{a \in \mathbb{Z}^{2}}\{\|x-y-a\|\}$, one has

$$
\forall x \in \mathbb{T}^{2}, y \in \operatorname{support}\{K(x, .)\} \text { iff } d_{\mathbb{W}^{2}}(x, y)=\|x-y\| .
$$

Let $E_{t}^{i}$ be the state of the individual $\mathrm{i}$ at time $\mathrm{t}, E_{t}^{i} \in\{S, I, R\}$ and $\beta$ a positive constant. In the homogeneous model [8], the rate of infectious contacts can be thought of as a product of a rate $c^{\prime}$ at which each infectious individual has contacts with others, and the probability $p^{\prime}$ that such a contact results in an infection given that the other person is susceptible, which happens with the probability $S_{t} / N$, where $\mathrm{N}$ is the total population size, because all the individuals have the same probability to be in contact with an infectious. However, in our case the rate $P_{j, i}$ at which an infectious $j$ has a contact with a susceptible $i$ depends upon the distance that separates them, so $P_{j, i}$ is proportional to $K\left(X_{t}^{i}, X_{t}^{j}\right)$, thus

- $P_{j, i}=\frac{\beta K\left(X_{t}^{i}, X_{t}^{j}\right)}{\sum_{l=1}^{N} K\left(X_{t}^{l}, X_{t}^{j}\right)}$

- An infectious individual $j$ has an infectious contact with susceptible individuals at the rate $\beta \frac{\sum_{i=1}^{N} K\left(X_{t}^{i}, X_{t}^{j}\right) 1_{\left\{E_{t}^{i}=S\right\}}}{\sum_{l=1}^{N} K\left(X_{t}^{l}, X_{t}^{j}\right)}$.

Hence infectious individuals have infectious contacts with susceptible individuals at the rate $\beta \sum_{j=1}^{N} \frac{\sum_{i=1}^{N} K\left(X_{t}^{i}, X_{t}^{j}\right) 1_{\left\{E_{t}^{i}=S\right\}}}{\sum_{l=1}^{N} K\left(X_{t}^{l}, X_{t}^{j}\right)} 1_{\left\{E_{t}^{j}=I\right\}}$.

The epidemic evolves according to the following rules:

- A susceptible $i$ becomes infected at time $\mathrm{t}$ at the rate $\beta 1_{\left\{E_{t}^{i}=S\right\}} \sum_{j=1}^{N} \frac{K\left(X_{t}^{i}, X_{t}^{j}\right)}{\sum_{l=1}^{N} K\left(X_{t}^{l}, X_{t}^{j}\right)} 1_{\left\{E_{t}^{j}=I\right\}}$

- Each infected individual cures at rate $\alpha$ independently of the others, of the number of infected and of the respective positions of the individuals.

The evolution of the numbers of susceptible, infected and removed individuals is described by the following equations. 


$$
\begin{aligned}
& S(t)=S(0)-P_{\text {inf }}\left(\beta \int_{0}^{t} \sum_{j=1}^{N} \frac{\sum_{i=1}^{N} K\left(X_{r}^{i}, X_{r}^{j}\right) 1_{\left\{E_{r}^{i}=S\right\}}}{\sum_{l=1}^{N} K\left(X_{r}^{l}, X_{r}^{j}\right)} 1_{\left\{E_{r}^{j}=I\right\}} d r\right) \\
& I(t)=I(0)+P_{\text {inf }}\left(\beta \int_{0}^{t} \sum_{j=1}^{N} \frac{\sum_{i=1}^{N} K\left(X_{r}^{i}, X_{r}^{j}\right) 1_{\left\{E_{r}^{i}=S\right\}}}{\sum_{l=1}^{N} K\left(X_{r}^{l}, X_{r}^{j}\right)} 1_{\left\{E_{r}^{j}=I\right\}} d r\right)-P_{c u}\left(\alpha \int_{0}^{t} \sum_{j=1}^{N} 1_{\left\{E_{r}^{j}=I\right\}} d r\right) \\
& R(t)=R(0)+P_{c u}\left(\alpha \int_{0}^{t} \sum_{j=1}^{N} 1_{\left\{E_{r}^{j}=I\right\}} d r\right)
\end{aligned}
$$

where $P_{\text {inf }}$ and $P_{c u}$ are two independent standard Poisson processes.

We now define the renormalized point processes, $\forall t>0$,

$$
\begin{aligned}
& \mu_{t}^{S, N}=\frac{1}{N} \sum_{i=1}^{N} 1_{\left\{E_{t}^{i}=S\right\}} \delta_{X_{t}^{i}} \\
& \mu_{t}^{I, N}=\frac{1}{N} \sum_{i=1}^{N} 1_{\left\{E_{t}^{i}=I\right\}} \delta_{X_{t}^{i}} \\
& \mu_{t}^{R, N}=\frac{1}{N} \sum_{i=1}^{N} 1_{\left\{E_{t}^{i}=R\right\}} \delta_{X_{t}^{i}} \\
& \mu_{t}^{N}=\frac{1}{N} \sum_{i=1}^{N} \delta_{X_{t}^{i}}=\mu_{t}^{S, N}+\mu_{t}^{I, N}+\mu_{t}^{R, N} .
\end{aligned}
$$

We first study the law of large numbers and the central limit theorem of the sequence $\left(\mu_{0}^{S, N}, \mu_{0}^{I, N}, \mu_{0}^{N}\right)_{N \geq 1}$, then we study the law of large numbers and the central limit theorem of the sequences $\left(\mu^{N}\right)_{N \geq 1}$ and $\left(\mu^{S, N}, \mu^{I, N}\right)_{N \geq 1}$ when $\gamma$ is a positive constant, and finally when $\gamma$ is zero.

The paper is organized as follows. In section 2 we recall some results that will be useful in the sequel. In sections 3 and 4 we study the law of large numbers and the central limit theorem of the sequence $\left(\mu_{0}^{S, N}, \mu_{0}^{I, N}, \mu_{0}^{N}\right)_{N \geq 1}$. In section 5 , for $\gamma>0$, we first establish the evolution equations of the measure-valued process $\mu^{S, N}$ and $\mu^{I, N}$ then we show that $\mu^{N}$ converges in probability as $N \rightarrow \infty$ towards the processes $\left\{\mu_{t}, t \geq 0\right\}$ where for each $t \geq 0, \mu_{t}$ is the law $X_{t}^{1}$ and finally we prove that $\left\{\left(\mu_{t}^{S, N}, \mu_{t}^{I, N}\right), t \geq 0\right\}$ converges in probability as $N \rightarrow \infty$ towards $\left(\mu^{S}, \mu^{I}\right)$ solution of a system of parabolic PDEs. In section 6 we study the convergence of the sequences $Z^{N}=\sqrt{N}\left(\mu^{N}-\mu\right)$ and $\left(U^{N}=\sqrt{N}\left(\mu^{S, N}-\mu\right), V^{N}=\sqrt{N}\left(\mu^{I, N}-\mu^{I}\right)\right)$. Finally in section 7 we assume that the individuals do not move $(\gamma=0)$ then we study the law of large numbers and the central limit theorem of the sequence $\left\{\left(\mu_{t}^{S, N}, \mu_{t}^{I, N}\right), N \geq 1, t \geq 0\right\}$.

\section{Notation:}

$-\mathcal{M}_{F}\left(\mathbb{T}^{2}\right)$ denotes the space of finite measures on $\mathbb{T}^{2}$.

$-C\left(\mathbb{T}^{2}\right)$ denotes the space of continuous functions on $\mathbb{T}^{2}$.

- $\forall \mu \in \mathcal{M}_{F}\left(\mathbb{T}^{2}\right)$ and $\varphi \in C\left(\mathbb{T}^{2}\right)$, we denote the integral $\int_{\mathbb{T}^{2}} \varphi(x) \mu(d x)$ by $(\mu, \varphi)$.

- We define the Fortet distance on $\mathcal{M}_{F}\left(\mathbb{T}^{2}\right)$ by $\forall \mu, \nu \in \mathcal{M}_{F}\left(\mathbb{T}^{2}\right)$

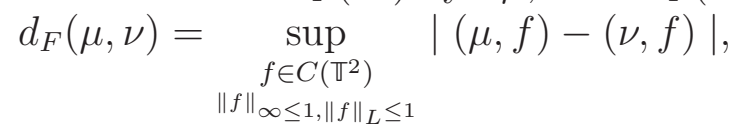

where $\|f\|_{L}=\sup _{x \neq y}(|f(x)-f(y)|) / d_{\mathbb{T}^{2}}(x, y)$.

This distance induces the topology of weak convergence, in other words the sequence of measures $\mu_{n}$ converges weakly towards $\mu$ if and only if $\lim _{n \rightarrow \infty} d_{F}\left(\mu_{n}, \mu\right)=0$

- In the following, the letter $C$ will denote a (constant) positive real number which can change from line to line.

- We equip $\mathcal{M}_{F}\left(\mathbb{T}^{2}\right)$ with the topology of weak convergence. 


\section{Preliminaries}

Definition 2.1. Sobolev spaces (see [1])

1) Let $m \in \mathbb{N}, p \in \mathbb{R}_{+}$the Sobolev space $W^{m, p}\left(\mathbb{T}^{2}\right)$ is defined by:

$W^{m, p}\left(\mathbb{T}^{2}\right)=\left\{\varphi \in L^{p}\left(\mathbb{T}^{2}\right): D^{\eta} \varphi \in L^{p}\left(\mathbb{T}^{2}\right), \forall \eta=\left(\eta_{1}, \eta_{2}\right) \in \mathbb{N}^{2}\right.$ such that $\left.|\eta|=\eta_{1}+\eta_{2} \leq m\right\}$ where $D^{\eta} \varphi$ is the weak derivative of the function $\varphi$ with respect to the multi-index $\eta$.

2) Let $s=n+\sigma$ with $n \in \mathbb{N}$ and $\sigma \in] 0,1\left[\right.$, the Sobolev space $W^{s, p}\left(\mathbb{T}^{2}\right)$ is defined as follows

$$
W^{s, p}\left(\mathbb{T}^{2}\right)=\left\{\varphi \in W^{m, p}\left(\mathbb{T}^{2}\right) / \sum_{|\eta|=n} \int_{\mathbb{T}^{2} \times \mathbb{T}^{2}} \frac{\mid D^{\eta}\left(\varphi(x)-\left.D^{\eta} \varphi\left(x^{\prime}\right)\right|^{2}\right.}{\left(d_{\mathbb{T}^{2}}\left(x, x^{\prime}\right)\right)^{2(1+\sigma)}} d x d x^{\prime}<\infty\right\} .
$$

Notice that for $p=2$ and $s \in \mathbb{R}_{+}, W^{s, 2}\left(\mathbb{T}^{2}\right)$ is denoted $H^{s}\left(\mathbb{T}^{2}\right)$ and is a Hilbert space.

Proposition 2.2. (see [7])

Consider the following sets of functions.

$$
\begin{aligned}
& \mathcal{A}_{1}=\left\{f_{n_{1}, n_{2}}^{1}\left(x_{1}, x_{2}\right)=2 \sin \left(\pi n_{1} x_{1}\right) \cos \left(\pi n_{2} x_{2}\right), n_{1}>0, n_{2}>0 \quad \text { even }\right\} \\
& \mathcal{A}_{2}=\left\{f_{n_{1}, n_{2}}^{2}\left(x_{1}, x_{2}\right)=2 \sin \left(\pi n_{1} x_{1}\right) \sin \left(\pi n_{2} x_{2}\right), n_{1}>0, n_{2}>0 \quad \text { even }\right\} \\
& \mathcal{A}_{3}=\left\{f_{n_{1}, n_{2}}^{3}\left(x_{1}, x_{2}\right)=2 \cos \left(\pi n_{1} x_{1}\right) \cos \left(\pi n_{2} x_{2}\right), n_{1}>0, n_{2}>0 \quad \text { even }\right\} \\
& \mathcal{A}_{4}=\left\{f_{n_{1}, n_{2}}^{4}\left(x_{1}, x_{2}\right)=2 \cos \left(\pi n_{1} x_{1}\right) \sin \left(\pi n_{2} x_{2}\right), n_{1}>0, n_{2}>0 \quad \text { even }\right\} \\
& \mathcal{A}_{5}=\left\{f_{n_{1}, 0}^{5}\left(x_{1}, x_{2}\right)=\sqrt{2} \cos \left(\pi n_{1} x_{1}\right), f_{n_{1}, 0}^{6}\left(x_{1}, x_{2}\right)=\sqrt{2} \sin \left(\pi n_{1} x_{1}\right), n_{1}>0 \text { even }\right\} \\
& \mathcal{A}_{6}=\left\{f_{0, n_{2}}^{7}\left(x_{1}, x_{2}\right)=\sqrt{2} \cos \left(\pi n_{2} x_{2}\right), f_{0, n_{2}}^{8}\left(x_{1}, x_{2}\right)=\sqrt{2} \sin \left(\pi n_{2} x_{2}\right), n_{2}>0 \text { even }\right\} .
\end{aligned}
$$

For any $\gamma>0$,

1) $\left\{f^{0}=1,\left(\mathcal{A}_{i}, i \in\{1,2,3,4\}\right), \mathcal{A}_{5}, \mathcal{A}_{6}\right\}$ are eigenfunctions of the operator $\gamma \triangle$ on $\mathbb{T}^{2}$, with eigenvalues $\left\{\lambda_{0}=0,-\lambda_{n_{1}, n_{2}}=-\gamma \pi^{2}\left(n_{1}^{2}+n_{2}^{2}\right),-\lambda_{n_{1}}=-\gamma \pi^{2} n_{1}^{2},-\lambda_{n_{2}}=-\gamma \pi^{2} n_{2}^{2}\right\}$ respectively, they form an orthonormal basis of $\mathbb{L}^{2}\left(\mathbb{T}^{2}\right)$.

2) $\left\{\rho^{0}=1,\left\{\rho_{n_{1}, n_{2}}^{i, s}=\frac{f_{n_{1}, n_{2}}^{i}}{\left(1+\gamma \pi^{2}\left(n_{1}^{2}+n_{2}^{2}\right)\right)^{\frac{s}{2}}}, i \in\{1,2,3,4\}\right\},\left\{\rho_{n_{1}, 0}^{i, s}=\frac{f_{n_{1}, 0}^{i}}{\left(1+\gamma \pi^{2} n_{1}^{2}\right)^{\frac{s}{2}}} i \in\{5,6\}\right\}\right.$,

$\left.\left\{\rho_{0, n_{2}}^{i, s}=\frac{f_{0, n_{2}}^{i}}{\left(1+\gamma \pi^{2} n_{2}^{2}\right)^{\frac{s}{2}}}, i \in\{7,8\}\right\}\right\}$ is an orthonormal basis of $H^{s}\left(\mathbb{T}^{2}\right)$.

Proposition 2.3. Parseval identity (see the lemma 6.52 [1])

For any $s>0, \varphi \in H^{s}\left(\mathbb{T}^{2}\right), A \in H^{-s}\left(\mathbb{T}^{2}\right)$

$\|A\|_{H^{-s}}=\sup _{\varphi \neq 0, \varphi \in H^{s}} \frac{|(A, \varphi)|}{\|\varphi\|_{H^{s}}}$

$$
\begin{aligned}
\|A\|_{H^{-s}}^{2} & =\left|\left(A, \rho^{0}\right)\right|^{2}+\sum_{\substack{i \in\{5,6\} \\
n_{1}>0, \text { even }}}\left|\left(A, \rho_{n_{1}, 0}^{i, s}\right)\right|^{2}+\sum_{\substack{i \in\{7,8\} \\
n_{2}>0, \text { even }}}\left|\left(A, \rho_{0, n_{2}}^{i, s}\right)\right|^{2}+\sum_{\substack{i \in\{1,2,3,4\} \\
n_{1}>0, n_{2}>0, \text { even }}}\left|\left(A, \rho_{n_{1}, n_{2}}^{i, s}\right)\right|^{2} \\
& =\sum_{i, n_{1}, n_{2}}\left|\left(A, \rho_{n_{1}, n_{2}}^{i, s}\right)\right|^{2}
\end{aligned}
$$

$$
\begin{aligned}
\|\varphi\|_{H^{s}}^{2}= & \left(\varphi, f^{0}\right)_{L^{2}}^{2}+\sum_{\substack{i \in\{5,6\} \\
n_{1}>0, \text { even }}}\left(1+\gamma \pi^{2} n_{1}^{2}\right)^{s}\left(\varphi, f_{n_{1}, 0}^{i}\right)_{L^{2}}^{2}+\sum_{\substack{i \in\{7,8\} \\
n_{2}>0, \text { even }}}\left(1+\gamma \pi^{2} n_{2}^{2}\right)^{s}\left(\varphi, f_{0, n_{2}}^{i}\right)_{L^{2}}^{2} \\
& +\sum_{\substack{i \in\{1,2,3,4\} \\
n_{1}>0, n_{2}>0, \text { even }}}\left(1+\gamma \pi^{2}\left(n_{1}^{2}+n_{2}^{2}\right)\right)^{s}\left(\varphi, f_{n_{1}, n_{2}}^{i}\right)_{L^{2}}^{2} \\
= & \sum_{i, n_{1}, n_{2}}\left(1+\gamma \pi^{2}\left(n_{1}^{2}+n_{2}^{2}\right)\right)^{s}\left(\varphi, f_{n_{1}, n_{2}}^{i}\right)_{L^{2}}^{2}
\end{aligned}
$$

where $(., .)_{L^{2}}$ denote the scalar product in $L^{2}\left(\mathbb{T}^{2}\right)$ and $(.,$.$) is the duality product.$

Proposition 2.4. Sobolev injection (see [25] page 22)

1) If $s>k+1$ with $k \in \mathbb{N}$ then $H^{s}\left(\mathbb{T}^{2}\right) \subset C^{k}\left(\mathbb{T}^{2}\right)$ and $\forall \varphi \in H^{s}\left(\mathbb{T}^{2}\right), \exists C(s)>0$ such that $\left\|D^{\eta} \varphi\right\|_{\infty} \leq C(s)\|\varphi\|_{H^{s}}, \forall|\eta| \leq k$.

2) If $s>1$ then $H^{s}\left(\mathbb{T}^{2}\right)$ is a Banach algebra, ie $\exists C>0 ; \forall u, v \in H^{s}\left(\mathbb{T}^{2}\right)$, uv $\in H^{s}\left(\mathbb{T}^{2}\right)$ and $\|u v\|_{H^{s}} \leq C\|u\|_{H^{s}}\|v\|_{H^{s}}$. 
Proposition 2.5. Description of the Contraction Semigroup (see [7] and [15]). Let $\gamma>0$,

1) The operator $\gamma \triangle$ is selfadjoint, unbounded on $L^{2}\left(\mathbb{T}^{2}\right)$ and it is the infinitesimal generator of a semigroup $\Upsilon(t)=e^{\gamma t \triangle}$, furthemore $\forall \varphi \in L^{2}\left(\mathbb{T}^{2}\right)$,

$$
\Upsilon(t) \varphi=\sum_{i, n_{1}, n_{2}} \exp \left(-\lambda_{n_{1}, n_{2}} t\right)\left(\varphi, f_{n_{1}, n_{2}}^{i}\right)_{L^{2}} f_{n_{1}, n_{2}}^{i} .
$$

2) $\forall s<0$ (resp. $s>0) \Upsilon(t)$ and $\gamma \triangle$ have extension (resp. restriction) to $H^{s}\left(\mathbb{T}^{2}\right)$ such that $\Upsilon(t)$ is a contraction semigroup, stronly-continuous, and bounded on $H^{s}\left(\mathbb{T}^{2}\right)$ with $|\Upsilon(t)|_{\mathcal{L}\left(H^{s}\left(\mathbb{T}^{2}\right)\right)} \leq 1$, where $\mathcal{L}\left(H^{s}\left(\mathbb{T}^{2}\right)\right)$ is the space of linear continuous operator on $H^{s}\left(\mathbb{T}^{2}\right)$.

Lemma 2.6. (see [15]) $\forall s>0, \forall \varphi \in H^{s}\left(\mathbb{T}^{2}\right)$, resp. $\forall \varphi \in L^{\infty}\left(\mathbb{T}^{2}\right)$, from (2.1), we have

$$
\|\Upsilon(t) \varphi\|_{H^{s}} \leq\|\varphi\|_{H^{s}} \text { and }\|\Upsilon(t) \varphi\|_{\infty} \leq\|\varphi\|_{\infty} .
$$

Lemma 2.7. (Lemma 2.5 of [9])

Suppose that $(\mathbb{D}, d)$ is a separable metric space and let $\left(\hat{P}_{n}\right)_{n}$ denote a sequence of random probability measures on $(\mathbb{D}, \mathcal{D})$ defined on a probability space $(\Omega, \mathcal{A}, \mathbb{P})$. Then

$$
\int_{\mathbb{D}} f(x) d \hat{P}_{n}(x) \stackrel{\mathbb{P}}{\rightarrow} \int_{\mathbb{D}} f(x) d P(x),
$$

for any $f$ bounded and Lipschitz if and only if $d_{F}\left(\hat{P}_{n}, P\right) \stackrel{\mathbb{P}}{\rightarrow} 0$.

Let $T>0$. Let $F$ be a separable and reflexive Banach space included (with density and continuous injection) in a Hilbert space $\mathrm{H}$. We identify $\mathrm{H}$ with its dual. So $F \subset H \subset F^{\prime}$, where $F^{\prime}$ is the dual of $F$. Let $(A(t, .))_{\{t \in] 0, T[\}}$ be a family of linear operators from $F$ to $F^{\prime}$, such that:

(1) $\theta \longrightarrow(A(t, u+\theta v), w)$ is continuous from $\mathbb{R}$ to $\mathbb{R}, \forall u, v, w \in F$

(2) $\exists \delta>0,\|A(t, u)\|_{F^{\prime}} \leq \delta\|u\|_{F}, \forall u \in F$

(3) $\exists \sigma_{1}>0, \sigma_{2} \in \mathbb{R},(A(t, u), u)+\sigma_{2}\|u\|_{H}^{2} \geq \sigma_{1}\|u\|_{F}^{2}, \forall u \in F$

(4) $\forall u \in F, t \longrightarrow A(t, u)$ est Lebesgue-mesurable with values in $F^{\prime}$,

where $(.,$.$) is a duality product between F^{\prime}$ and $F$.

Proposition 2.8. (see Theorem 1.1 page 81 in [20])

Let $\left(\Omega, \mathcal{F}_{0}, \mathbb{P}\right)$ be a probability space, let $(A(t, .))_{\{t \in] 0, T[\}}$ be a family of operators from $F$ to $F^{\prime}$ which satisfy (1), (2), (3) and (4). For $u(0) \in L^{2}\left(\Omega, \mathcal{F}_{0}, \mathbb{P}, H\right), f=f_{1}+f_{2}$ with

- $f_{1} \in L^{2}\left(\Omega, L^{1}(0, T, H)\right)$, non-anticipative,

$-f_{2} \in L^{2}(\Omega \times] 0, T[, F)$, non-anticipative,

and $\left(M_{t}\right)_{0 \leq t \leq T}$ a continuous square-integrable martingale with values in $H$, the equation

$$
d u(t)+A(u(t)) d t=f(t) d t+d M(t), t \in[0, T] \text { with } u(0)=u_{0},
$$

admits a unique solution $u \in L^{2}(\Omega \times] 0, T[, F) \cap L^{2}(\Omega, C([0, T], H))$.

\section{Law of Large Numbers of Initial Measures}

Recall that $\mu_{0}^{S, N}=\frac{1}{N} \sum_{i=1}^{N}\left\{1_{A}\left(X^{i}\right)\left(1-\xi_{i}\right)+1_{A^{c}}\left(X^{i}\right)\right\} \delta_{X^{i}}, \mu_{0}^{I, N}=\frac{1}{N} \sum_{i=1}^{N} 1_{A}\left(X^{i}\right) \xi_{i} \delta_{X^{i}}$ and $\mu_{0}^{N}=\mu_{0}^{S, N}+\mu_{0}^{I, N}=\frac{1}{N} \sum_{i=1}^{N} \delta_{X^{i}}$ where $\left\{X^{i}, 1 \leq i \leq N\right\}$ is an independent an identically distributed family of random variables, globally independent of $\left\{\xi_{i}, 1 \leq i \leq N\right\}$, which in turn is a mutually independent family of $\operatorname{Ber}(p)$.

The following is assumed to be hold throughout this paper.

Assumption (H0): The law $\nu$ of $X^{1}$ is abosuletly continuous with respect to the Lebesgue measure and its density $g$ satisfies:

there exists $\delta_{1}>0, \delta_{2}>0$ such that $\delta_{1} \leq g(x) \leq \delta_{2}, \forall x \in \mathbb{T}^{2}$. 
Theorem 3.1. The sequence $\left(\mu_{0}^{S, N}, \mu_{0}^{I, N}, \mu_{0}^{N}\right)_{N \geq 1}$ converges a.s towards $\left(\mu_{0}^{S}, \mu_{0}^{I}, \mu_{0}\right)$ in $\left(\mathcal{M}_{F}\left(\mathbb{T}^{2}\right)\right)^{3}$, where $\mu_{0}^{S}(d x)=\left\{(1-p) 1_{A}(x)+1_{A^{c}}(x)\right\} \nu(d x), \mu_{0}^{I}(d x)=p 1_{A}(x) \nu(d x)$ and $\mu_{0}(d x)=\nu(d x)$.

Proof. All we need is to prove that for any $\varphi, \psi, \phi \in C\left(\mathbb{T}^{2}\right)$ the sequence $\left(\left(\mu_{0}^{S, N}, \varphi\right),\left(\mu_{0}^{I, N}, \psi\right),\left(\mu_{0}^{N}, \phi\right)\right)_{N \geq 1}$ converges a.s towards $\left(\left(\mu_{0}^{S}, \varphi\right),\left(\mu_{0}^{I}, \psi\right),\left(\mu_{0}, \phi\right)\right)$. Let $\varphi, \psi, \phi \in C\left(\mathbb{T}_{2}\right)$, we have

$$
\begin{aligned}
\left(\mu_{0}^{S, N}, \varphi\right) & =\frac{1}{N} \sum_{i=1}^{N}\left[1_{A}\left(X^{i}\right)\left(1-\xi_{i}\right)+1_{A^{c}}\left(X^{i}\right)\right] \varphi\left(X^{i}\right) \\
\left(\mu_{0}^{I, N}, \psi\right) & =\frac{1}{N} \sum_{i=1}^{N} 1_{A}\left(X^{i}\right) \xi_{i} \psi\left(X^{i}\right) \\
\left(\mu_{0}^{N}, \phi\right) & =\frac{1}{N} \sum_{i=1}^{N} \phi\left(X^{i}\right) .
\end{aligned}
$$

Furthemore, according to the law of large numbers

$$
\begin{aligned}
& \left(\mu_{0}^{S, N}, \varphi\right) \stackrel{a . s}{\longrightarrow} \mathbb{E}\left(\left(1_{A}\left(X^{1}\right)\left(1-\xi_{1}\right)+1_{A^{c}}\left(X^{1}\right)\right) \varphi\left(X^{1}\right)\right)=\int_{\mathbb{T}^{2}}\left\{(1-p) 1_{A}(x)+1_{A^{c}}(x)\right\} \varphi(x) \nu(d x), \\
& \left(\mu_{0}^{I, N}, \psi\right) \stackrel{a . s}{\longrightarrow} \mathbb{E}\left(1_{A}\left(X^{1}\right) \xi_{1} \psi\left(X^{1}\right)\right)=p \int_{A} \psi(x) \nu(d x), \\
& \left(\mu_{0}^{N}, \phi\right) \stackrel{a . s}{\longrightarrow} \mathbb{E}\left(\phi\left(X^{1}\right)\right)=\int_{\mathbb{T}^{2}} \phi(x) \nu(d x) .
\end{aligned}
$$

Thus

$$
\begin{aligned}
&\left(\left(\mu_{0}^{S, N}, \varphi\right),\left(\mu_{0}^{I, N}, \psi\right),\left(\mu_{0}^{N}, \phi\right)\right) \stackrel{a . s}{\longrightarrow}\left((1-p) \int_{A} \varphi(x) \nu(d x)+\int_{A^{c}} \varphi(x) \nu(d x)\right. \\
&\left.p \int_{A} \psi(x) \nu(d x), \int_{\mathbb{T}^{2}} \phi(x) \nu(d x)\right) .
\end{aligned}
$$

\section{Central Limit Theorem of Initial Measures}

We define $\left.U_{0}^{N}=\sqrt{N}\left(\mu_{0}^{S, N}-\mu_{0}^{S}\right), V_{0}^{N}=\sqrt{N}\left(\mu_{0}^{I, N}-\mu_{0}^{I}\right)\right)$ and $Z_{0}^{N}=\sqrt{N}\left(\mu_{0}^{N}-\mu_{0}\right)$. In this section we study the convergence of the sequence $\left(U_{0}^{N}, V_{0}^{N}, Z_{0}^{N}\right)$ in $\left(H^{-s}\left(\mathbb{T}^{2}\right)\right)^{3}$, as $N \rightarrow \infty$, with $\mathrm{s}>1$.

Proposition 4.1. For any $s>1$, there exists $C_{1}, C_{2}, C_{3}>0$ such that

$$
\sup _{N \geq 1} \mathbb{E}\left(\left\|Z_{0}^{N}\right\|_{H^{-s}}^{2}\right)<C_{1} ; \quad \sup _{N \geq 1} \mathbb{E}\left(\left\|U_{0}^{N}\right\|_{H^{-s}}^{2}\right)<C_{2} \text { and } \sup _{N \geq 1} \mathbb{E}\left(\left\|V_{0}^{N}\right\|_{H^{-s}}^{2}\right)<C_{3} .
$$

Proof. We only prove that $\sup _{N \geq 1} \mathbb{E}\left(\left\|V_{0}^{N}\right\|_{H^{-s}}^{2}\right)<C_{3}$. The other estimates follow by a similar argument. Since $1_{A}\left(X_{j}\right) \xi_{j} \delta_{X_{j}}$ are i.i.d with law $\mu_{0}^{I}$, from assumption (H0) and Lemma 8.1 in the Appendix below, we have

$$
\begin{aligned}
\mathbb{E}\left(\left\|V_{0}^{N}\right\|_{H^{-s}}^{2}\right) & =\mathbb{E}\left(\sum_{i, n_{1}, n_{2}}\left(V_{0}^{N}, \rho_{n_{1}, n_{2}}^{i, s}\right)^{2}\right) \\
& =N \sum_{i, n_{1}, n_{2}} \mathbb{E}\left(\left(\left(\mu_{0}^{I, N}, \rho_{n_{1}, n_{2}}^{i, s}\right)-\left(\mu_{0}^{I}, \rho_{n_{1}, n_{2}}^{i, s}\right)\right)^{2}\right) \\
& =\frac{1}{N} \sum_{i, n_{1}, n_{2}} \mathbb{E}\left(\left[\sum_{j=1}^{N}\left[1_{A}\left(X_{j}\right) \xi_{j} \rho_{n_{1}, n_{2}}^{i, s}\left(X_{j}\right)-\left(\mu_{0}^{I}, \rho_{n_{1}, n_{2}}^{i, s}\right)\right]\right]^{2}\right) \\
& =\frac{1}{N} \sum_{i, n_{1}, n_{2}} \sum_{j=1}^{N} \mathbb{E}\left(\left[1_{A}\left(X_{j}\right) \xi_{j} \rho_{n_{1}, n_{2}}^{i, s}\left(X_{j}\right)-\left(\mu_{0}^{I}, \rho_{n_{1}, n_{2}}^{i, s}\right)\right]^{2}\right) \\
& \leq \frac{1}{N} \sum_{i, n_{1}, n_{2}} \sum_{j=1}^{N} \mathbb{E}\left(\left[1_{A}\left(X_{j}\right) \xi_{j} \rho_{n_{1}, n_{2}}^{i, s}\left(X_{j}\right)\right]^{2}\right) \\
& \leq p \int_{A} \sum_{i, n_{1}, n_{2}}\left(\rho_{n_{1}, n_{2}}^{i, s}\right)^{2}(x) g(x) d x \leq p \delta_{2} C \quad \text { if } \mathrm{s}>1 .
\end{aligned}
$$


Let us give now the main result of this section.

Theorem 4.2. For any $s>1$, the sequence $\left(U_{0}^{N}, V_{0}^{N}, Z_{0}^{N}\right)_{N \geq 1}$ converges in law in $\left(H^{-s}\left(\mathbb{T}^{2}\right)\right)^{3}$ towards $\left(U_{0}, V_{0}, Z_{0}\right)$ where $\forall \varphi, \psi, \phi \in H^{s}\left(\mathbb{T}^{2}\right),\left(\left(U_{0}, \varphi\right),\left(V_{0}, \psi\right),\left(Z_{0}, \phi\right)\right)$ is a Gaussian vector which satisfies:

$$
\begin{aligned}
\left(U_{0}, \varphi\right)= & W_{1}\left[\varphi \sqrt{g}\left\{(1-p) 1_{A}+1_{A^{c}}\right\}\right]-(1-p) W_{1}(\sqrt{g}) \int_{A} \varphi(x) g(x) d x-W_{1}(\sqrt{g}) \int_{A^{c}} \varphi(x) g(x) d x \\
& +W_{2}\left(1_{A} \varphi \sqrt{\left(p-p^{2}\right) g}\right) \\
\left(V_{0}, \psi\right)= & p W_{1}\left(1_{A} \psi \sqrt{g}\right)-p W_{1}(\sqrt{g}) \int_{A} \psi(x) g(x) d x-W_{2}\left(1_{A} \psi \sqrt{\left(p-p^{2}\right) g}\right) \\
\left(Z_{0}, \phi\right)= & W_{1}(\phi \sqrt{g})-W_{1}(\sqrt{g})\left(\int_{\mathbb{T}^{2}} \phi(x) g(x) d x\right)
\end{aligned}
$$

where $W_{1}, W_{2}$ are mutually independent two dimentional white noises.

\subsection{Proof of Theorem 4.2}

We first prove the tightness of the sequence $\left(U_{0}^{N}, V_{0}^{N}, Z_{0}^{N}\right)_{N \geq 1}$, then identify the limit.

\subsubsection{Tightness of $\left(U_{0}^{N}, V_{0}^{N}, Z_{0}^{N}\right)_{N \geq 1}$}

Proposition 4.3. For any $s>1$, the sequences $\left(U_{0}^{N}\right)_{N \geq 1} ;\left(V_{0}^{N}\right)_{N \geq 1}$ and $\left(Z_{0}^{N}\right)_{N \geq 1}$ are tight in $H^{-s}$.

Proof. The tightness of $\left(U_{0}^{N}\right)_{N \geq 1}$ in $H^{-s}$ follows readily from the fact that

$$
\sup _{N \geq 1} \mathbb{E}\left(\left\|U_{0}^{N}\right\|_{H^{-s}}^{2}\right) \leq C_{1} .
$$

Indeed, since $\forall 1<s^{\prime}<s$, the embedding $H^{-s^{\prime}}\left(\mathbb{T}^{2}\right) \hookrightarrow H^{-s}\left(\mathbb{T}^{2}\right)$ is compact (see Theorem 1.69 page 47 of [5]) then $B_{H^{-s^{\prime}}}=\left\{\mu \in H^{-s^{\prime}} ;\|\mu\|_{H^{-s^{\prime}}} \leq R\right\}$ is a compact subset of $H^{-s}$.

Thus $\mathbb{P}\left(U_{0}^{N} \notin B_{H^{-s^{\prime}}}\right)=\mathbb{P}\left(\left\|U_{0}^{N}\right\|_{H^{-s^{\prime}}}>R\right)$

$$
\leq \frac{1}{R^{2}} \mathbb{E}\left(\left\|U_{0}^{N}\right\|_{H^{-s^{\prime}}}^{2}\right) \leq \frac{C_{1}}{R^{2}} .
$$

So by choosing $\mathrm{R}$ large enough we get the result.

The tightness of $\left(V_{0}^{N}\right)_{N \geq 1}$ and $\left(Z_{0}^{N}\right)_{N \geq 1}$ are obtained by similar arguments.

From Proposition 4.3 we deduce that the sequence $\left(U_{0}^{N}, V_{0}^{N}, Z_{0}^{N}\right)_{N \geq 1}$ is tight in $\left(H^{-s}\right)^{3}$, thus by Prokhorov's theorem there exists a subsequence still denoted $\left(U_{0}^{N}, V_{0}^{N}, Z_{0}^{N}\right)_{N \geq 1}$ which converges in law towards $\left(U_{0}, V_{0}, Z_{0}\right)$ in $\left(H^{-s}\right)^{3}$.

\subsubsection{Gaussian caracter and expressions of $\left(\left(U_{0}, \varphi\right),\left(V_{0}, \psi\right),\left(Z_{0}, \phi\right)\right)$ using white noises}

Let $\varphi, \psi, \phi \in H^{s}$, let us first compute the values of $\operatorname{Var}\left(\left(U_{0}, \varphi\right)\right) ; \operatorname{Var}\left(\left(V_{0}, \psi\right)\right)$; $\operatorname{Var}\left(\left(Z_{0}, \phi\right)\right)$; $\operatorname{Cov}\left(\left(U_{0}, \varphi\right),\left(V_{0}, \psi\right)\right) ; \operatorname{Cov}\left(\left(U_{0}, \varphi\right),\left(Z_{0}, \phi\right)\right)$; and $\operatorname{Cov}\left(\left(V_{0}, \psi\right),\left(Z_{0}, \phi\right)\right)$.

- Computation of $\operatorname{Var}\left(\left(U_{0}, \varphi\right)\right)$. We have

$$
\begin{aligned}
\left(U_{0}^{N}, \varphi\right) & =\sqrt{N}\left[\left(\mu_{0}^{S, N}, \varphi\right)-\left(\mu_{0}^{S}, \varphi\right)\right] \\
& =\sqrt{N}\left[\frac{1}{N} \sum_{i=1}^{N}\left\{1_{A}\left(X^{i}\right)\left(1-\xi_{i}\right)+1_{A^{c}}\left(X^{1}\right)\right\} \varphi\left(X^{i}\right)-(1-p) \int_{A} \varphi(x) d x-\int_{A^{c}} \varphi(x) d x\right],
\end{aligned}
$$

and

$$
\begin{aligned}
& \operatorname{Var}\left[\left\{1_{A}\left(X^{1}\right)\left(1-\xi_{1}\right)+1_{A^{c}}\left(X^{1}\right)\right\} \varphi\left(X_{1}\right)\right]= \\
& =\operatorname{Var}\left[1_{A}\left(X^{1}\right)\left(1-\xi_{1}\right) \varphi\left(X^{1}\right)\right]+\operatorname{Var}\left(1_{A^{c}}\left(X^{1}\right) \varphi\left(X^{1}\right)\right)+2 \operatorname{Cov}\left[1_{A}\left(X^{1}\right)\left(1-\xi_{1}\right) \varphi\left(X^{1}\right), 1_{A^{c}}\left(X^{1}\right) \varphi\left(X^{1}\right)\right] \\
& =\operatorname{Var}\left[1_{A}\left(X^{1}\right)\left(1-\xi_{1}\right) \varphi\left(X^{1}\right)\right]+\operatorname{Var}\left(1_{A^{c}}\left(X^{1}\right) \varphi\left(X^{1}\right)\right)-2 \mathbb{E}\left[1_{A}\left(X^{1}\right)\left(1-\xi_{1}\right) \varphi\left(X^{1}\right)\right] \mathbb{E}\left(1_{A^{c}}\left(X^{1}\right) \varphi\left(X^{1}\right)\right)
\end{aligned}
$$




$$
\begin{aligned}
& =(1-p) \int_{A} \varphi^{2}(x) g(x) d x-(1-p)^{2}\left(\int_{A} \varphi(x) g(x) d x\right)^{2}+\int_{A^{c}} \varphi^{2}(x) g(x) d x-\left(\int_{A^{c}} \varphi(x) g(x) d x\right)^{2} \\
& -2(1-p) \int_{A} \varphi(x) g(x) d x \int_{A^{c}} \varphi(x) g(x) d x \\
& =\alpha_{p} .
\end{aligned}
$$

So according to the central limit theorem

$$
\left(U_{0}^{N}, \varphi\right) \stackrel{L}{\rightarrow}\left(U_{0}, \varphi\right) \text { where }\left(U_{0}, \varphi\right) \rightsquigarrow \mathcal{N}\left(0, \alpha_{p}\right) .
$$

- Computation of $\operatorname{Var}\left(\left(V_{0}, \psi\right)\right)$. One has

$$
\begin{aligned}
& \left(V_{0}^{N}, \psi\right)=\sqrt{N}\left(\left(\mu_{0}^{I, N}, \psi\right)-\left(\mu_{0}^{I}, \psi\right)\right) \text { and } \\
& \operatorname{Var}\left[1_{A}\left(X^{1}\right) \xi_{1} \psi\left(X^{1}\right)\right]=p \int_{A} \psi^{2}(x) g(x) d x-p^{2}\left(\int_{A} \psi(x) g(x) d x\right)^{2}=\beta_{p} .
\end{aligned}
$$

So according to the central limit theorem

$$
\left(V_{0}^{N}, \psi\right) \stackrel{L}{\rightarrow}\left(V_{0}, \psi\right) \text { where }\left(V_{0}, \psi\right) \rightsquigarrow \mathcal{N}\left(0, \beta_{p}\right) .
$$

- Computation of $\operatorname{Var}\left(\left(Z_{0}, \phi\right)\right)$. According to central limit theorem

$$
\left(Z_{0}^{N}, \phi\right) \stackrel{L}{\rightarrow}\left(Z_{0}, \phi\right) \text { where }\left(Z_{0}, \phi\right) \rightsquigarrow N\left(0, \sigma^{2}=\int_{\mathbb{T}_{2}} \phi^{2}(x) g(x) d x-\left(\int_{\mathbb{T}^{2}} \phi(x) g(x) d x\right)^{2}\right) \text {. }
$$

- Computation of $\operatorname{Cov}\left(\left(U_{0}, \varphi\right),\left(V_{0}, \psi\right)\right), \operatorname{Cov}\left(\left(U_{0}, \varphi\right),\left(Z_{0}, \phi\right)\right)$ and $\operatorname{Cov}\left(\left(V_{0}, \psi\right),\left(Z_{0}, \phi\right)\right)$.

As $\operatorname{Cov}\left(\left(U_{0}, \varphi\right),\left(V_{0}, \psi\right)\right)=\frac{1}{2}\left[\operatorname{Var}\left(\left(U_{0}, \varphi\right)+\left(V_{0}, \psi\right)\right)-\operatorname{Var}\left(\left(U_{0}, \varphi\right)\right)-\operatorname{Var}\left(\left(V_{0}, \psi\right)\right)\right]$,

so we need to compute $\operatorname{Var}\left(\left(U_{0}, \varphi\right)+\left(V_{0}, \psi\right)\right), \operatorname{Var}\left(\left(U_{0}, \varphi\right)+\left(Z_{0}, \phi\right)\right)$ and $\operatorname{Var}\left(\left(V_{0}, \psi\right)+\left(Z_{0}, \phi\right)\right)$. We have

$$
\begin{aligned}
\left(U_{0}^{N}, \varphi\right) & +\left(V_{0}^{N}, \psi\right)+\left(Z_{0}^{N}, \phi\right)=\sqrt{N}\left\{\frac { 1 } { N } \sum _ { i = 1 } ^ { N } \left[\left\{1_{A}\left(X^{i}\right)\left(1-\xi_{i}\right)+1_{A^{c}}\left(X^{i}\right)\right\} \varphi\left(X^{i}\right)+1_{A}\left(X^{i}\right) \xi_{i} \psi\left(X^{i}\right)\right.\right. \\
& \left.\left.+\phi\left(X^{i}\right)\right]-(1-p) \int_{A} \varphi(x) g(x) d x-\int_{A^{c}} \varphi(x) g(x) d x-p \int_{A} \psi(x) g(x) d x-\int_{\mathbb{T}^{2}} \phi(x) g(x) d x\right\},
\end{aligned}
$$

and

$$
\begin{aligned}
\operatorname{Var}\left[\left\{1_{A}\left(X^{1}\right)\right.\right. & \left.\left.\left(1-\xi_{1}\right)+1_{A^{c}}\left(X^{1}\right)\right\} \varphi\left(X^{1}\right)+1_{A}\left(X^{1}\right) \xi_{1} \psi\left(X^{1}\right)+\phi\left(X^{1}\right)\right] \\
= & \alpha_{p}+\beta_{p}+\sigma^{2}+2 \operatorname{Cov}\left[\left\{1_{A}\left(X^{1}\right)\left(1-\xi_{1}\right)+1_{A^{c}}\left(X^{1}\right)\right\} \varphi\left(X^{1}\right), 1_{A}\left(X^{1}\right) \xi_{1} \psi\left(X^{1}\right)\right] \\
& +2 \operatorname{Cov}\left[\left\{1_{A}\left(X^{1}\right)\left(1-\xi_{1}\right)+1_{A^{c}}\left(X^{1}\right)\right\} \varphi\left(X^{1}\right), \phi\left(X^{1}\right)\right]+2 \operatorname{Cov}\left[1_{A}\left(X^{1}\right) \xi_{1} \psi\left(X^{1}\right), \phi\left(X^{1}\right)\right] .
\end{aligned}
$$

Furthemore since $\left.\left\{1_{A}\left(X^{1}\right)\left(1-\xi_{1}\right)+1_{A^{c}}\left(X^{1}\right)\right\} \varphi\left(X^{1}\right) 1_{A}\left(X^{1}\right) \xi_{1} \psi\left(X^{1}\right)\right)=0$ a.s,

$$
\begin{aligned}
\operatorname{Cov}\left[\left\{1_{A}\left(X^{1}\right)(1\right.\right. & \left.\left.\left.-\xi_{1}\right)+1_{A^{c}}\left(X^{1}\right)\right\} \varphi\left(X^{1}\right), 1_{A}\left(X^{1}\right) \xi_{1} \psi\left(X^{1}\right)\right] \\
& =-\mathbb{E}\left[\left\{1_{A}\left(X^{1}\right)\left(1-\xi_{1}\right)+1_{A^{c}}\left(X^{1}\right)\right\} \varphi\left(X^{1}\right)\right] \mathbb{E}\left(1_{A}\left(X^{1}\right) \xi_{1} \psi\left(X^{1}\right)\right) \\
& =-p(1-p) \int_{A} \varphi(x) g(x) d x \int_{A} \psi(x) g(x) d x-p \int_{A^{c}} \varphi(x) g(x) d x \int_{A} \psi(x) g(x) d x \\
& =\gamma_{p} .
\end{aligned}
$$

On the other hand:

$$
\begin{aligned}
\operatorname{Cov}\left[\left\{1_{A}\left(X^{1}\right)\right.\right. & \left.\left.\left(1-\xi_{1}\right)+1_{A^{c}}\left(X^{1}\right)\right\} \varphi\left(X^{1}\right), \phi\left(X^{1}\right)\right] \\
= & (1-p) \int_{A} \varphi(x) \phi(x) g(x) d x+(p-1) \int_{A} \varphi(x) g(x) d x \int_{\mathbb{T}^{2}} \phi(x) g(x) d x \\
& +\int_{A^{c}} \varphi(x) \phi(x) g(x) d x-\int_{A^{c}} \varphi(x) g(x) d x \int_{\mathbb{T}^{2}} \phi(x) g(x) d x \\
= &
\end{aligned}
$$

and

$\operatorname{Cov}\left[1_{A}\left(X^{1}\right) \xi_{1} \psi\left(X^{1}\right), \phi\left(X^{1}\right)\right]=p \int_{A} \psi(x) \phi(x) g(x) d x-p \int_{A} \psi(x) g(x) d x \int_{\mathbb{T}^{2}} \phi(x) g(x) d x=\lambda_{p}$.

Thus according to the central limit theorem

$$
\left(U_{0}^{N}, \varphi\right)+\left(V_{0}^{N}, \psi\right)+\left(Z_{0}^{N}, \phi\right) \rightsquigarrow \mathcal{N}\left(0, \alpha_{p}+\beta_{p}+\sigma^{2}+2\left(\gamma_{p}+\lambda_{p}+\eta_{p}\right)\right) .
$$


Taking $\phi \equiv 0 ; \psi \equiv 0$ and $\varphi \equiv 0$ respectively in (4.7), we obtain

So we deduce that

$$
\begin{aligned}
& \operatorname{Var}\left(\left(U_{0}, \varphi\right)+\left(V_{0}, \psi\right)\right)=\alpha_{p}+\beta_{p}+2 \gamma_{p}, \\
& \operatorname{Var}\left(\left(U_{0}, \varphi\right)+\left(Z_{0}, \phi\right)\right)=\alpha_{p}+\sigma^{2}+2 \eta_{p}, \\
& \operatorname{Var}\left(\left(V_{0}, \psi\right)+\left(Z_{0}, \phi\right)\right)=\beta_{p}+\sigma^{2}+2 \lambda_{p} .
\end{aligned}
$$

$$
\operatorname{Cov}\left(\left(U_{0}, \varphi\right),\left(V_{0}, \psi\right)\right)=\gamma_{p} ; \operatorname{Cov}\left(\left(U_{0}, \varphi\right),\left(Z_{0}, \phi\right)\right)=\eta_{p} \text { and } \operatorname{Cov}\left(\left(V_{0}, \psi\right),\left(Z_{0}, \phi\right)\right)=\lambda_{p} .
$$

Hence from (4.4), (4.5), (4.6), (4.7) and (4.8), we conclude that for any $\varphi, \psi, \phi \in H^{s}$, $\left(\left(U_{0}, \varphi\right),\left(V_{0}, \psi\right),\left(Z_{0}, \phi\right)\right)$ is a Gaussian vector with the same law as the vector given by (4.1), (4.2) and (4.3).

\subsubsection{Conclusion}

The sequence $\left(U_{0}^{N}, V_{0}^{N}, Z_{0}^{N}\right)_{N \geq 1}$ being tight so by Prokhorov's theorem there exists a subsequence still denoted $\left(U_{0}^{N}, V_{0}^{N}, Z_{0}^{N}\right)_{N \geq 1}$ which converge in law in $\left(H^{-s}\left(\mathbb{T}^{2}\right)\right)^{3}$ towards $\left(U_{0}, V_{0}, Z_{0}\right)$. On the other hand $\forall \varphi, \psi, \phi \in H^{s}\left(\left(U_{0}, \varphi\right),\left(V_{0}, \psi\right),\left(Z_{0}, \phi\right)\right) \rightsquigarrow \mathcal{N}\left((0,0,0),\left(\begin{array}{ccc}\alpha_{p} & \gamma_{p} & \eta_{p} \\ \gamma_{p} & \beta_{p} & \lambda_{p} \\ \eta_{p} & \lambda_{p} & \sigma^{2}\end{array}\right)\right)$, hence we conclude that the whole sequence $\left(U_{0}^{N}, V_{0}^{N}, Z_{0}^{N}\right)_{N \geq 1}$ converge in law in $\left(H^{-s}\right)^{3}$ towards $\left(U_{0}, V_{0}, Z_{0}\right)$.

\section{$5 \quad$ Law of Large Numbers}

The aim of this section is to study the convergence of $\left(\mu^{S, N}, \mu^{I, N}\right)$ under Assumption (H1) below, and the convergence of $\mu^{N}$ as $N \rightarrow \infty$.

To this end we are going to:

- Write the system of evolution equations of $\left(\mu^{S, N}, \mu^{I, N}\right)$.

- Study the convergence of $\left\{\mu_{t}^{N}, t \geq 0\right\}$ in $C\left(\mathbb{R}_{+}, \mathcal{M}_{F}\left(\mathbb{T}^{2}\right)\right)$.

- Study the tightness of $\left(\mu^{S, N}, \mu^{I, N}\right)_{N \geq 1}$ in Skorokhod's space $\left(D\left(\mathbb{R}_{+}, \mathcal{M}_{F}\left(\mathbb{T}^{2}\right)\right)\right)^{2}$.

- Show that all limit points $\mu^{S}$ and $\mu^{I}$ of $\left(\mu^{S, N}\right)_{N \geq 1}$ and $\left(\mu^{I, N}\right)_{N \geq 1}$ are absolutely continuous with respect to the Lebesgue measure with density $f_{S}$ and $f_{I}$ bounded by $\delta_{2}\left(\delta_{2}\right.$ is defined in section 3).

- Show that the system of PDEs verified by the pair $\left(f_{S}, f_{I}\right)$ admits a unique solution in $\Lambda=\left\{\left(f_{1}, f_{2}\right) / 0 \leq f_{i} \leq \delta_{2}, i \in\{1,2\}\right\}$.

The following is assumed to hold throughout section 5 .

\section{Assumption (H1): $\quad \mathrm{k}$ is Lipschitz, with the Lipschitz constant $C_{k}$.}

\subsection{System of evolution equations of $\left\{\left(\mu_{t}^{S, N}, \mu_{t}^{I, N}\right), t \geq 0\right\}$}

\subsubsection{Evolution equation of $\left\{\mu_{t}^{S, N}, t \geq 0\right\}$}

Let $\left\{M^{i}, 1 \leq i \leq N\right\}$ be a family of mutualy independant standard (i.e with mean measure the Lebesgue measure ) Poisson Random Measures (in short PRMs) on $\mathbb{R}_{+}^{2}$ which are globally independent of $\left\{X_{t}^{i}, 0 \leq t, 1 \leq i \leq N\right\}$. We note by $\left\{\bar{M}^{i}, 1 \leq i \leq N\right\}$ the family of compensated PRMs. We recall that $\Pi$ is the canonical projection from $\mathbb{R}^{2}$ to $\mathbb{T}^{2}, \widetilde{X}_{t}^{i}=X^{i}+\sqrt{2 \gamma} B_{t}^{i}$ and $X_{t}^{i}=\Pi\left(\widetilde{X}_{t}^{i}\right)$. Now Let $\varphi \in C^{2}\left(\mathbb{T}^{2}\right)$, if we let $\widetilde{\varphi}=\varphi \circ \Pi$, according to the Itô formula, we have

$$
\widetilde{\varphi}\left(\widetilde{X}_{t}^{i}\right)=\widetilde{\varphi}\left(X^{i}\right)+\sqrt{2 \gamma} \int_{0}^{t} \nabla \widetilde{\varphi}\left(\widetilde{X}_{r}^{i}\right) d B_{r}^{i}+\gamma \int_{0}^{t} \triangle \widetilde{\varphi}\left(\widetilde{X}_{r}^{i}\right) d r
$$


hence $\quad \varphi\left(X_{t}^{i}\right)=\varphi\left(X^{i}\right)+\sqrt{2 \gamma} \int_{0}^{t} \nabla \varphi\left(X_{r}^{i}\right) d B_{r}^{i}+\gamma \int_{0}^{t} \triangle \varphi\left(X_{r}^{i}\right) d r$.

Thus $\left\{1_{\left\{E_{t}^{i}=S\right\}} \varphi\left(X_{t}^{i}\right), t \geq 0\right\}$ is a jump process satisfying

$$
\begin{aligned}
1_{\left\{E_{t}^{i}=S\right\}} \varphi\left(X_{t}^{i}\right) & =1_{\left\{E_{0}^{i}=S\right\}} \varphi\left(X^{i}\right)+\sqrt{2 \gamma} \int_{0}^{t} 1_{\left\{E_{r}^{i}=S\right\}} \nabla \varphi\left(X_{r}^{i}\right) d B_{r}^{i}+\gamma \int_{0}^{t} 1_{\left\{E_{r}^{i}=S\right\}} \Delta \varphi\left(X_{r}^{i}\right) d r \\
& -\int_{0}^{t} \int_{0}^{\infty} 1_{\left\{u \leq \beta \sum_{j=1}^{N} \frac{K\left(X_{r}^{i}, X_{r}^{j}\right)}{\sum_{l=1}^{N} K\left(X_{r}^{l}, X_{r}^{j}\right)} 1_{\left\{E_{r}^{j}=I\right\}}\right\}} 1_{\left\{E_{r-}^{i}=S\right\}} \varphi\left(X_{r}^{i}\right) M^{i}(d u, d r) .
\end{aligned}
$$

Taking the sum over $\mathrm{i}$ and multiplying by $\frac{1}{N}$ we obtain

$$
\begin{aligned}
\frac{1}{N} \sum_{i=1}^{N} 1_{\left\{E_{t}^{i}=S\right\}} \varphi\left(X_{t}^{i}\right)= & \frac{1}{N} \sum_{i=1}^{N} 1_{\left\{E_{0}^{i}=S\right\}} \varphi\left(X^{i}\right)+\frac{\sqrt{2 \gamma}}{N} \sum_{i=1}^{N} \int_{0}^{t} 1_{\left\{E_{r}^{i}=S\right\}} \nabla \varphi\left(X_{r}^{i}\right) d B_{r}^{i} \\
& +\frac{\gamma}{N} \sum_{i=1}^{N} \int_{0}^{t} 1_{\left\{E_{r}^{i}=S\right\}} \triangle \varphi\left(X_{r}^{i}\right) d r \\
& -\frac{1}{N} \sum_{i=1}^{N} \int_{0}^{t} \int_{0}^{\infty} 1_{\left\{u \leq \beta \sum_{j=1}^{N} \frac{K\left(X_{r}^{i}, X_{r}^{j}\right)}{\sum_{l=1}^{N} K\left(X_{r}^{l}, X_{r}^{j}\right)}\right.} 1_{\left\{E_{r}^{j}=I\right\}} 1_{\left\{E_{r-}^{i}=S\right\}} \varphi\left(X_{r}^{i}\right) \bar{M}^{i}(d u, d r) \\
& -\frac{1}{N} \sum_{i=1}^{N} \int_{0}^{t} \frac{\beta}{N} \sum_{j=1}^{N} \frac{K\left(X_{r}^{i}, X_{r}^{j}\right)}{\frac{1}{N} \sum_{l=1}^{N} K\left(X_{r}^{l}, X_{r}^{j}\right)} 1_{\left\{E_{r}^{j}=I\right\}} 1_{\left\{E_{r}^{i}=S\right\}} \varphi\left(X_{r}^{i}\right) d r .
\end{aligned}
$$

So

$$
\left(\mu_{t}^{S, N}, \varphi\right)=\left(\mu_{0}^{S, N}, \varphi\right)+\gamma \int_{0}^{t}\left(\mu_{r}^{S, N}, \triangle \varphi\right) d r-\beta \int_{0}^{t}\left(\mu_{r}^{S, N}, \varphi\left(\mu_{r}^{I, N}, \frac{K}{\left(\mu_{r}^{N}, K\right)}\right)\right) d r+M_{t}^{N, \varphi},
$$

where

$$
\left(\mu_{r}^{S, N}, \varphi\left(\mu_{r}^{I, N}, \frac{K}{\left(\mu_{r}^{N}, K\right)}\right)\right)=\int_{\mathbb{T}_{2}} \varphi(x) \int_{\mathbb{T}^{2}} \frac{K(x, y)}{\int_{\mathbb{T}_{2}} K(y, z) \mu_{r}^{N}(d z)} \mu_{r}^{I, N}(d y) \mu_{r}^{S, N}(d x)
$$

and

$$
\begin{aligned}
M_{t}^{N, \varphi}= & -\frac{1}{N} \sum_{i=1}^{N} \int_{0}^{t} \int_{0}^{\infty} 1_{\left\{E_{r^{-}}^{i}=S\right\}} \varphi\left(X_{r}^{i}\right) 1_{\left\{u \leq \beta \sum_{j=1}^{N} \frac{K\left(X_{r}^{i}, X_{r}^{j}\right)}{\left.\sum_{l=1}^{N} K\left(X_{r}^{l}, X_{r}^{j}\right)\right)} 1_{\left\{E_{r}^{j}=I\right\}}\right\}} \bar{M}^{i}(d r, d u) \\
& +\frac{\sqrt{2 \gamma}}{N} \sum_{i=1}^{N} \int_{0}^{t} 1_{\left\{E_{r}^{i}=S\right\}} \nabla \varphi\left(X_{r}^{i}\right) d B_{r}^{i} .
\end{aligned}
$$

\subsubsection{Evolution equation of $\left\{\mu_{t}^{I, N}, t \geq 0\right\}$}

Let $\left\{Q^{i}, 1 \leq i \leq N\right\}$ be a family of mutually independent standard Poisson Random Measures (abreviated below as PRMs) on $\mathbb{R}_{+}^{2}$ which are globally independent of $\left\{X_{t}^{i}, 0 \leq t, 1 \leq i \leq N\right\}$ and $\left\{M^{i}, 1 \leq i \leq N\right\}$. We note by $\left\{\bar{Q}^{i}, 1 \leq i \leq N\right\}$ the family of compensated PRMs.

Let $\varphi \in C^{2}\left(\mathbb{T}^{2}\right),\left\{1_{\left\{E_{t}^{i}=I\right\}} \varphi\left(X_{t}^{i}\right), t \geq 0\right\}$ is a jump process satisfying

$$
\begin{aligned}
1_{\left\{E_{t}^{i}=I\right\}} \varphi\left(X_{t}^{i}\right) & =1_{\left\{E_{0}^{i}=I\right\}} \varphi\left(X^{i}\right)+\sqrt{2 \gamma} \int_{0}^{t} 1_{\left\{E_{r}^{i}=I\right\}} \nabla \varphi\left(X_{r}^{i}\right) d B_{r}^{i}+\gamma \int_{0}^{t} 1_{\left\{E_{r}^{i}=I\right\}} \Delta \varphi\left(X_{r}^{i}\right) d r \\
& +\int_{0}^{t} \int_{0}^{\infty} 1_{\left\{u \leq \beta \sum_{j=1}^{N} \frac{K\left(X_{r}^{i}, X_{r}^{j}\right)}{\sum_{l=1}^{N} K\left(X_{r}^{l}, X_{r}^{j}\right)} 1_{\left\{E_{r}^{j}=I\right\}} 1_{\left\{E_{r-}^{i}=S\right\}} \varphi\left(X_{r}^{i}\right) M^{i}(d u, d r)\right.} \\
& -\int_{0}^{t} \int_{0}^{\alpha} 1_{\left\{E_{r-}^{i}=I\right\}} \varphi\left(X_{r}^{i}\right) Q^{i}(d u, d r) .
\end{aligned}
$$

Summing over i and multiplying by $\frac{1}{N}$ we obtain 


$$
\begin{aligned}
& \frac{1}{N} \sum_{i=1}^{N} 1_{\left\{E_{t}^{i}=I\right\}} \varphi\left(X_{t}^{i}\right)=\frac{1}{N} \sum_{i=1}^{N} 1_{\left\{E_{0}^{i}=I\right\}} \varphi\left(X^{i}\right)+\frac{\sqrt{2 \gamma}}{N} \sum_{i=1}^{N} \int_{0}^{t} 1_{\left\{E_{r}^{i}=I\right\}} \nabla \varphi\left(X_{r}^{i}\right) d B_{r}^{i} \\
& +\frac{\gamma}{N} \sum_{i=1}^{N} \int_{0}^{t} 1_{\left\{E_{r}^{i}=I\right\}} \triangle \varphi\left(X_{r}^{i}\right) d r \\
& +\frac{1}{N} \sum_{i=1}^{N} \int_{0}^{t} \int_{0}^{\infty} 1_{\left\{u \leq \beta \sum_{j=1}^{N} \frac{K\left(X_{r}^{i}, X_{r}^{j}\right)}{\sum_{l=1}^{N} K\left(X_{r}^{l}, X_{r}^{j}\right)} 1_{\left\{E_{r}^{j}=I\right\}}\right.} 1_{\left\{E_{r-}^{i}=S\right\}} \varphi\left(X_{r}^{i}\right) \bar{M}^{i}(d u, d r) \\
& +\frac{1}{N} \sum_{i=1}^{N} \int_{0}^{t} \int_{0}^{\infty} 1_{\left\{u \leq \beta \sum_{j=1}^{N} \frac{K\left(X_{r}^{i}, X_{r}^{j}\right)}{\sum_{l=1}^{N} K\left(X_{r}^{l}, X_{r}^{j}\right)} 1_{\left\{E_{r}^{j}=I\right\}}\right.} 1_{\left\{E_{r}^{i}=S\right\}} \varphi\left(X_{r}^{i}\right) d r d u \\
& -\frac{1}{N} \sum_{i=1}^{N} \int_{0}^{t} \int_{0}^{\alpha} 1_{\left\{E_{r^{-}}^{i}=I\right\}} \varphi\left(X_{r}^{i}\right) \bar{Q}^{i}(d u, d r)-\frac{\alpha}{N} \sum_{i=1}^{N} \int_{0}^{t} 1_{\left\{E_{r}^{i}=I\right\}} \varphi\left(X_{r}^{i}\right) d r .
\end{aligned}
$$

So

$$
\begin{aligned}
\left(\mu_{t}^{I, N} \varphi\right)= & \left(\mu_{0}^{I, N}, \varphi\right)+\gamma \int_{0}^{t}\left(\mu_{r}^{I, N}, \triangle \varphi\right) d r+\beta \int_{0}^{t}\left(\mu_{r}^{S, N}, \varphi\left(\mu_{r}^{I, N}, \frac{K}{\left(\mu_{r}^{N}, K\right)}\right)\right) d r-\alpha \int_{0}^{t}\left(\mu_{r}^{I, N}, \varphi\right) d r \\
& +L_{t}^{N, \varphi}
\end{aligned}
$$

where

$$
\begin{aligned}
L_{t}^{N, \varphi} & \left.=\frac{1}{N} \sum_{i=1}^{N} \int_{0}^{t} \int_{0}^{\infty} 1_{\left\{E_{r-}^{i}=S\right\}} \varphi\left(X_{r}^{i}\right) 1_{\left\{u \leq \beta \sum_{j=1}^{N} \frac{K\left(X_{r}^{i}, X_{r}^{j}\right)}{\sum_{l=1}^{N} K\left(X_{r}^{l}, X_{r}^{j}\right)} 1_{\left\{E_{r}^{j}=I\right\}}\right\}} \bar{M}^{i}(d r, d u)\right) \\
& +\frac{\sqrt{2 \gamma}}{N} \sum_{i=1}^{N} \int_{0}^{t} 1_{\left\{E_{r}^{i}=I\right\}} \nabla \varphi\left(X_{r}^{i}\right) d B_{r}^{i}-\frac{1}{N} \sum_{i=1}^{N} \int_{0}^{t} \int_{0}^{\alpha} 1_{\left\{E_{r^{-}}^{i}=I\right\}} \varphi\left(X_{r}^{i}\right) \bar{Q}^{i}(d r, d u) .
\end{aligned}
$$

\subsection{Convergence of $\left\{\mu_{t}^{N}, t \geq 0\right\}_{N \geq 1}$ in $C\left(\mathbb{R}_{+}, \mathcal{M}_{F}\left(\mathbb{T}^{2}\right)\right)$}

Recall that we equip $\mathcal{M}_{F}\left(\mathbb{T}^{2}\right)$ with the topology of weak convergence and the space of continuous functions from $\mathbb{R}_{+}$to $\mathcal{M}_{F}\left(\mathbb{T}^{2}\right)$, denoted $C\left(\mathbb{R}_{+}, \mathcal{M}_{F}\left(\mathbb{T}^{2}\right)\right)$ with the uniform topology.

It follows from the Itô formula that the processes $\left\{\mu_{t}^{N}, t \geq 0\right\}$ satisfies

$\left(\mu_{t}^{N}, \varphi\right)=\left(\mu_{0}^{N}, \varphi\right)+\gamma \int_{0}^{t}\left(\mu_{r}^{N}, \triangle \varphi\right) d r+\mathcal{H}_{t}^{N, \varphi}$, with $\mathcal{H}_{t}^{N, \varphi}=\frac{\sqrt{2 \gamma}}{N} \sum_{i=1}^{N} \int_{0}^{t} \nabla \varphi\left(X_{r}^{i}\right) d B_{r}^{i}$.

Proposition 5.1. The sequence $\left\{\mu_{t}^{N}, t \geq 0, N \geq 1\right\}$ converges in probability in $C\left(\mathbb{R}_{+}, \mathcal{M}_{F}\left(\mathbb{T}^{2}\right)\right)$ towards $\left\{\mu_{t}, t \geq 0\right\}$, where for each $t \geq 0, \mu_{t}$ is the law of $X_{t}^{1}$ and for any $\varphi \in C^{2}\left(\mathbb{T}^{2}\right), t \geq 0,\left(\mu_{t}, \varphi\right)=\left(\mu_{0}, \varphi\right)+\gamma \int_{0}^{t}\left(\mu_{r}, \triangle \varphi\right) d r$.

Proof. We refer to Theorem 2.2 and Remark page 58 of Roelly [24]. Let $\Pi$ be a dense subset of $C\left(\mathbb{T}^{2}\right)$. In order to prove that $\left(\mu^{N}\right)_{N \geq 1}$ converges in probability in $C\left(\mathbb{R}_{+}, \mathcal{M}_{F}\left(\mathbb{T}^{2}\right)\right)$ towards $\left\{\mu_{t}, t \geq 0\right\}$ it is enough to prove that:

1- $\forall \varphi \in \Pi, \quad\left\{\left(\mu_{t}^{N}, \varphi\right), t \geq 0\right\}_{N \geq 1}$ is tight in $C\left(\mathbb{R}_{+}, \mathbb{R}_{+}\right)$

2- For any $m \geq 1$, any $\left(t_{1}, t_{2} \ldots \ldots ., t_{m}\right) \in \mathbb{R}_{+}^{m}$, and any $\left(\varphi_{1}, \varphi_{2}, \ldots \ldots \ldots, \varphi_{m}\right) \in(\Pi)^{n}$ the sequence $\left(\left(\mu_{t_{1}}^{N}, \varphi_{1}\right), \ldots,\left(\mu_{t_{m}}^{N}, \varphi_{m}\right)\right)$ converges in probability in $\mathbb{R}^{m}$ towards $\left(\left(\mu_{t_{1}}, \varphi_{1}\right), \ldots,\left(\mu_{t_{m}}, \varphi_{m}\right)\right)$

Proof of 1 . We choose $\Pi=C^{2}\left(\mathbb{T}^{2}\right)$. Let $\varphi \in C^{2}\left(\mathbb{T}^{2}\right)$, due to Proposition 37 of Pardoux [22], Corollary page 179 of Billingsleg [6] a sufficient condition for the sequence $\left(\mu^{N}, \varphi\right)_{N \geq 1}$ to be tight in $C\left(\mathbb{R}_{+}, \mathbb{R}_{+}\right)$is that both

- $\left(\mu_{0}^{N}, \varphi\right)$ is tight in $\mathbb{R}$,

- $\forall T>0, \sup _{0 \leq t \leq T}\left(\left|\left(\mu_{t}^{N}, \triangle \varphi\right)\right|+\frac{1}{N}\left(\mu_{t}^{N},(\nabla \varphi)^{2}\right)\right)$ is tight in $\mathbb{R}$.

Since for all $N \geq 0, t \geq 0, \mu_{t}^{N}$ is a probability measure, these two points follow readily from 
the fact that $\varphi, \triangle \varphi$ and $(\nabla \varphi)^{2}$ are bounded on $\mathbb{T}^{2}$.

Proof of 2. According to the law of large numbers, $\forall t \geq 0,\left(\mu_{t}^{N}, \varphi\right) \stackrel{a . s}{\longrightarrow} \mathbb{E}\left(\varphi\left(X_{t}^{1}\right)\right)$ so $\left(\left(\mu_{t_{1}}^{N}, \varphi_{1}\right), . .,\left(\mu_{t_{m}}^{N}, \varphi_{m}\right)\right) \stackrel{a . s}{\longrightarrow}\left(\mathbb{E}\left(\varphi_{1}\left(X_{t}^{1}\right)\right), \ldots, \mathbb{E}\left(\varphi_{m}\left(X_{t}^{1}\right)\right)\right)=\left(\left(\mu_{t_{1}}, \varphi_{1}\right), . .,\left(\mu_{t_{m}}, \varphi_{m}\right)\right)$.

Finally the fact that $\left(\mu_{t}, t \geq 0\right)$ solves the PDE appearing in the statement follows readily from the Itô formula.

Lemma 5.2. For any $t \geq 0$, the measure $\mu_{t}$ is absolutely continuous with respect to the Lebesgue measure and its density $f(t,$.$) verifies \delta_{1} \leq f(t, x) \leq \delta_{2}, \forall x \in \mathbb{T}^{2}$, where $\delta_{1}$ and $\delta_{2}$ are defined in section 3 .

Proof. Given that $\mu_{t}=\mu_{0}+\gamma \int_{0}^{t} \triangle \mu_{r} d r, \mu_{t}=\Upsilon(t) \mu_{0}$.

Thus as from (2.1) in Proposition 2.5, for any measurable subset of $\mathbb{T}^{2}$, with zero Lebesgue measure $\Upsilon(t) 1_{A} \equiv 0$, the absolute continuity of $\mu_{t}$ with respect to the Lebesgue measure follows from the fact that $\mu_{t}=\Upsilon(t) \mu_{0}$. Furthemore we notice that the law $\mu_{t}$ of $X_{t}^{1}$ is absolute continuous with respect to the Lebesgue measure, this being true whether the law $\mu_{0}$ of $X^{1}$ has or not this property.

- Let us now show that $\forall x \in \mathbb{T}^{2}, \delta_{1} \leq f(t, x) \leq \delta_{2}$.

We first recall that $g$ is the density of the law $\mu_{0}$ of $X^{1}$.

Let $P_{t}$ be the heat kernel on the two dimentional torus. As the solution of the heat equation with the initial condition $\phi$, is the function defined on $\mathbb{T}^{2}$ by $\Upsilon(t) \phi(x)=\int_{\mathbb{T}^{2}} P_{t}(x, y) \phi(y) d y$, $\Upsilon(t)$ is non decreasing in the sence that $\forall \varphi, \psi \in L^{2}\left(\mathbb{T}^{2}\right)$ such that $\varphi \leq \psi, \Upsilon(t) \varphi \leq \Upsilon(t) \psi$. So since for any $C \in \mathbb{R}, \Upsilon(t) C=C$ (which follows from (2.1)) and $f(t,)=.\Upsilon(t) g$, the result follows from the facts that $\delta_{1} \leq g \leq \delta_{2}$ and $\Upsilon(t)$ is non decreasing.

\subsection{Tighness and Convergence of $\left(\mu^{S, N}, \mu^{I, N}\right)_{N \geq 1}$ in $\left(D\left(\mathbb{R}_{+}, \mathcal{M}_{F}\left(\mathbb{T}^{2}\right)\right)\right)^{2}$}

Recall that we equip $\mathcal{M}_{F}\left(\mathbb{T}^{2}\right)$ with the topology of weak convergence and the Skorokhod space of càdlàg functions from $\mathbb{R}_{+}$to $\mathcal{M}_{F}\left(\mathbb{T}^{2}\right)$, denoted $D\left(\mathbb{R}_{+}, \mathcal{M}_{F}\left(\mathbb{T}^{2}\right)\right)$ with the Skorokhod topology. We first note that:

$$
\left\{\begin{aligned}
\left(\mu_{t}^{S, N}, 1_{\mathbb{T}^{2}}\right) & =\frac{1}{N} \sum_{i=1}^{N} 1_{\left\{E_{t}^{i}=S\right\}} \leq 1, \\
\left(\mu_{t}^{I, N}, 1_{\mathbb{T}^{2}}\right) & =\frac{1}{N} \sum_{i=1}^{N} 1_{\left\{E_{t}^{i}=I\right\}} \leq 1,
\end{aligned}\right.
$$

and therefore, $\forall \varphi \in C\left(\mathbb{T}^{2}\right)$

$$
\left\{\begin{array}{l}
\left|\left(\mu_{t}^{S, N}, \varphi\right)\right| \leq\|\varphi\|_{\infty} \\
\left|\left(\mu_{t}^{I, N}, \varphi\right)\right| \leq\|\varphi\|_{\infty} .
\end{array}\right.
$$

Lemma 5.3. Let $\mathcal{H}^{1}=\left\{(\mu, \nu, \rho) \in\left(\mathcal{M}\left(\mathbb{T}^{2}\right)\right)^{3} /\left(\nu, 1_{\mathbb{T}^{2}}\right) \leq 1 ;(\mu, \varphi) \leq(\rho, \varphi), \forall \varphi \in C\left(\mathbb{T}^{2} ; \mathbb{R}_{+}\right)\right\}$ For all $(\mu, \nu, \rho) \in \mathcal{H}^{1}, \varphi \in C\left(\mathbb{T}^{2}\right)$, we have

$$
\begin{aligned}
\mid & \left(\mu, \varphi\left(\nu, \frac{K}{(\rho, K)}\right)\right) \mid \leq\|\varphi\|_{\infty} . \\
\text { Proof. }\left|\left(\mu, \varphi\left(\nu, \frac{K}{(\rho, K)}\right)\right)\right| & =\left|\int_{\mathbb{T}^{2}} \varphi(x) \int_{\mathbb{T}^{2}} \frac{K(x, y)}{\int_{\mathbb{W}^{2}} K\left(x^{\prime}, y\right) \rho\left(d x^{\prime}\right)} \nu(d y) \mu(d x)\right| \\
& \leq\|\varphi\|_{\infty}\left|\int_{\mathbb{T}^{2}} \frac{\int_{\mathbb{T}_{2}} K(x, y) \mu(d x)}{\int_{\mathbb{T}^{2}} K\left(x^{\prime}, y\right) \rho\left(d x^{\prime}\right)} \nu(d y)\right| \\
& \leq\|\varphi\|_{\infty},
\end{aligned}
$$

where we have exploited the symetry of $\mathrm{K}: K(x, y)=K(y, x)$ for the first inequality and the facts that $\frac{\int_{\mathbb{\mathbb { V }}^{2}} K(x, y) \mu(d x)}{\int_{\mathbb{T}^{2}} K\left(x^{\prime}, y\right) \rho\left(d x^{\prime}\right)} \leq 1$ and $\left(\nu, 1_{\mathbb{T}^{2}}\right) \leq 1$ for the last inequality. 
We can now establish the wished tightness.

Proposition 5.4. Both sequences $\left(\mu^{S, N}\right)_{N \geq 1}$ and $\left(\mu^{I, N}\right)_{N \geq 1}$ are tight in $D\left(\mathbb{R}_{+}, \mathcal{M}_{F}\left(\mathbb{T}^{2}\right)\right)$.

Proof. - Let us prove that $\left(\mu^{S, N}\right)_{N \geq 1}$ is tight in $D\left(\mathbb{R}_{+}, \mathcal{M}_{F}\left(\mathbb{T}^{2}\right)\right)$.

As already stated in the proof of Proposition 5.1, it suffices to prove that

Let $\varphi \in C^{2}\left(\mathbb{T}^{2}\right)$, we have

$$
\forall \varphi \in C^{2}\left(\mathbb{T}^{2}\right),\left(\left(\mu_{t}^{S, N}, \varphi\right), t \geq 0\right)_{N \geq 1} \text { is tight in } D\left(\mathbb{R}_{+}, \mathbb{R}\right) .
$$

$$
\begin{aligned}
\left(\mu_{t}^{S, N}, \varphi\right) & =\left(\mu_{0}^{S, N}, \varphi\right)+\gamma \int_{0}^{t}\left(\mu_{r}^{S, N}, \triangle \varphi\right) d r-\beta \int_{0}^{t}\left(\mu_{r}^{S, N}, \varphi\left(\mu_{r}^{I, N}, \frac{K}{\left(\mu_{r}^{N}, K\right)}\right)\right) d r+M_{t}^{N, \varphi} \\
& =\left(\mu_{0}^{S, N}, \varphi\right)+\int_{0}^{t} \gamma\left(\mu_{r}^{S, N}, \triangle \varphi\right)-\beta\left(\mu_{r}^{S, N}, \varphi\left(\mu_{r}^{I, N}, \frac{K}{\left(\mu_{r}^{N}, K\right)}\right)\right) d r+M_{t}^{N, \varphi} .
\end{aligned}
$$

We notice that $\left\{\left(\mu_{t}^{S, N}, \varphi\right), t \geq 0\right\}$ is a semi-martingale since $M^{N, \varphi}$ is a square integrable martingale. Indeed, $M^{N, \varphi}$ is a local martingale as the sum of local martingales, and from Lemma 5.3 we deduce that

$$
\begin{aligned}
<M^{N, \varphi}>_{t} & =\frac{\beta}{N} \int_{0}^{t}\left(\mu_{r}^{S, N}, \varphi^{2}\left(\mu_{r}^{I, N}, \frac{K}{\left(\mu_{r}^{N}, K\right)}\right)\right) d r+\frac{2 \gamma}{N} \int_{0}^{t}\left(\mu_{r}^{S, N},(\nabla \varphi)^{2}\right) d r \\
& \leq \frac{\beta\left\|\varphi^{2}\right\|_{\infty} t}{N}+\frac{2 \gamma t}{N}\left\|(\nabla \varphi)^{2}\right\|_{\infty} .
\end{aligned}
$$

Hence $\mathbb{E}\left(\left|M_{t}^{N, \varphi}\right|^{2}\right)=\mathbb{E}\left(<M^{N, \varphi}>_{t}\right)<\infty$.

Consequently

and

$$
\left(\mu_{t}^{S, N}, \varphi\right)=\left(\mu_{0}^{S, N}, \varphi\right)+\int_{0}^{t} \omega_{r}^{N, \varphi} d r+M_{t}^{N, \varphi} \text { with }<M^{N, \varphi}>_{t}=\int_{0}^{t} \varpi_{r}^{N, \varphi} d r
$$

$$
\begin{aligned}
& \omega_{r}^{N, \varphi}=\gamma\left(\mu_{r}^{S, N}, \Delta \varphi\right)-\beta\left(\mu_{r}^{S, N}, \varphi\left(\mu_{r}^{I, N} \frac{K}{\left(\mu_{r}^{N}, K\right)}\right)\right), \\
& \varpi_{r}^{N, \varphi}=\frac{\beta}{N}\left(\mu_{r}^{S, N}, \varphi^{2}\left(\mu_{r}^{I, N}, \frac{K}{\left(\mu_{r}^{N}, K\right)}\right)\right)+\frac{1}{N}\left(\mu_{r}^{S, N},(\nabla \varphi)^{2}\right) .
\end{aligned}
$$

Furthemore $\omega^{N, \varphi}$ and $\varpi^{N, \varphi}$ are progressively measurable since they are adapted and right continuous, so according to Proposition 37 of [22] a sufficient condition for $\left(\left(\mu_{t}^{S, N}, \varphi\right)\right)_{N \geq 1}$ to be tight in $D\left(\mathbb{R}_{+}, \mathbb{R}\right)$ is that both:

- $\left\{\left(\mu_{0}^{S, N}, \varphi\right), N \geq 1\right\}$ is tight in $\mathbb{R}$,

- $\forall T \geq 0, \sup _{0 \leq t \leq T}\left(\left|\omega_{t}^{N, \varphi}\right|+\varpi_{t}^{N, \varphi}\right)$ is tight in $\mathbb{R}$.

These follow readily from the facts that:

$-\left|\left(\mu_{0}^{S, N}, \varphi\right)\right| \leq\|\varphi\|_{\infty}$.

- From Lemma 5.3, $\left|\omega_{t}^{N, \varphi}\right| \leq \gamma\|\triangle \varphi\|_{\infty}+\beta\|\varphi\|_{\infty}$ and $\varpi_{t}^{N, \varphi} \leq \frac{\beta\left\|\varphi^{2}\right\|_{\infty}}{N}+\frac{1}{N}\left\|(\nabla \varphi)^{2}\right\|_{\infty}$.

The same arguments yields the tightness of $\left\{\mu_{t}^{I, N}, t \geq 0, N \geq 1\right\}$ in $D\left(\mathbb{R}_{+}, \mathcal{M}_{F}\left(\mathbb{T}^{2}\right)\right)$.

Proposition 5.5. All limit points $\left(\mu^{S}, \mu^{I}\right)$ of the sequence $\left(\mu^{S, N}, \mu^{I, N}\right)_{N \geq 1}$ are elements of $\left(C\left(\mathbb{R}_{+}, \mathcal{M}_{F}\left(\mathbb{T}^{2}\right)\right)\right)^{2}$.

Proof. Let us prove that $\left\{\mu_{t}^{S}, t \geq 0\right\}$ is continuous. It is enough to prove that $\forall \varphi \in C\left(\mathbb{T}^{2}\right)$, the processes $\left\{\left(\mu_{t}^{S}, \varphi\right), t \geq 0\right\}$ is continuous. However according to Proposition 3.26 page 315 in [12], a sufficient condition for $\left\{\left(\mu_{t}^{S}, \varphi\right), t \geq 0\right\}$ to be continuous is that:

$$
\forall T>0, \forall \varepsilon>0 \lim _{N \rightarrow \infty} \mathbb{P}\left(\sup _{0 \leq t \leq T}\left|\left(\mu_{t}^{S, N}, \varphi\right)-\left(\mu_{t^{-}}^{S, N}, \varphi\right)\right|>\varepsilon\right)=0
$$

Let $\mathrm{T}>0, \varepsilon>0$, since the infection of two individuals can not occur at the same time, we have:

$$
\left|\left(\mu_{t}^{S, N}, \varphi\right)-\left(\mu_{t^{-}}^{S, N}, \varphi\right)\right| \leq \frac{1}{N} \sum_{i=1}^{N}\left|\varphi\left(X_{t}^{i}\right)\right|\left|1_{\left\{E_{t}^{i}=S\right\}}-1_{\left\{E_{t^{-}}^{i}=S\right\}}\right|
$$




$$
\leq \frac{\|\varphi\|_{\infty}}{N} \sum_{i=1}^{N}\left|1_{\left\{E_{t}^{i}=S\right\}}-1_{\left\{E_{t^{-}}^{i}=S\right\}}\right| \leq \frac{\|\varphi\|_{\infty}}{N} .
$$

So for any $\varepsilon>0, \lim _{N \rightarrow \infty} \mathbb{P}\left(\sup _{0 \leq t \leq T}\left|\left(\mu_{t}^{S, N}, \varphi\right)-\left(\mu_{t^{-}}^{S, N}, \varphi\right)\right|>\varepsilon\right)=0$.

By a similar argument we obtain the continuity of $\left\{\mu_{t}^{I}, t \geq 0\right\}$.

Let us now state the main result of this section.

Theorem 5.6. The sequence $\left(\mu^{S, N}, \mu^{I, N}\right)_{N \geq 1}$ converges in probability in $\left(D\left(\mathbb{R}_{+}, \mathcal{M}_{F}\left(\mathbb{T}^{2}\right)\right)\right)^{2}$ to $\left(\mu^{S}, \mu^{I}\right) \in\left(C\left(\mathbb{R}_{+}, \mathcal{M}_{F}\left(\mathbb{T}^{2}\right)\right)\right)^{2}$ where $\forall \varphi \in C^{2}\left(\mathbb{T}^{2}\right),\left\{\left(\left(\mu_{t}^{S}, \varphi\right),\left(\mu_{t}^{I}, \varphi\right)\right), t \geq 0\right\}$ satisfies

$$
\begin{aligned}
& \left(\mu_{t}^{S}, \varphi\right)=\left(\mu_{0}^{S}, \varphi\right)+\gamma \int_{0}^{t}\left(\mu_{r}^{S}, \triangle \varphi\right) d r-\beta \int_{0}^{t}\left(\mu_{r}^{S}, \varphi\left(\mu_{r}^{I}, \frac{K}{\left(\mu_{r}, K\right)}\right)\right) d r \\
& \left(\mu_{t}^{I}, \varphi\right)=\left(\mu_{0}^{I}, \varphi\right)+\gamma \int_{0}^{t}\left(\mu_{r}^{I}, \triangle \varphi\right) d r+\beta \int_{0}^{t}\left(\mu_{r}^{S}, \varphi\left(\mu_{r}^{I}, \frac{K}{\left(\mu_{r}, K\right)}\right)\right) d r-\alpha \int_{0}^{t}\left(\mu_{r}^{I}, \varphi\right) d r
\end{aligned}
$$

\subsubsection{Proof of Theorem 5.6}

By Proposition 5.4, both sequence $\left(\mu^{S, N}\right)_{N \geq 1}$ and $\left(\mu^{I, N}\right)_{N \geq 1}$ are tight in $D\left(\mathbb{R}_{+}, \mathcal{M}_{F}\left(\mathbb{T}^{2}\right)\right)$, so the sequence $\left(\mu^{S, N}, \mu^{I, N}\right)_{N \geq 1}$ is tight in $\left(D\left(\mathbb{R}_{+}, \mathcal{M}_{F}\left(\mathbb{T}^{2}\right)\right)\right)^{2}$. Thus according to Prokhorov's theorem there exists a subsequence of $\left(\mu^{S, N}, \mu^{I, N}\right)_{N \geq 1}$ still denoted $\left(\mu^{S, N}, \mu^{I, N}\right)_{N \geq 1}$ which converges in law towards $\left(\mu^{S}, \mu^{I}\right)$. Hence to complete the proof of Theorm 5.6 it remains to:

- Find the system of PDEs satisfied by $\left\{\left(\mu_{t}^{S}, \mu_{t}^{I}\right), t \geq 0\right\}$.

- Show that $\forall t \geq 0$, the measure $\mu_{t}^{S}$ and $\mu_{t}^{I}$ are absolutely continuous with respect to the Lebesgue measure with densities $f_{S}(t)$ and $f_{I}(t)$ bounded by $\delta_{2}$.

- Show that the system verifies by $\left(f_{S}(t), f_{I}(t)\right)$ admits a unique solution on the set $\Lambda=\left\{\left(f_{1}, f_{2}\right) / 0 \leq f_{i} \leq \delta_{2}, i \in\{1,2\}\right\}$.

We first prove the following Lemmas, which will be useful to establish the system of PDEs satisfied by $\left\{\left(\mu_{t}^{S}, \mu_{t}^{I}\right), t \geq 0\right\}$.

Lemma 5.7. Under the assumption (H1), the function $K$ is Lipschitz on $\mathbb{T}^{2} \times \mathbb{T}^{2}$, with the Lipschitz constant $2 \sqrt{2} C_{k}$.

Proof. Let $x, x^{\prime}, y, y^{\prime} \in \mathbb{T}^{2}$, one has

$$
\begin{aligned}
& \left|K(x, y)-K\left(x^{\prime}, y^{\prime}\right)\right|=\left|k\left(d_{\mathbb{T}^{2}}^{2}(x, y)\right)-k\left(d_{\mathbb{T}^{2}}^{2}\left(x^{\prime}, y^{\prime}\right)\right)\right| \\
& \leq C_{k}\left|d_{\mathbb{T}^{2}}(x, y)-d_{\mathbb{T}^{2}}\left(x^{\prime}, y^{\prime}\right)\right|\left(d_{\mathbb{T}^{2}}(x, y)+d_{\mathbb{T}^{2}}\left(x^{\prime}, y^{\prime}\right)\right) .
\end{aligned}
$$

Furthemore $\left|d_{\mathbb{T}^{2}}(x, y)-d_{\mathbb{T}^{2}}\left(x^{\prime}, y^{\prime}\right)\right| \leq d_{\mathbb{T}^{2}}\left(x, x^{\prime}\right)+d_{\mathbb{T}^{2}}\left(y, y^{\prime}\right)$.

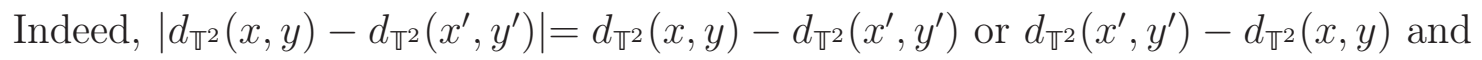

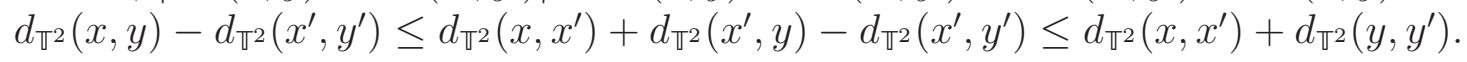

Thus since $\sqrt{2}$ is the maximal distance between two points in $\mathbb{T}^{2}$, we conclude from the above results that

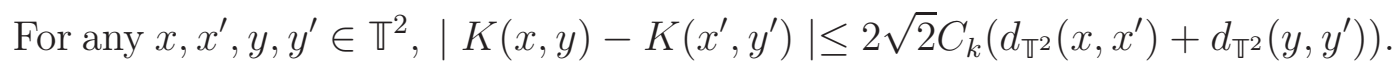

Lemma 5.8. For all $\mu, \nu \in \mathcal{M}\left(\mathbb{T}^{2}\right)$, we have

$$
\sup _{y}\left|\int_{\mathbb{T}^{2}} K\left(x^{\prime}, y\right)\left(\mu\left(d x^{\prime}\right)-\nu\left(d x^{\prime}\right)\right)\right| \leq\left(\|k\|_{\infty}+2 \sqrt{2} C_{k}\right) d_{F}(\mu, \nu) .
$$

Proof. Since from (5.5), for any $y \in \mathbb{T}^{2}$, the function $K(., y)$ is Lipschitz uniformly in $y$ with the Lipschitz constant $2 \sqrt{2} C_{k}$, we have

$$
\begin{aligned}
\left|\int_{\mathbb{T}^{2}} K\left(x^{\prime}, y\right)(\mu-\nu)(d z)\right| & =\left(\|k\|_{\infty}+2 \sqrt{2} C_{k}\right)\left|\int_{\mathbb{T}^{2}} \frac{K\left(x^{\prime}, y\right)}{\left(\|K(., y)\|_{\infty}+2 \sqrt{2} C_{k}\right)}(\mu-\nu)\left(d x^{\prime}\right)\right| \\
& \leq\left(\|k\|_{\infty}+2 \sqrt{2} C_{k}\right) d_{F}(\mu, \nu) .
\end{aligned}
$$

Hence the result. 
Lemma 5.9. The following map is continuous.

$$
\begin{aligned}
G:\left(\mathcal{M}_{F}\left(\mathbb{T}^{2}\right), d_{F}\right) \times\left(\mathcal{M}_{F}\left(\mathbb{T}^{2}\right), d_{F}\right) & \rightarrow\left(\mathcal{M}_{F}\left(\mathbb{T}^{2} \times \mathbb{T}^{2}\right), d_{F}\right) \\
(\mu, \nu) & \longmapsto \mu \otimes \nu
\end{aligned}
$$

where $\forall \phi \in C\left(\mathbb{T}^{2} \times \mathbb{T}^{2}\right),(\mu \otimes \nu, \phi)=\int_{\mathbb{T}^{2} \times \mathbb{T}^{2}} \phi(x, y) \nu(d y) \mu(d x)$

Proof. Let $(\mu, \nu),\left(\mu^{1}, \nu^{1}\right) \in\left(\mathcal{M}_{F}\left(\mathbb{T}^{2}\right)\right)^{2} ; \phi$ a Lipschitz function on $\mathbb{T}^{2} \times \mathbb{T}^{2}$, such that $\|\phi\|_{\infty} \leq 1$ and $\|\phi\|_{L} \leq 1$. We have

$$
\begin{aligned}
\mid \int_{\mathbb{T}^{2} \times \mathbb{T}^{2}} \phi(x, y)(\mu & \left.\otimes \nu-\mu^{1} \otimes \nu^{1}\right)(d x, d y) \mid \leq \\
& \leq \int_{\mathbb{T}^{2}}\left|\int_{\mathbb{T}^{2}} \phi(x, y)\left(\mu-\mu^{1}\right)(d x)\right| \nu(d y)+\int_{\mathbb{T}^{2}}\left|\int_{\mathbb{T}^{2}} \phi(x, y)\left(\nu-\nu^{1}\right)(d y)\right| \mu^{1}(d x) \\
& \leq \nu\left(\mathbb{T}^{2}\right) \sup _{y}\left|\int_{\mathbb{T}^{2}} \phi(x, y)\left(\mu-\mu^{1}\right)(d x)\right|+\mu^{1}\left(\mathbb{T}^{2}\right) \sup _{x}\left|\int_{\mathbb{T}^{2}} \phi(x, y)\left(\nu-\nu^{1}\right)(d y)\right| \\
& \leq C\left(d_{F}\left(\mu, \mu^{1}\right)+d_{F}\left(\nu, \nu^{1}\right)\right),
\end{aligned}
$$

since $\sup _{y}\|\phi(., y)\|_{L} \bigvee \sup _{x}\|\phi(x, .)\|_{L} \leq\|\phi\|_{L} \leq 1$.

We can now establish the system of equations satisfied by $\left(\mu^{S}, \mu^{I}\right)$.

Proposition 5.10. The processes $\left(\mu^{S}, \mu^{I}\right)$ satisfies the equations (5.3) and (5.4)

Proof. We prove this Proposition by taking the limit in the equations (5.1) and (5.2).

1- Let us prove that

One has

$$
\int_{0}^{t}\left(\mu_{r}^{S, N}, \varphi\left(\mu_{r}^{I, N}, \frac{K}{\left(\mu_{r}^{N}, K\right)}\right)\right) d r \stackrel{L}{\rightarrow} \int_{0}^{t}\left(\mu_{r}^{S}, \varphi\left(\mu_{r}^{I}, \frac{K}{\left(\mu_{r}, K\right)}\right)\right) d r .
$$

$$
\begin{aligned}
\left(\mu_{r}^{S, N}, \varphi\left(\mu_{r}^{I, N}, \frac{K}{\left(\mu_{r}^{N}, K\right)}\right)\right) & =\left(\mu_{r}^{I, N}, \frac{\left(\mu_{r}^{S, N}, \varphi K\right)}{\left(\mu_{r}^{N}, K\right)}\right) \\
& =-\left(\mu_{r}^{I, N}, \frac{\left(\mu_{r}^{S, N}, \varphi K\right)}{\left(\mu_{r}^{N}, K\right)\left(\mu_{r}, K\right)}\left(\mu_{r}^{N}-\mu_{r}, K\right)\right)+\left(\mu_{r}^{I, N}, \frac{\left(\mu_{r}^{S, N}, \varphi K\right)}{\left(\mu_{r}, K\right)}\right) .
\end{aligned}
$$

Moreover:

1-1. Since from Lemma $5.2, f(t,$.$) is lower bounded by a positive constant and \forall y \in \mathbb{T}^{2}$, $\int_{\mathbb{T}^{2}} K(x, y) d x$ is a positive constant independent of $\mathrm{y}$,

$$
\exists C>0 \text { such that } \forall y \in \mathbb{T}^{2}, \int_{\mathbb{T}^{2}} K(x, y) f(t, x) d x \geq C .
$$

On the other hand, since from Proposition 5.1 for any $\varphi \in C\left(\mathbb{T}^{2}\right),\left(\mu_{r}^{N}, \varphi\right) \stackrel{P}{\rightarrow}\left(\mu_{r}, \varphi\right)$, $d_{F}\left(\mu_{r}^{N}, \mu_{r}\right) \stackrel{P}{\rightarrow} 0$ (see Lemma 2.7). Thus as $d_{F}\left(\mu_{r}^{N}, \mu_{r}\right) \leq 2$, so from Lemmas 5.3 and 5.8 from the Lebesgue dominated convergence theorem, we have

$$
\begin{gathered}
\mathbb{E}\left(\left|\int_{0}^{t}\left(\mu_{r}^{I, N}, \frac{\left(\mu_{r}^{S, N}, \varphi K\right)}{\left(\mu_{r}^{N}, K\right)\left(\mu_{r}, K\right)}\left(\mu_{r}^{N}-\mu_{r}, K\right)\right) d r\right|\right) \\
\leq \frac{1}{C} \mathbb{E}\left(\int_{0}^{t} \int_{\mathbb{T}^{2}}\left|\frac{\int_{\mathbb{T}^{2}} \varphi(x) K(x, y) \mu_{r}^{S, N}(d x)}{\int_{\mathbb{T}^{2}} K\left(x^{\prime}, y\right) \mu_{r}^{N}\left(d x^{\prime}\right)}\right|\left|\int_{\mathbb{T}^{2}} K\left(x^{\prime}, y\right)\left(\mu_{r}^{N}-\mu_{r}\right)\left(d x^{\prime}\right)\right| \mu_{r}^{I, N}(d y) d r\right) \\
\leq C\|\varphi\|_{\infty}\left(\|k\|_{\infty}+2 \sqrt{2} C_{k}\right) \int_{0}^{t} \mathbb{E}\left(d_{F}\left(\mu_{r}^{N}, \mu_{r}\right)\right) d r \stackrel{N \rightarrow \infty}{\longrightarrow} 0
\end{gathered}
$$

1-2. We have $\left(\mu_{r}^{I, N}, \frac{\left(\mu_{r}^{S, N}, \varphi K\right)}{\left(\mu_{r}, K\right)}\right)=\int_{\mathbb{T}^{2} \times \mathbb{T}^{2}} \frac{\varphi(x) K(x, y)}{\int_{\mathbb{T}^{2}} K\left(x^{\prime}, y\right) \mu_{r}\left(d x^{\prime}\right)} \mu_{r}^{I, N}(d y) \mu_{r}^{S, N}(d x)$.

Moreover since $\int_{\mathbb{T}^{2}} K\left(x^{\prime}, y\right) \mu_{r}\left(d x^{\prime}\right)$ is lower bounded by a positive constant independent of 
$y \in \mathbb{T}^{2}$ and from Lemma 5.7, the map $y \in \mathbb{T}^{2} \mapsto \int_{\mathbb{T}^{2}} K\left(x^{\prime}, y\right) \mu_{r}\left(d x^{\prime}\right)$ is continuous, from Lemma 5.7 again, the map $(x, y) \in \mathbb{T}^{2} \times \mathbb{T}^{2} \mapsto \frac{\varphi(x) K(x, y)}{\int_{\mathbb{T}^{2}} K\left(x^{\prime}, y\right) \mu_{r}\left(d x^{\prime}\right)}$ is continuous and bounded on $\mathbb{T}^{2} \times \mathbb{T}^{2}$. Thus from Lemma 5.9, we deduce that

$$
\int_{\mathbb{T}^{2} \times \mathbb{T}^{2}} \frac{\varphi(x) K(x, y)}{\int_{\mathbb{V}^{2}} K\left(x^{\prime}, y\right) \mu_{r}\left(d x^{\prime}\right)} \mu_{r}^{I, N}(d y) \mu_{r}^{S, N}(d x) \stackrel{L}{\rightarrow} \int_{\mathbb{T}^{2} \times \mathbb{U}^{2}} \frac{\varphi(x) K(x, y)}{\int_{\mathbb{T}^{2}} K\left(x^{\prime}, y\right) \mu_{r}\left(d x^{\prime}\right)} \mu_{r}^{I}(d y) \mu_{r}^{S}(d x) .
$$

2- Since $\triangle \varphi$ and $\varphi$ are continuous and bounded,

$$
\int_{0}^{t}\left(\mu_{r}^{S, N}, \triangle \varphi\right) d r \stackrel{L}{\rightarrow} \int_{0}^{t}\left(\mu_{r}^{S}, \triangle \varphi\right) d r \text { and } \int_{0}^{t}\left(\mu_{r}^{I, N}, \varphi\right) d r \stackrel{L}{\rightarrow} \int_{0}^{t}\left(\mu_{r}^{I}, \varphi\right) d r .
$$

3 - Let us prove that, $M_{t}^{N, \varphi} \stackrel{P}{\rightarrow} 0$ and $L_{t}^{N, \varphi} \stackrel{P}{\rightarrow} 0$.

From Lemma 5.3, we have

$$
\begin{aligned}
\mathbb{E}\left(\left|M_{t}^{N, \varphi}\right|^{2}\right) & =\mathbb{E}\left(<M^{N, \varphi}>_{t}\right) \\
& =\frac{\beta}{N} \int_{0}^{t} \mathbb{E}\left(\left(\mu_{r}^{S, N}, \varphi^{2}\left(\mu_{r}^{I, N}, \frac{K}{\left(\mu_{r}^{N}, K\right)}\right)\right)\right) d r+\frac{1}{N} \int_{0}^{t} \mathrm{E}\left(\left(\mu_{r}^{S, N},(\nabla \varphi)^{2}\right)\right) d r \\
& \leq t \frac{\beta}{N}\left\|\varphi^{2}\right\|_{\infty}+\frac{t}{N}\left\|(\nabla \varphi)^{2}\right\|_{\infty} \stackrel{N \rightarrow \infty}{\longrightarrow} 0 .
\end{aligned}
$$

$M_{t}^{N, \varphi}$ converges to 0 in $L^{2}$, so also in probability. A similar argument yields the fact that $L_{t}^{N, \varphi}$ converges in probability to 0 .

Thus from the results 1-, 2-, 3-, and from the convergence of the initial measures obtained in Theorem 3.1 we conclude that $\left(\mu^{S}, \mu^{I}\right)$ satisfies the equations (5.3) and (5.4).

Proposition 5.11. $\forall t \geq 0$, the measures $\mu_{t}^{S}$ and $\mu_{t}^{I}$ are absolutely continuous with respect to the Lebesgue measure and their densities $f_{S}(t,$.$) and f_{I}(t,$.$) are bounded by \delta_{2}$.

Proof. For any $t \geq 0$, we have $\mu_{t}^{R, N}=\frac{1}{N} \sum_{i=1}^{N} 1_{\left\{E_{t}^{i}=R\right\}} \delta_{X_{t}^{i}}=\mu_{t}^{N}-\mu_{t}^{S, N}-\mu_{t}^{I, N}$.

Furthemore:

- $\mu_{t}^{S, N}+\mu_{t}^{I, N}$ weakly converges towards $\mu_{t}^{S}+\mu_{t}^{I}$ since $\left(\mu_{t}^{S, N}, \mu_{t}^{I, N}\right)$ weakly converges towards $\left(\mu_{t}^{S}, \mu_{t}^{I}\right)$ and the map $(\mu, \nu) \in\left(\mathcal{M}_{F}\left(\mathbb{T}_{2}\right), d_{F}\right)^{2} \mapsto \mu+\nu$ is continuous,

- $\mu_{t}^{N}$ converges in probability towards the measure $\mu_{t}$ which is deterministic $\left(\mu_{t}=\Upsilon(t) \nu\right)$, thus $\mu_{t}^{R, N}=\mu_{t}^{N}-\mu_{t}^{S, N}-\mu_{t}^{I, N}$ weakly converges towards $\mu_{t}-\mu_{t}^{S}-\mu_{t}^{I}$.

Hence as the measures $\mu_{t}^{S}, \mu_{t}^{I}$ and $\mu_{t}-\mu_{t}^{S}-\mu_{t}^{I}$ are non negative (since $\mu_{t}^{S, N}, \mu_{t}^{I, N}$ and $\mu_{t}^{R, N}$ are non negative), we can conclude that

- $\mu_{t}^{S}$ and $\mu_{t}^{I}$ are absolutely continuous with respect to the Lebesgue measure since $\mu_{t}$ has

this property;

- their densities satisfy $f_{S}(t,)+.f_{I}(t,.) \leq f(t,.) \leq \delta_{2}$.

Proposition 5.12. The pair of densities $\left(f_{S}(t,),. f_{I}(t,).\right)$ of the pair of measures $\left(\mu_{t}^{S}, \mu_{t}^{I}\right)$ satisfies

$$
\begin{aligned}
& f_{S}(t)=\Upsilon(t) f_{S}(0)-\beta \int_{0}^{t} \Upsilon(t-r)\left[f_{S}(r) \int_{\mathbb{T}^{2}} \frac{K(., y)}{\int_{\mathbb{T}^{2}} K\left(x^{\prime}, y\right) f\left(r, x^{\prime}\right) d x^{\prime}} f_{I}(r, y) d y\right] d r, \\
& f_{I}(t)=\Upsilon(t) f_{I}(0)+\beta \int_{0}^{t} \Upsilon(t-r)\left[f_{S}(r) \int_{\mathbb{T}^{2}} \frac{K(., y)}{\int_{\mathbb{U}^{2}} K\left(x^{\prime}, y\right) f\left(r, x^{\prime}\right) d x^{\prime}} f_{I}(r, y) d y\right] d r \\
& -\alpha \int_{0}^{t} \Upsilon(t-r) f_{I}(r) d r
\end{aligned}
$$

Moreover the system formed by the equations (5.6) and (5.7) admits a unique solution on the set $\Lambda=\left\{\left(f_{1}, f_{2}\right) / 0 \leq f_{i} \leq \delta_{2}, i \in\{1,2\}\right\}$. 
Proof. Recall that $\forall y \in \mathbb{T}^{2},\left(\mu_{r}, K(., y)\right)=\int_{\mathbb{T}^{2}} K\left(x^{\prime}, y\right) f\left(r, x^{\prime}\right) d x^{\prime} \geq C$. From the equations (5.3) and (5.4), it is easy to deduce that $\left(f_{S}(t,),. f_{I}(t,).\right)$ satisfies the equations (5.6) and (5.7). Let $\left(f_{S}^{1}(t), f_{I}^{1}(t)\right),\left(f_{S}^{2}(t), f_{I}^{2}(t)\right) \in \Lambda$ be two solutions of the system formed by equations (5.6) and (5.7) with the same initial value. Noticing that for any $\varphi \in L^{2}\left(\mathbb{T}^{2}\right),\|\Upsilon(t) \varphi\|_{\infty} \leq\|\varphi\|_{\infty}$ (see Lemma 2.6) and $\left\|\int_{\mathbb{T}^{2}} K(\cdot, y) \varphi(y) d y\right\|_{\infty} \leq C\|\varphi\|_{\infty}$, we have

$$
\begin{aligned}
\| f_{S}^{2}(t)-f_{S}^{1}(t) & \left\|_{\infty} \leq \beta \int_{0}^{t}\right\| \Upsilon(t-r)\left[\left(f_{S}^{2}(r)-f_{S}^{1}(r)\right) \int_{\mathbb{J}^{2}} \frac{K(., y)}{\int_{\mathbb{T}^{2}} K\left(x^{\prime}, y\right) f\left(r, x^{\prime}\right) d x^{\prime}} f_{I}^{1}(r, y) d y\right] \|_{\infty} d r \\
& +\beta \int_{0}^{t}\left\|\Upsilon(t-r)\left[f_{S}^{2}(t) \int_{\mathbb{T}^{2}} \frac{K(., y)}{\int_{\mathbb{T}^{2}} K\left(x^{\prime}, y\right) f\left(r, x^{\prime}\right) d x^{\prime}}\left(f_{I}^{2}(r, y)-f_{I}^{1}(r, y)\right) d y\right]\right\|_{\infty} d r \\
\leq & \frac{\beta}{C} \int_{0}^{t}\left\|f_{S}^{2}(r)-f_{S}^{1}(r)\right\|_{\infty}\left\|\int_{\mathbb{T}^{2}} K(., y) f_{I}^{1}(r, y) d y\right\|_{\infty} d r \\
& +\frac{\beta}{C} \int_{0}^{t}\left\|f_{S}^{2}(r)\right\|_{\infty}\left\|\int_{\mathbb{T}^{2}} K(., y)\left(f_{I}^{2}(r, y)-f_{I}^{1}(r, y)\right) d y\right\|_{\infty} d r \\
\leq & \beta C \delta_{2} \int_{0}^{t}\left\{\left\|f_{S}^{2}(r)-f_{S}^{1}(r)\right\|_{\infty}+\left\|f_{I}^{2}(r)-f_{I}^{1}(r)\right\|_{\infty}\right\} d r .
\end{aligned}
$$

Furthemore as $f_{I}^{2}(t)-f_{I}^{1}(t)=-\left(f_{S}^{2}(t)-f_{S}^{1}(t)\right)-\alpha \int_{0}^{t} \Upsilon(t-r)\left(f_{I}^{2}(r)-f_{I}^{1}(r)\right) d r$,

then $\left\|f_{I}^{2}(t)-f_{I}^{1}(t)\right\|_{\infty} \leq\left\|f_{S}^{2}(t)-f_{S}^{1}(t)\right\|_{\infty}+\alpha \int_{0}^{t}\left\|f_{I}^{2}(r)-f_{I}^{1}(r)\right\|_{\infty} d r$.

Hence summing (5.8) and (5.9) and applying Gronwall's lemma, we obtain $f_{s}^{1}(t)=f_{s}^{2}(t)$ and $f_{I}^{1}(t)=f_{I}^{2}(t)$.

We can now finish the proof of Theorem 5.6.

Since $\left(\mu^{S, N}, \mu^{I, N}\right)_{N \geq 1}$ is tight in $\left(D\left(\mathbb{R}_{+}, \mathcal{M}_{F}\left(\mathbb{T}^{2}\right)\right)\right)^{2}$, and all converging subsequences of the sequence $\left(\mu^{S, N}, \mu^{I, N}\right)_{N \geq 1}$ weakly converge to the same limit $\left(\mu^{S}, \mu^{I}\right)$, the sequence $\left(\mu^{S, N}, \mu^{I, N}\right)_{N \geq 1}$ weakly converge in $\left(D\left(\mathbb{R}_{+}, \mathcal{M}_{F}\left(\mathbb{T}^{2}\right)\right)\right)^{2}$ towards $\left(\mu^{S}, \mu^{I}\right)$; furthemore $\left(\mu^{S}, \mu^{I}\right)$ is deterministic, so we have convergence in probability.

\section{Central Limit Theorem}

In this section we will study the convergence of $\left(U^{N}=\sqrt{N}\left(\mu^{S, N}-\mu^{S}\right), V^{N}=\sqrt{N}\left(\mu^{I, N}-\mu^{I}\right)\right)$ under the assuption (H2) below and the convergence of $Z^{N}=\sqrt{N}\left(\mu^{N}-\mu\right)$ as $N \rightarrow \infty$.

Note that the trajectories of these processes belong to $\left(D\left(\mathbb{R}_{+}, \mathcal{E}\left(\mathbb{T}^{2}\right)\right)\right)^{2}$ and $C\left(\mathbb{R}_{+}, \mathcal{E}\left(\mathbb{T}^{2}\right)\right)$ respectively, where $\mathcal{E}\left(\mathbb{T}^{2}\right)$ is the space of signed measures on the torus, which can be seen as the dual of $C\left(\mathbb{T}^{2}\right)$. However, since the limit processes may be less regular than their approximations we will first:

- Establish the equations verified by the process $Z^{N}$ and by the pair $\left(U^{N}, V^{N}\right)$.

- Fix the space in which the convergence results will be established.

Then we will study the convergence of the above sequences.

The following is assumed to hold throughout section 6 .

Assumption (H2): $k \in C^{3}\left(\mathbb{R}_{+}\right)$.

Remark 6.1. Let $x \in \mathbb{T}^{2}$, if we let $A(x)=\operatorname{support}\{K(x,)$.$\} , from (1.1) and under (H2), we$ have

$-\forall \eta \in \mathbb{N}^{2},|\eta| \leq 2$ the map $y \in A(x) \mapsto D^{\eta} K(x,$.$) is Lipschitz and bounded with the Lipschitz$ constant independent of $x$.

$-\forall \eta \in \mathbb{N}^{2},|\eta| \leq 3$ the map $y \in A(x) \mapsto D^{\eta} K(x,$.$) is continuous and bounded by C \max _{0 \leq|\eta| \leq 3}\left\|k^{(|\eta|)}\right\|_{\infty}$. 
Indeep, this two points follow from the facts that:

- If $\mathcal{C}_{1}=\left\{2 k^{\prime}\left(\|x-y\|^{2}\right), 4 k^{\prime \prime}\left(\|x-y\|^{2}\right), 8 k^{(3)}\left(\|x-y\|^{2}\right)\right\}$ and

$\mathcal{C}_{2}=\left\{\left(x_{1}-y_{1}\right)^{n}\left(x_{2}-y_{2}\right)^{m},(n, m) \in\{0,1,2,3\}^{2}\right\}, \forall|\eta| \leq 3, D^{\eta} K(x, y)=D^{\eta} k\left(\|x-y\|^{2}\right)$ is written as the sum of the products of elements of $\mathcal{C}_{1}$ and $\mathcal{C}_{2}$;

- $\forall|\eta| \leq 2,\left|k^{(|\eta|)}\right|$ is locally Lipschitz in $\mathbb{R}_{+}$and $\forall|\eta| \leq 3,\left|k^{(|\eta|)}\right|$ is bounded in $\mathbb{R}_{+}$;

$-\sqrt{2}$ is the maximal distance between two points of the torus.

Lemma 6.2. Under the assumption (H2), we have $\sup _{x}\|K(x, .)\|_{H^{3}}<\infty$.

Proof. From (1.1), if we let $\forall x \in \mathbb{T}^{2}, A(x)=\operatorname{support}\{K(x,)$.$\} , we will have$

$$
\begin{aligned}
\|K(x, \cdot)\|_{H^{3}} & =\sum_{|\eta| \leq 3} \int_{A(x)}\left|D^{\eta} k\left(\|y-x\|^{2}\right)\right|^{2} d y \\
& \leq C
\end{aligned}
$$

where the first inequality follows from Remark 6.1.

\subsection{Evolution equations of $Z^{N}$ and of the pair $\left(U^{N}, V^{N}\right)$}

\subsubsection{Evolution equation of $Z^{N}=\sqrt{N}\left(\mu^{N}-\mu\right)$}

Let $\varphi \in C^{2}\left(\mathbb{T}^{2}\right)$, we have

$\left(\mu_{t}^{N}, \varphi\right)=\left(\mu_{0}^{N}, \varphi\right)+\gamma \int_{0}^{t}\left(\mu_{r}^{N}, \triangle \varphi\right) d r+\mathcal{H}_{t}^{N, \varphi}$, where $\mathcal{H}_{t}^{N, \varphi}=\frac{\sqrt{2 \gamma}}{N} \sum_{i=1}^{N} \int_{0}^{t} \nabla \varphi\left(X_{r}^{i}\right) d X_{r}^{i}$

$\left(\mu_{t}, \varphi\right)=\left(\mu_{0}, \varphi\right)+\gamma \int_{0}^{t}\left(\mu_{r}, \triangle \varphi\right) d r$

hence $\left(Z_{t}^{N}, \varphi\right)=\left(Z_{0}^{N}, \varphi\right)+\gamma \int_{0}^{t}\left(Z_{r}^{N}, \triangle \varphi\right) d r+\widetilde{\mathcal{H}}_{t}^{N, \varphi}$, where $\widetilde{\mathcal{H}}_{t}^{N, \varphi}=\sqrt{N} \mathcal{H}_{t}^{N, \varphi}$.

\subsubsection{System of evolution equations of the pair $\left(U^{N}, V^{N}\right)$}

Let $\varphi \in C^{2}\left(\mathbb{T}^{2}\right)$, we have

$$
\begin{aligned}
& \left(\mu_{t}^{S, N}, \varphi\right)=\left(\mu_{0}^{S, N}, \varphi\right)+\gamma \int_{0}^{t}\left(\mu_{r}^{S, N}, \triangle \varphi\right) d r-\beta \int_{0}^{t}\left(\mu_{r}^{S, N}, \varphi\left(\mu_{r}^{I, N}, \frac{K}{\left(\mu_{r}^{N}, K\right)}\right)\right) d r+M_{t}^{N, \varphi}, \\
& \left(\mu_{t}^{S}, \varphi\right)=\left(\mu_{0}^{S}, \varphi\right)+\gamma \int_{0}^{t}\left(\mu_{r}^{S}, \triangle \varphi\right) d r-\beta \int_{0}^{t}\left(\mu_{r}^{S}, \varphi\left(\mu_{r}^{I}, \frac{K}{\left(\mu_{r}, K\right)}\right)\right) d r .
\end{aligned}
$$

Note first that $\left(\mu_{r}^{S, N}, \varphi\left(\mu_{r}^{I, N}, \frac{K}{\left(\mu_{r}^{N}, K\right)}\right)\right)=\int_{\mathbb{T}^{2}} \varphi(x) \int_{\mathbb{T}^{2}} \frac{K(x, y)}{\int_{\mathbb{T}^{2}} K\left(x^{\prime}, y\right) \mu^{N}\left(d x^{\prime}\right)} \mu_{r}^{I, N}(d y) \mu_{r}^{S, N}(d x)$

Thus

$$
\begin{aligned}
& =\int_{\mathbb{T}^{2}} \frac{\int_{\mathbb{T}^{2}} \varphi(x) K(x, y) \mu_{r}^{S, N}(d x)}{\int_{\mathbb{T}^{2}} K\left(x^{\prime}, y\right) \mu^{N}\left(d x^{\prime}\right)} \mu_{r}^{I, N}(d y) \\
& =\left(\mu_{r}^{I, N}, \frac{\left(\mu_{r}^{S, N}, \varphi K\right)}{\left(\mu_{r}^{N}, K\right)}\right) .
\end{aligned}
$$

$$
\begin{aligned}
\left(U_{t}^{N}, \varphi\right)= & \left(U_{0}^{N}, \varphi\right)+\gamma \int_{0}^{t}\left(U_{r}^{N}, \triangle \varphi\right) d r-\beta \int_{0}^{t}\left(\sqrt{N} \mu_{r}^{I, N}, \frac{\left(\mu_{r}^{S, N}, \varphi K\right)}{\left(\mu_{r}^{N}, K\right)}\right) d r \\
& +\beta \int_{0}^{t}\left(\sqrt{N} \mu_{r}^{I}, \frac{\left(\mu_{r}^{S}, \varphi K\right)}{\left(\mu_{r}, K\right)}\right) d r+\sqrt{N} M_{t}^{N, \varphi} \\
= & \left(U_{0}^{N}, \varphi\right)+\gamma \int_{0}^{t}\left(U_{r}^{N}, \triangle \varphi\right) d r+\beta \int_{0}^{t}\left(\mu_{r}^{I, N}, \frac{\left(\mu_{r}^{S, N}, \varphi K\right)}{\left(\mu_{r}^{N}, K\right)\left(\mu_{r}, K\right)}\left(Z_{r}^{N}, K\right)\right) d r \\
- & \beta \int_{0}^{t}\left(\mu_{r}^{I, N}, \frac{\left(U_{r}^{N}, \varphi K\right)}{\left(\mu_{r}, K\right)}\right) d r-\beta \int_{0}^{t}\left(V_{r}^{N}, \frac{\left(\mu_{r}^{S}, \varphi K\right)}{\left(\mu_{r}, K\right)}\right) d r+\sqrt{N} M_{t}^{N, \varphi} .
\end{aligned}
$$


Hence if we let $\widetilde{M}_{t}^{N, \varphi}=\sqrt{N} M_{t}^{N, \varphi}$, one has

$$
\begin{aligned}
\left(U_{t}^{N}, \varphi\right)= & \left(U_{0}^{N}, \varphi\right)+\gamma \int_{0}^{t}\left(U_{r}^{N}, \triangle \varphi\right) d r+\beta \int_{0}^{t}\left(Z_{r}^{N}, G_{r}^{S, I, N} \varphi\right) d r-\beta \int_{0}^{t}\left(U_{r}^{N}, G_{r}^{I, N} \varphi\right) d r \\
& -\beta \int_{0}^{t}\left(V_{r}^{N}, G_{r}^{S} \varphi\right) d r+\widetilde{M}_{t}^{N, \varphi}
\end{aligned}
$$

and also

$$
\begin{aligned}
\left(V_{t}^{N}, \varphi\right)= & \left(V_{0}^{N}, \varphi\right)+\gamma \int_{0}^{t}\left(V_{r}^{N}, \triangle \varphi\right) d r-\beta \int_{0}^{t}\left(Z_{r}^{N}, G_{r}^{S, I, N} \varphi\right) d r+\beta \int_{0}^{t}\left(U_{r}^{N}, G_{r}^{I, N} \varphi\right) d r \\
& +\beta \int_{0}^{t}\left(V_{r}^{N}, G_{r}^{S} \varphi\right) d r-\alpha \int_{0}^{t}\left(V_{r}^{N}, \varphi\right) d r+\widetilde{L}_{t}^{N, \varphi},
\end{aligned}
$$

where $\forall x, y, x^{\prime} \in \mathbb{T}^{2}$,

$$
\begin{aligned}
& G_{r}^{S, I, N} \varphi\left(x^{\prime}\right)=\left(\mu_{r}^{I, N}, K\left(x^{\prime}, .\right) \frac{\left(\mu_{r}^{S, N}, \varphi K\right)}{\left(\mu_{r}^{N}, K\right)\left(\mu_{r}, K\right)}\right) \\
&=\int_{\mathbb{T}^{2}} K\left(x^{\prime}, y\right) \frac{\int_{\mathbb{T}^{2}} \varphi(x) K(x, y) \mu_{r}^{S, N}(d x)}{\int_{\mathbb{T}^{2}} K\left(y^{\prime}, y\right) \mu_{r}^{N}\left(d y^{\prime}\right) \int_{\mathbb{T}^{2}} K\left(y^{\prime}, y\right) \mu_{r}\left(d y^{\prime}\right)} \mu_{r}^{I, N}(d y), \\
& G_{r}^{I, N} \varphi(x)= \varphi(x)\left(\mu_{r}^{I, N}, \frac{K(x, .)}{\left(\mu_{r}, K\right)}\right)=\varphi(x) \int_{\mathbb{T}^{2}} \frac{K(x, y)}{\int_{\mathbb{T}^{2}} K\left(y^{\prime}, y\right) \mu_{r}\left(d y^{\prime}\right)} \mu_{r}^{I, N}(d y), \\
& G_{r}^{S} \varphi(y)=\frac{\left(\mu_{r}^{S}, \varphi K(., y)\right)}{\left(\mu_{r}, K(., y)\right)}=\frac{\int_{\mathbb{T}^{2}} \varphi(x) K(x, y) \mu_{r}^{S}(d x)}{\int_{\mathbb{T}^{2}} K\left(y^{\prime}, y\right) \mu_{r}\left(d y^{\prime}\right)}
\end{aligned}
$$

\subsection{The space of convergence of the sequences $Z^{N}$ and $\left(U^{N}, V^{N}\right)$}

We first recall that for any $s>0$, the family $\left(\rho_{n_{1}, n_{2}}^{i, s}\right)_{i, n_{1}, n_{2}}$ (as defined in Proposition 2.2) is an orthonormal basis of $H^{s}\left(\mathbb{T}^{2}\right)$.

Proposition 6.3. Every limit point $W^{1}$ of the seguence $\left(\widetilde{M}^{N}\right)_{N \geq 1}$ satisfies

$$
\forall t \geq 0, \mathbb{E}\left(\left\|W_{t}^{1}\right\|_{H^{-s}}^{2}\right)<\infty \quad \text { iff } s>2 .
$$

Proof. We have

$$
\begin{aligned}
\widetilde{M}_{t}^{N, \varphi}=- & \frac{1}{\sqrt{N}} \sum_{i=1}^{N} \int_{0}^{t} \int_{0}^{\infty} 1_{\left\{E_{r-}^{i}=S\right\}} \varphi\left(X_{r}^{i}\right) 1_{\left\{u \leq \frac{\beta}{N} \sum_{j=1}^{N} \frac{K\left(X_{r}^{i}, X_{r}^{j}\right)}{\left(\mu_{r}^{N}, K\left(, X_{r}^{i}\right)\right)} 1_{\left\{E_{r}^{j}=I\right\}}\right\}} \bar{M}^{i}(d r, d u) \\
& +\sqrt{\frac{2 \gamma_{S}}{N} \sum_{i=1}^{N} \int_{0}^{t} 1_{\left\{E_{r}^{i}=S\right\}} \nabla \varphi\left(X_{r}^{i}\right) d B_{r}^{i} .} \\
<\widetilde{M}^{N, \varphi}>_{t} & =\beta \int_{0}^{t}\left(\mu_{r}^{S, N}, \varphi^{2}\left(\mu_{r}^{I, N}, \frac{K}{\left(\mu_{r}^{N}, K\right)}\right)\right) d r+2 \gamma \int_{0}^{t}\left(\mu_{r}^{S, N},(\nabla \varphi)^{2}\right) d r
\end{aligned}
$$

and it follows from Theorem 5.6 that

$$
<\widetilde{M}^{N, \varphi}>_{t} \stackrel{P}{\rightarrow} \int_{0}^{t}\left\{\beta\left(\mu_{r}^{S}, \varphi^{2}\left(\mu_{r}^{I}, \frac{K}{\left(\mu_{r}, K\right)}\right)\right)+2 \gamma\left(\mu_{r}^{S},(\nabla \varphi)^{2}\right)\right\} d r
$$

furthemore $\int_{0}^{t}\left\{\beta\left(\mu_{r}^{S}, \varphi^{2}\left(\mu_{r}^{I}, \frac{K}{\left(\mu_{r}, K\right)}\right)\right)+2 \gamma\left(\mu_{r}^{S},(\nabla \varphi)^{2}\right)\right\} d r$ being the quadratic variation of a Gaussian martingale of the form $\left(W^{1}, \varphi\right)$, our aim is to find the smallest value of $\mathrm{s}$ for which $\mathbb{E}\left(\left\|W_{t}^{1}\right\|_{H^{-s}}^{2}\right)<\infty$. We have

$\mathbb{E}\left(\left\|W_{t}^{1}\right\|_{H^{-s}}^{2}\right)=\mathbb{E}\left(\sum_{i, n_{1}, n_{2}}\left|\left(W_{t}^{1}, \rho_{n_{1}, n_{2}}^{i, s}\right)\right|^{2}\right)=\sum_{i, n_{1}, n_{2}} \mathbb{E}\left(<\left(W^{1}, \rho_{n_{1}, n_{2}}^{i, s}\right)>_{t}\right)$.

However as $\int_{\mathbb{T}^{2}} \frac{\int_{\mathbb{\mathbb { N }}^{2}} K(x, y) \mu_{r}^{S}(d x)}{\int_{\mathbb{T}^{2}} K\left(x^{\prime}, y\right) \mu_{r}\left(d x^{\prime}\right)} \mu_{r}^{I}(d y) \leq 1$, then from Lemma 8.1 in the Appendix below, 
iff $\mathrm{s}>2$, we will have

$$
\begin{aligned}
\sum_{i, n_{1}, n_{2}}<W^{1}, \rho_{n_{1}, n_{2}}^{i}> & =\sum_{i, n_{1}, n_{2}} \int_{0}^{t} \beta\left(\mu_{r}^{S},\left(\rho_{n_{1}, n_{2}}^{i, s}\right)^{2}\left(\mu_{r}^{I}, \frac{K}{\left(\mu_{r}, K\right)}\right)\right)+2 \gamma\left(\mu_{r}^{S},\left(\nabla \rho_{n_{1}, n_{2}}^{i, s}\right)^{2}\right) d r \\
& \leq \int_{0}^{t}\left\{\beta \int_{\mathbb{T}^{2}} \int_{\mathbb{T}^{2}} \sum_{i, n_{1}, n_{2}}\left(\rho_{n_{1}, n_{2}}^{i, s}\right)^{2}(x) \frac{K(x, y)}{\int_{\mathbb{T}^{2}} K\left(y, x^{\prime}\right) \mu_{r}\left(d x^{\prime}\right)} \mu_{r}^{I}(d y) \mu_{r}^{S}(d x)\right. \\
& \left.+2 \gamma \int_{\mathbb{T}^{2}} \sum_{i, n_{1}, n_{2}}\left(\nabla \rho_{n_{1}, n_{2}}^{i, s}(x)\right)^{2} \mu_{r}^{S}(d x)\right\} d r \\
& \leq \beta C \int_{0}^{t} \int_{\mathbb{T}^{2}} \frac{\int_{\mathbb{T}^{2}} K(x, y) \mu_{r}^{S}(d x)}{\int_{\mathbb{T}^{2}} K\left(y, x^{\prime}\right) \mu_{r}\left(d x^{\prime}\right)} \mu_{r}^{I}(d y) d r+2 \gamma C \int_{0}^{t} \int_{\mathbb{T}^{2}} \mu_{r}^{S}(d x) d r \\
& \leq C t(\beta+2 \gamma) .
\end{aligned}
$$

The result follows. result.

By Doob's inequality and by calculations similar to those done above we obtain the following

Corollary 6.4. $\forall T>0, s>2, \exists C_{1}(T)>0, C_{2}(T)>0, C_{3}(T)>0$ such that:

$$
\begin{aligned}
& \sup _{N \geq 1} \mathbb{E}\left(\sup _{0 \leq t \leq T}\left\|\widetilde{\mathcal{H}}_{t}^{N}\right\|_{H^{-s}}^{2}\right) \leq C_{1}(T), \\
& \sup \mathbb{E}\left(\sup _{N \geq 1}\left\|\widetilde{M}_{t}^{N}\right\|_{H^{-s}}^{2}\right) \leq C_{2}(T), \\
& \sup _{N \geq 1} \mathbb{E}\left(\sup _{0 \leq t \leq T}\left\|\widetilde{L}_{t}^{N}\right\|_{H^{-s}}^{2}\right) \leq C_{3}(T) .
\end{aligned}
$$

In the rest of this section we arbitrarily choose $2<\mathrm{s}<3$, and we prove that the sequences $\left(Z^{N}\right)_{N \geq 1}$ and $\left(U^{N}, V^{N}\right)_{N \geq 1}$ converge in law in $C\left(\mathbb{R}_{+}, H^{-s}\right)$ and in $\left(D\left(\mathbb{R}_{+}, H^{-s}\right)\right)^{2}$ respectively, where we have equipped $C\left(\mathbb{R}_{+}, H^{-s}\right)$ with the uniform topology and $D\left(\mathbb{R}_{+}, H^{-s}\right)$ with the Skorokhod topology.

\subsection{Tighness and Convergence of $\left(Z^{N}\right)_{N \geq 1}$}

Recall that the sequence $\left(Z^{N}\right)_{N \geq 1}$ satisfies (6.1). We first give an estimate for the norm of the fluctuations process $Z^{N}$ which is not uniform in $N$.

Lemma 6.5. For all $N \geq 1, Z^{N} \in C\left(\mathbb{R}_{+}, H^{-s}\right)$.

Proof. Since s $>2, H^{s}\left(\mathbb{T}_{2}\right) \hookrightarrow C\left(\mathbb{T}_{2}\right)$ (see Proposition 2.4). Thus

$$
\begin{aligned}
\left|\left(Z_{t}^{N}, \varphi\right)\right| & =\sqrt{N}\left|\frac{1}{N} \sum_{i=1}^{N} \varphi\left(X_{t}^{i}\right)-\left(\mu_{t}, \varphi\right)\right| \\
& \leq \sqrt{N}\left(\frac{1}{N}\left(\sum_{i=1}^{N}\left|\varphi\left(X_{t}^{i}\right)\right|+\|\varphi\|_{\infty}\right)\right. \\
& \leq 2 \sqrt{N}\|\varphi\|_{\infty} \\
& \leq 2 C \sqrt{N}\|\varphi\|_{H^{s}} .
\end{aligned}
$$

This inequality combined with $\left\|Z_{t}^{N}\right\|_{H^{-s}}=\sup _{\varphi \neq 0, \varphi \in H^{s}} \frac{\left|\left(Z_{t}^{N}, \varphi\right)\right|}{\|\varphi\|_{H^{s}}}$, yields $\mathbb{E}\left(\sup _{t \geq 0}\left\|Z_{t}^{N}\right\|_{H^{-s}}^{2}\right) \leq 4 C N$.

The main result of this subsection is the next Theorem.

Theorem 6.6. The sequence $\left\{Z^{N}, N \geq 1\right\}$ converges in law in $C\left(\mathbb{R}_{+}, H^{-s}\right)$ towards $\left\{Z_{t}, t \geq 0\right\} \in C\left(\mathbb{R}_{+}, H^{-s}\right)$, where $\forall t \geq 0$, $Z_{t}=Z_{0}+\gamma \int_{0}^{t} \triangle Z_{r} d r+\widetilde{\mathcal{H}}_{t}$ and $\forall \varphi \in H^{s},(\widetilde{\mathcal{H}}, \varphi)$ is a centered Gaussian martingale whose predictable quadratic variation is given by $<(\widetilde{\mathcal{H}}, \varphi)>_{t}=2 \gamma \int_{0}^{t}\left(\mu_{r},(\nabla \varphi)^{2}\right) d r$. 
Before we prove this Theorem we first state a condition of Aldous-Rebolledo type for the tightness of a sequence of $\mathrm{H}$-valued càdlàg processes, where $\mathrm{H}$ is a Hilbert space (see definition 2.2.1, Corollary page 16, and the particular case 2.1.5 of [13]).

Proposition 6.7. Let $H$ be a separable Hilbert space, $\left(\vartheta^{n}\right)_{n}$ a sequence of $H$-valued càdlàg processes, their laws $\left(\widetilde{P}^{n}\right)$ form a tight sequence in $D\left(\mathbb{R}_{+}, H\right)$ if

$\left(T_{1}\right) \quad$ for each $t$ in a dense subset $\mathbb{\mathbb { N }}$ of $\mathbb{R}_{+}$, the sequence $\left(\vartheta_{t}^{n}\right)_{n}$ is tight in $H$

$\left(T_{2}\right) \quad \forall T>0, \forall \varepsilon_{1}, \varepsilon_{2}>0, \exists \delta>0, n_{0} \geq 1$ such that for any stopping times $\tau^{n} \leq T$

$$
\sup _{\substack{n \geq n_{0} \\ \theta \leq \delta}} \mathbb{P}\left(\left\|\vartheta_{\left(\tau^{n}+\theta\right)}^{n}-\vartheta_{\tau^{n}}^{n}\right\|_{H}>\varepsilon_{1}\right) \leq \varepsilon_{2}
$$

Note that if $\left(\vartheta^{n}\right)_{n}$ is a sequence of H-valued continuous processes, then a way to show that $\left(\vartheta^{n}\right)_{n}$ is tight is to prove that (T1) and (T2) are satisfied.

Let us now prove the following results which are useful for the proof of Theorem 6.6.

Proposition 6.8. The sequence $\widetilde{\mathcal{H}}^{N}$ converges in law in $C\left(\mathbb{R}_{+}, H^{-s}\right)$ towards $\widetilde{\mathcal{H}}$ where $\forall \varphi \in H^{s}$, $(\widetilde{\mathcal{H}}, \varphi)$ is a centered, continuous, Gaussian martingale having the same law as

$$
\begin{aligned}
\left(\widetilde{\mathcal{H}}_{t}, \varphi\right)= & \int_{0}^{t} \int_{\mathbb{T}^{2}} \frac{\partial \varphi}{\partial x_{1}}(x) \sqrt{2 \gamma f_{S}(r, x)} \mathcal{W}_{2}(d r, d x)+\int_{0}^{t} \int_{\mathbb{T}^{2}} \frac{\partial \varphi}{\partial x_{2}}(x) \sqrt{2 \gamma f_{S}(r, x)} \mathcal{W}_{3}(d r, d x) \\
& +\int_{0}^{t} \int_{\mathbb{T}^{2}} \frac{\partial \varphi}{\partial x_{1}}(x) \sqrt{2 \gamma f_{I}(r, x)} \mathcal{W}_{4}(d r, d x)+\int_{0}^{t} \int_{\mathbb{T}^{2}} \frac{\partial \varphi}{\partial x_{2}}(x) \sqrt{2 \gamma f_{I}(r, x)} \mathcal{W}_{5}(d r, d x) \\
& +\int_{0}^{t} \int_{\mathbb{T}^{2}} \frac{\partial \varphi}{\partial x_{1}}(x) \sqrt{2 \gamma\left(f(r, x)-f_{S}(r, x)-f_{I}(r, x)\right)} \mathcal{W}_{7}(d r, d x) \\
+ & \int_{0}^{t} \int_{\mathbb{T}_{2}} \frac{\partial \varphi}{\partial x_{2}}(x) \sqrt{2 \gamma\left(f(r, x)-f_{S}(r, x)-f_{I}(r, x)\right)} \mathcal{W}_{8}(d r, d x),
\end{aligned}
$$

where $\mathcal{W}_{1}, \mathcal{W}_{2}, \mathcal{W}_{3}, \mathcal{W}_{4}, \mathcal{W}_{5}, \mathcal{W}_{6}, \mathcal{W}_{7}, \mathcal{W}_{8}$ are independent spatio-temporal white noises.

Proof. We first establish the tightness of the sequence $\widetilde{\mathcal{H}}^{N}$, then show that all converging subsequences have the same limit which we shall identify. However, Theorem 2.15 of [19] restricted the tightness criterion of right-continuous martingales (Theorem 2.3.2 of [13]) to that of continuous martingales, thus to avoid repetition we obtain the tightness of $\widetilde{\mathcal{H}}^{N}$ by adapting the proof of Proposition 6.15 below. So by Prokhorov's theorem there exists a subsequence of $\widetilde{\mathcal{H}}^{N}$ still denoted $\widetilde{\mathcal{H}}^{N}$ which converge in law toward $\widetilde{\mathcal{H}}$. By adapting the proof of Lemma 6.17 below, we show that $\forall \varphi \in H^{s}$, the processes $(\widetilde{\mathcal{H}}, \varphi)$ is a centered, continuous martingale. On the other hand $\forall \varphi \in H^{s}$,

$$
<\widetilde{\mathcal{H}}^{N, \varphi}>_{t}=2 \gamma \int_{0}^{t}\left(\mu_{r}^{N},(\nabla \varphi)^{2}\right) d r \stackrel{P}{\rightarrow} 2 \gamma \int_{0}^{t}\left(\mu_{r},(\nabla \varphi)^{2}\right) d r=<(\widetilde{\mathcal{H}}, \varphi)>_{t}
$$

thus the quadractic variation $<(\widetilde{\mathcal{H}}, \varphi)>_{t}$ being deterministic, $(\widetilde{\mathcal{H}}, \varphi)$ is a centered, continuous, Gaussian martingale.

- Expression of $(\widetilde{\mathcal{H}}, \varphi)$ using the white noises.

We have $\widetilde{\mathcal{H}}_{t}^{N, \varphi}=\sqrt{\frac{2 \gamma}{N}} \sum_{i=1}^{N} \int_{0}^{t} \nabla \varphi\left(X_{r}^{i}\right) d X_{r}^{i}$

$$
\begin{aligned}
= & \sqrt{\frac{2 \gamma}{N}} \sum_{i=1}^{N} \int_{0}^{t} 1_{\left\{E_{r}^{i}=S\right\}} \nabla \varphi\left(X_{r}^{i}\right) d X_{r}^{i}+\sqrt{\frac{2 \gamma}{N}} \sum_{i=1}^{N} \int_{0}^{t} 1_{\left\{E_{r}^{i}=I\right\}} \nabla \varphi\left(X_{r}^{i}\right) d X_{r}^{i} \\
& +\sqrt{\frac{2 \gamma}{N}} \sum_{i=1}^{N} \int_{0}^{t} 1_{\left\{E_{r}^{i}=R\right\}} \nabla \varphi\left(X_{r}^{i}\right) d X_{r}^{i} .
\end{aligned}
$$

Furthemore

$$
<\widetilde{M}^{N, \phi}, \widetilde{\mathcal{H}}^{N, \varphi}>_{t}=2 \gamma \int_{0}^{t}\left(\mu_{r}^{S, N},(\nabla \varphi)(\nabla \phi)\right) d r \stackrel{P}{\rightarrow} 2 \gamma \int_{0}^{t}\left(\mu_{r}^{S},(\nabla \varphi)(\nabla \phi)\right) d r
$$




$$
\begin{aligned}
& <\widetilde{L}^{N, \psi}, \widetilde{\mathcal{H}}^{N, \varphi}>_{t}=2 \gamma \int_{0}^{t}\left(\mu_{r}^{I, N},(\nabla \varphi)(\nabla \phi)\right) d r \stackrel{P}{\rightarrow} 2 \gamma \int_{0}^{t}\left(\mu_{r}^{I},(\nabla \psi)(\nabla \phi)\right) d r, \\
& <\widetilde{\mathcal{H}}^{N, \varphi}>_{t}=2 \gamma \int_{0}^{t}\left(\mu_{r}^{N},(\nabla \phi)^{2}\right) d r \stackrel{P}{\rightarrow} 2 \gamma \int_{0}^{t}\left(\mu_{r},(\nabla \phi)^{2}\right) d r .
\end{aligned}
$$

Thus since $f(r,)-.f_{S}(r,)-.f_{I}(r,) \geq$.0 , for any $t \geq 0,\left(\widetilde{\mathcal{H}}_{t}, \varphi\right)$ satisfies $(6.4)$.

Proposition 6.9. There exists $C>0$, such that for any stopping times $\bar{\tau}<\infty$ a.s and $\theta>0$,

$$
\mathbb{E}\left(\left\|\int_{\bar{\tau}}^{\bar{\tau}+\theta} \Upsilon(\bar{\tau}+\theta-r) d \widetilde{\mathcal{H}}_{r}^{N}\right\|_{H^{-s}}^{2}\right) \leq C \theta .
$$

Proof. We first recall that $\left(f_{n_{1}, n_{2}}^{i}\right)_{i, n_{1}, n_{2}}$ (as defined is Proposition 2.2) is a family of eigenfonctions of the operator $\gamma \triangle$ associated to the family of eigenvalues $\left(-\lambda_{n_{1}, n_{2}}\right)_{n_{1}, n_{2}}$.

From (2.1) in Proposition 2.5 we see that $\nabla \Upsilon(t) f_{n_{1}, n_{2}}^{i}=e^{-t \lambda_{n_{1}, n_{2}}} \nabla f_{n_{1}, n_{2}}^{i}$, so by noticing that for any $\varphi \in H^{s+1} \subset C^{2}\left(\mathbb{T}^{2}\right)$,

one has

$$
\int_{\bar{\tau}}^{\bar{\tau}+\theta}\left(\Upsilon(\bar{\tau}+\theta-r) \varphi, d \widetilde{\mathcal{H}}_{r}^{N}\right)=\sqrt{\frac{2 \gamma}{N}} \sum_{i=1}^{N} \int_{\bar{\tau}}^{\bar{\tau}+\theta} \nabla \Upsilon(\bar{\tau}+\theta-r) \varphi\left(X_{r}^{i}\right) d B_{r}^{i},
$$

$$
\begin{aligned}
& \mathbb{E}\left(\left\|\int_{\bar{\tau}}^{\bar{\tau}+\theta} \Upsilon(\bar{\tau}+\theta-r) d \widetilde{\mathcal{H}}_{r}^{N}\right\|_{H^{-s}}^{2}\right)= \\
& =\sum_{i, n_{1}, n_{2}} \mathbb{E}\left(\left(\int_{\bar{\tau}}^{\bar{\tau}+\theta} \Upsilon(\bar{\tau}+\theta-r) \rho_{n_{1}, n_{2}}^{i, s}, d \widetilde{\mathcal{H}}_{r}^{N}\right)^{2}\right) \\
& =\sum_{i, n_{1}, n_{2}} \frac{2 \gamma}{N} \sum_{j=1}^{N} \mathbb{E}\left(\left(\int_{0}^{\theta} \nabla \Upsilon(\theta-r) \rho_{n_{1}, n_{2}}^{i, s}\left(X_{r+\tau}^{j}\right) d B_{r+\bar{\tau}}^{j}\right)^{2}\right) \\
& =2 \gamma \sum_{i, n_{1}, n_{2}} \mathbb{E}\left(\int_{0}^{\theta}\left(\mu_{r+\bar{\tau}}^{N},\left(1+\gamma \pi^{2}\left(n_{1}^{2}+n_{2}^{2}\right)\right)^{-s}\left(\nabla \Upsilon(\theta-r) f_{n_{1}, n_{2}}^{i}\right)^{2}\right) d r\right) \\
& =2 \gamma \sum_{i, n_{1}, n_{2}} \mathbb{E}\left(\int_{0}^{\theta}\left(\mu_{r+\bar{\tau}}^{N},\left(1+\gamma \pi^{2}\left(n_{1}^{2}+n_{2}^{2}\right)\right)^{-s} e^{-2(\theta-r) \lambda_{n_{1}, n_{2}}}\left(\nabla f_{n_{1}, n_{2}}^{i}\right)^{2}\right) d r\right) \\
& \leq 2 \gamma \mathbb{E}\left(\int_{0}^{\theta} \int_{\mathbb{T}^{2}} \sum_{i, n_{1}, n_{2}}\left(\nabla \rho_{n_{1}, n_{2}}^{i, s}(x)\right)^{2} \mu_{r+\bar{\tau}}^{N}(d x) d r\right) \\
& \leq 2 \gamma C \mathbb{E}\left(\int_{0}^{\theta} \int_{\mathbb{T}^{2}} \mu_{r+\bar{\tau}}^{N}(d x) d r\right) \\
& \leq 2 \gamma C \theta \text {, }
\end{aligned}
$$

where the second inequality follows from Lemma 8.1 below and the last one follows from the fact that $\int_{\mathbb{T}^{2}} \mu_{r+\bar{\tau}}^{N}(d x) \leq 1$.

Proposition 6.10. For all $T>0$,

$$
\sup _{N \geq 1} \sup _{0 \leq t \leq T} \mathbb{E}\left(\left\|Z_{t}^{N}\right\|_{H^{-s}}^{2}\right)<\infty .
$$

Proof. Recall that the semigroup $\Upsilon(t)$ generated by $\gamma \triangle$ satisfies $|\Upsilon(t)|_{\mathcal{L}\left(H^{-s}\right)} \leq 1$, where $\left.|\cdot|\right|_{\mathcal{L}\left(H^{-s}\right)}$ denotes the operator norm on $H^{-s}$.

From equation (6.1), we have $Z_{t}^{N}=\Upsilon(t) Z_{0}^{N}+\int_{0}^{t} \Upsilon(t-r) d \widetilde{\mathcal{H}}_{r}^{N}$.

Thus $\sup _{0 \leq t \leq T} \mathbb{E}\left(\left\|Z_{t}^{N}\right\|_{H^{-s}}^{2}\right) \leq 2 \mathbb{E}\left(\left\|Z_{0}^{N}\right\|_{H^{-s}}^{2}\right)+2 \sup _{0 \leq t \leq T} \mathbb{E}\left(\left\|\int_{0}^{t} \Upsilon(t-r) d \widetilde{\mathcal{H}}_{r}^{N}\right\|_{H^{-s}}^{2}\right)$.

Furthemore from Proposition 6.9 we deduce that $\sup _{0 \leq t \leq T} \mathbb{E}\left(\left\|\int_{0}^{t} \Upsilon(t-r) d \widetilde{\mathcal{H}}_{r}^{N}\right\|_{H^{-s}}^{2}\right) \leq C T$.

Combined with Proposition 4.1 in section 4, this show that $\sup \sup \mathbb{E}\left(\left\|Z_{t}^{N}\right\|_{H^{-s}}^{2}\right)<\infty$. 


\subsubsection{Proof of Theorem 6.6}

We first prove that $Z^{N}$ is tight in $C\left(\mathbb{R}_{+}, H^{-s}\right)$ then we show that all converging subsequences have the same limit.

Proposition 6.11. The sequence $Z^{N}$ is tight in $C\left(\mathbb{R}_{+}, H^{-s}\right)$.

Proof. We prove that $Z^{N}$ satisfies the conditions of Proposition 6.7 with $\mathrm{H}=H^{-s}$.

- Proof of (T1). It suffices to show that

$\forall t \geq 0, \forall \varepsilon>0$ there exists a compact subset $\mathcal{K}$ of $H^{-s}$ such that $\mathbb{P}\left(Z_{t}^{N} \notin \mathcal{K}\right)<\varepsilon$.

It follows from Proposition 6.10 that for each $2<\mathrm{s}^{\prime}<\mathrm{s}$, there exists $C$ such that,

$$
\sup _{N \geq 1} \sup _{0 \leq t<T} \mathbb{E}\left(\left\|Z_{t}^{N}\right\|_{H^{-s^{\prime}}}^{2}\right) \leq C \text {. }
$$

Thus since $\forall 2<s^{\prime}<s$ the embedding $H^{-s^{\prime}}\left(\mathbb{T}^{2}\right) \hookrightarrow H^{-s}\left(\mathbb{T}^{2}\right)$ is compact (see theorem 1.69 page 47 of [5]), $B_{H^{-s^{\prime}}}=\left\{\mu \in H^{-s^{\prime}} ;\|\mu\|_{H^{-s^{\prime}}} \leq R\right\}$ is a compact subset of $H^{-s}$. But

$$
\mathbb{P}\left(Z_{t}^{N} \notin B_{H^{-s^{\prime}}}\right)=\mathbb{P}\left(\left\|Z_{t}^{N}\right\|_{H^{-s^{\prime}}}>R\right) \leq \frac{1}{R^{2}} \mathbb{E}\left(\left\|Z_{t}^{N}\right\|_{H^{-s^{\prime}}}^{2}\right) \leq \frac{C}{R^{2}} .
$$

So by choosing $\mathrm{R}$ large enough we get the result.

- Proof of (T2). Let T>0, $\varepsilon_{1}, \varepsilon_{2}>0,\left(\tau^{N}\right)_{N}$ a family of stopping times such that $\tau^{N} \leq T$.

By noticing that $\forall 0 \leq u \leq t, Z_{t}^{N}=\Upsilon(t-u) Z_{u}^{N}+\int_{u}^{t} \Upsilon(t-r) d \widetilde{\mathcal{H}}_{r}^{N}$, we have

$Z_{\tau^{N}+\theta}^{N}-Z_{\tau^{N}}^{N}=\left(\Upsilon(\theta)-I_{d}\right) Z_{\tau^{N}}^{N}+\int_{\tau^{N}}^{\tau^{N}+\theta} \Upsilon\left(\tau^{N}+\theta-r\right) d \widetilde{\mathcal{H}}_{r}^{N}$.

We want to find $\delta>0$ and $N_{0} \geq 1$ such that

$$
\begin{aligned}
& \left.\sup _{N \geq N_{0} \delta \geq \theta} \sup _{\delta \geq \theta} \mathbb{P}\left(\Upsilon(\theta)-I_{d}\right) Z_{\tau^{N}}^{N} \|_{H^{-s}} \geq \varepsilon_{1}\right) \leq \varepsilon_{2}, \\
& \sup _{N \geq N_{0} \delta \geq \theta} \sup _{\delta \geq}\left(\left\|\int_{\tau^{N}}^{\tau^{N}+\theta} \Upsilon\left(\tau^{N}+\theta-r\right) d \widetilde{\mathcal{H}}_{r}^{N}\right\|_{H^{-s}} \geq \varepsilon_{1}\right) \leq \varepsilon_{2} .
\end{aligned}
$$

Proof of (6.5). Recall that $\left(\lambda_{n_{1}, n_{2}}\right)_{n_{1}, n_{2}}$ denotes the family of eigenvalues of the operator $-\gamma \triangle$. Let $m_{1}, m_{2} \in \mathbb{N}^{*}$, such that

$$
\left(\frac{32 \sup _{N \geq 1} \sup _{0 \leq t \leq T} \mathbb{E}\left(\left\|Z_{t}^{N}\right\|_{H^{-s+\sigma}}^{2}\right)}{\varepsilon_{1}^{2} \varepsilon_{2}}\right)^{\frac{1}{\sigma}}<\lambda_{m_{1}, m_{2}} \text {, for some } 0<\sigma<s-2 .
$$

Note that we can choose $m_{1}$ and $m_{2}$ such that (6.7) is satisfied since $\sup _{N \geq 1} \sup _{0 \leq t \leq T} \mathbb{E}\left(\left\|Z_{t}^{N}\right\|_{H^{-s+\sigma}}^{2}\right)<\infty$ for $0<\sigma<s-2$ (see Proposition 6.10) and $\left(\lambda_{m_{1}, m_{2}}\right)_{m_{1}, m_{2}}$ is a

non-decreasing sequence which converges to $+\infty$ as $m_{1} \longrightarrow \infty$ or $m_{2} \longrightarrow \infty$ (see Proposition $2.2)$.

Let $F_{m_{1}, m_{2}}$ denotes the sub-space of $H^{s}$ generated by $\left\{\rho^{0},\left(\rho_{\kappa_{1}, 0}^{i, s}, i \in\{5,6\}\right),\left(\rho_{0, \kappa_{2}}^{i, s}, i \in\{7,8\}\right),\left(\rho_{\kappa_{1}, \kappa_{2}}^{i, s}, i \in[|1,4|]\right), \kappa_{1}, \kappa_{2}\right.$ even and $\left.\kappa_{1} \leq m_{1}, \kappa_{2} \leq m_{2}\right\}$. Let $Z_{t \mid F_{m_{1}, m_{2}}}^{N}$ be the orthogonal projection of $Z_{t}^{N}$ on the dual space of $F_{m_{1}, m_{2}}$.

We have $\mathbb{P}\left(\left\|\left(\Upsilon(\theta)-I_{d}\right) Z_{\tau^{N}}^{N}\right\|_{H^{-s}} \geq \varepsilon_{1}\right) \leq \mathbb{P}\left(\left\|\left(\Upsilon(\theta)-I_{d}\right) Z_{\tau^{N} \mid F_{m_{1}, m_{2}}}^{N}\right\|_{H^{-s}} \geq \frac{\varepsilon_{1}}{2}\right)$

$$
+\mathbb{P}\left(\left\|\left(\Upsilon(\theta)-I_{d}\right)\left(Z_{\tau^{N}}^{N}-Z_{\tau^{N} \mid F_{m_{1}, m_{2}}}^{N}\right)\right\|_{H^{-s}} \geq \frac{\varepsilon_{1}}{2}\right) .
$$

Let us bound each of the two terms of the above right hand side.

$-\mathbb{P}\left(\left\|\left(\Upsilon(\theta)-I_{d}\right) Z_{\tau^{N} \mid F_{m_{1}, m_{2}}}^{N}\right\|_{H^{-s}} \geq \frac{\varepsilon_{1}}{2}\right) \leq \frac{4}{\varepsilon_{1}^{2}} \sup _{0 \leq t \leq T} \mathbb{E}\left(\left\|\left(\Upsilon(\theta)-I_{d}\right) Z_{t \mid F_{m_{1}, m_{2}}}^{N}\right\|_{H^{-s}}^{2}\right)$.

Furthemore from (2.1) in Proposition 2.5 $\Upsilon(t) f_{\kappa_{1}, \kappa_{2}}^{i}=e^{-\lambda_{\kappa_{1}, \kappa_{2}} t} f_{\kappa_{1}, \kappa_{2}}^{i}$, thus 


$$
\begin{aligned}
\left\|\left(\Upsilon(\theta)-I_{d}\right) Z_{t \mid F_{m_{1}, m_{2}}}^{N}\right\|_{H^{-s}}^{2} & =\sum_{i, \kappa 1, \kappa_{2}}^{m_{1}, m 2}\left(1+\gamma \pi^{2}\left(\kappa_{1}+\kappa_{2}\right)\right)^{-s}\left(\left(\Upsilon(\theta)-I_{d}\right) Z_{t}^{N}, f_{\kappa_{1}, \kappa_{2}}^{i}\right)^{2} \\
& =\sum_{i, \kappa 1, \kappa_{2}}^{m_{1}, m 2}\left(1+\gamma \pi^{2}\left(\kappa_{1}+\kappa_{2}\right)\right)^{-s}\left(Z_{t}^{N},\left(\Upsilon(\theta)-I_{d}\right) f_{\kappa_{1}, \kappa_{2}}^{i}\right)^{2} \\
& =\sum_{i, \kappa 1, \kappa_{2}}^{m_{1}, m 2}\left(e^{-\theta \lambda_{\kappa_{1}, \kappa_{2}}}-1\right)^{2}\left(1+\gamma \pi^{2}\left(\kappa_{1}+\kappa_{2}\right)\right)^{-s}\left(Z_{t}^{N}, f_{\kappa_{1}, \kappa_{2}}^{i}\right)^{2} \\
& \leq\left(e^{-\delta \lambda_{m_{1}, m_{2}}}-1\right)^{2}\left\|Z_{t}^{N}\right\|_{H^{-s}}^{2}
\end{aligned}
$$

hence $\mathbb{P}\left(\left\|\left(\Upsilon(\theta)-I_{d}\right) Z_{\tau^{N} \mid F_{m_{1}, m_{2}}}^{N}\right\|_{H^{-s}} \geq \frac{\varepsilon_{1}}{2}\right) \leq \frac{4\left(e^{-\delta \lambda_{m_{1}, m_{2}}}-1\right)^{2}}{\varepsilon_{1}^{2}} \sup _{N \geq 1} \sup _{0 \leq t \leq T} \mathbb{E}\left(\left\|Z_{t}^{N}\right\|_{H^{-s}}^{2}\right)$.

- Since $\left\|\Upsilon(t) Z_{t}^{N}\right\|_{H^{-s}} \leq\left\|Z_{t}^{N}\right\|_{H^{-s}}$

$$
\begin{aligned}
\mathbb{P}\left(\left\|\left(\Upsilon(\theta)-I_{d}\right)\left(Z_{\tau^{N}}^{N}-Z_{\tau^{N} \mid F_{m_{1}, m_{2}}}^{N}\right)\right\|_{H^{-s}} \geq \frac{\varepsilon_{1}}{2}\right) & \leq \mathbb{P}\left(\left\|\left(Z_{\tau^{N}}^{N}-Z_{\tau^{N} \mid F_{m_{1}, m_{2}}}^{N}\right)\right\|_{H^{-s}} \geq \frac{\varepsilon_{1}}{4}\right) \\
& \leq \frac{16}{\varepsilon_{1}^{2}} \sup _{0 \leq t \leq T} \mathbb{E}\left(\left\|\left(Z_{t}^{N}-Z_{t \mid F_{m_{1}, m_{2}}}^{N}\right)\right\|_{H^{-s}}^{2}\right) .
\end{aligned}
$$

On the other hand since $\left(\lambda_{\kappa_{1}, \kappa_{2}}\right)_{\kappa_{1}, \kappa_{2}}$ is a non-decreasing sequence, for any $0<\sigma<s-2$, one has

$$
\begin{aligned}
\left\|Z_{t}^{N}-Z_{t \mid F_{m_{1}, m_{2}}}^{N}\right\|_{H^{-s}}^{2} & =\left\|\left(I_{d}-\gamma \triangle\right)^{\frac{-\sigma}{2}}\left(I_{d}-\gamma \triangle\right)^{\frac{\sigma}{2}}\left(Z_{t}^{N}-Z_{t \mid F_{m_{1}, m_{2}}}^{N}\right)\right\|_{H^{-s}}^{2} \\
& =\sum_{i, \kappa_{1}, \kappa_{2}} \lambda_{\kappa_{1}, \kappa_{2}}^{-s}\left(\left(I_{d}-\gamma \triangle\right)^{\frac{-\sigma}{2}}\left(I_{d}-\gamma \triangle\right)^{\frac{\sigma}{2}}\left(Z_{t}^{N}-Z_{t \mid F_{m_{1}, m_{2}}}^{N}\right), f_{\kappa_{1}, \kappa_{2}}^{i}\right)^{2} \\
& =\sum_{i, \kappa_{1}, \kappa_{2}} \lambda_{\kappa_{1}, \kappa_{2}}^{-s}\left(Z_{t}^{N}-Z_{t \mid F_{m_{1}, m_{2}}}^{N},\left(I_{d}-\gamma \triangle\right)^{\frac{\sigma}{2}}\left(I_{d}-\gamma \triangle\right)^{\frac{-\sigma}{2}} f_{\kappa_{1}, \kappa_{2}}^{i}\right)^{2} \\
& =\sum_{i, \kappa_{1}, \kappa_{2}} \lambda_{\kappa_{1}, \kappa_{2}}^{-s+\sigma} \lambda_{\kappa_{1}, \kappa_{2}}^{-\sigma}\left(Z_{t}^{N}-Z_{t \mid F_{m_{1}, m_{2}}}^{N}, f_{\kappa_{1}, \kappa_{2}}^{i}\right)^{2} \\
& \leq \lambda_{m_{1}, m_{2}}^{-\sigma} \sum_{i, \kappa_{1}=m_{1}+1, \kappa_{2}=m_{2}+1}^{\infty} \lambda_{\kappa_{1}, \kappa_{2}}^{-s+\sigma}\left(Z_{t}^{N}, f_{\kappa_{1}, \kappa_{2}}^{i}\right)^{2} \\
& \leq \lambda_{m_{1}, m_{2}}^{-\sigma}\left\|Z_{t}^{N}\right\|_{H^{-s+\sigma}}^{2} .
\end{aligned}
$$

Thus

$$
\mathbb{P}\left(\left\|\left(\Upsilon(\theta)-I_{d}\right)\left(Z_{\tau^{N}}^{N}-Z_{\tau^{N} \mid F_{m_{1}, m_{2}}}^{N}\right)\right\|_{H^{-s}} \geq \frac{\varepsilon_{1}}{2}\right) \leq \frac{16 \lambda_{m_{1}, m_{2}}^{-\sigma}}{\varepsilon_{1}^{2}} \sup _{N \geq 10 \leq t \leq T} \sup \mathbb{E}\left(\left\|Z_{t}^{N}\right\|_{H^{-s+\sigma}}^{2}\right) .
$$

So from (6.7), (6.8) and (6.9) we deduce (6.5).

Proof of (6.6). From Proposition 6.9, we have

$$
\begin{aligned}
& \mathbb{P}\left(\left\|\int_{\tau^{N}}^{\tau^{N}+\theta} \Upsilon\left(\tau^{N}+\theta-r\right) d \widetilde{\mathcal{H}}_{r}^{N}\right\|_{H^{-s}} \geq \varepsilon_{1}\right) \leq \frac{1}{\varepsilon_{1}^{2}} \mathbb{E}\left(\left\|\int_{\tau^{N}}^{\tau^{N}+\theta} \Upsilon\left(\tau^{N}+\theta-r\right) d \widetilde{\mathcal{H}}_{r}^{N}\right\|_{H^{-s}}^{2}\right) \\
& \leq \frac{C}{\varepsilon_{1}^{2}} \delta .
\end{aligned}
$$

(6.6) follows. (T1), (T2) are proved, hence $\left(Z^{N}\right)_{N}$ is tight in $C\left(\mathbb{R}_{+}, H^{-s}\right)$.

We end the proof of Theorem 6.6 by showing the next Proposition.

Proposition 6.12. Every limit point $Z$ of $Z^{N}$ is solution of

$$
Z_{t}=\Upsilon(t) Z_{0}+\int_{0}^{t} \Upsilon(t-r) d \widetilde{\mathcal{H}}_{r}
$$

Proof. We have $Z_{t}^{N}=\Upsilon(t) Z_{0}^{N}+\int_{0}^{t} \Upsilon(t-r) d \widetilde{\mathcal{H}}_{r}^{N}$. Furthemore

- according to Proposition $6.8 \int_{0}^{t} \Upsilon(t-r) d \widetilde{\mathcal{H}}_{r}^{N} \stackrel{L}{\rightarrow} \int_{0}^{t} \Upsilon(t-r) d \widetilde{\mathcal{H}}_{r}$;

- according to Theorem $4.2 \Upsilon(t) Z_{0}^{N} \stackrel{L}{\rightarrow} \Upsilon(t) Z_{0}$.

Hence the result. 


\subsection{Convergence of $\left(U^{N}, V^{N}\right)_{N \geq 1}$}

We recall that $\left(U^{N}, V^{N}\right)$ satisfies the equations (6.2) and (6.3).

The following Lemma is proved by the same argument as used in the proof of Lemma 6.5.

Lemma 6.13. For all $N \geq 1$, the processes $U^{N}$ and $V^{N}$ belong to $D\left(\mathbb{R}_{+}, H^{-s}\right)$.

We now state the main result of this section.

Theorem 6.14. Under (H2), the sequence of processes $\left(U^{N}, V^{N}\right)_{N \geq 1}$ converges in law in $\left(D\left(\mathbb{R}_{+}, H^{-s}\right)\right)^{2}$ to the pair of processes $(U, V)$ which belongs to $\left(C\left(\mathbb{R}_{+}, H^{-s}\right)\right)^{2}$ and satisfies

$$
\begin{gathered}
U_{t}=U_{0}+\gamma \int_{0}^{t} \triangle U_{r} d r+\beta \int_{0}^{t}\left(G_{r}^{S, I}\right)^{*} Z_{r} d r-\beta \int_{0}^{t}\left(G_{r}^{I}\right)^{*} U_{r} d r-\beta \int_{0}^{t}\left(G_{r}^{S}\right)^{*} V_{r} d r+W_{t}^{1} \\
V_{t}=V_{0}+\gamma \int_{0}^{t} \triangle V_{r} d r-\beta \int_{0}^{t}\left(G_{r}^{S, I}\right)^{*} Z_{r} d r+\beta \int_{0}^{t}\left(G_{r}^{I}\right)^{*} U_{r} d r+\int_{0}^{t}\left(\beta\left(G_{r}^{S}\right)^{*}-\alpha I_{d}\right) V_{r} d r+W_{t}^{2},
\end{gathered}
$$

where $\forall \varphi, \psi \in H^{s},\left(\left(W^{1}, \varphi\right),\left(W^{2}, \psi\right)\right)$ is a centered continuous Gaussian martingale verifying

$$
\begin{aligned}
& <\left(W^{1}, \varphi\right)>_{t}=\beta \int_{0}^{t}\left(\mu_{r}^{S}, \varphi^{2}\left(\mu_{r}^{I}, \frac{K}{\left(\mu_{r}, K\right)}\right)\right) d r+2 \gamma \int_{0}^{t}\left(\mu_{r}^{S},(\nabla \varphi)^{2}\right) d r \\
& <\left(W^{2}, \psi\right)>_{t}=\beta \int_{0}^{t}\left(\mu_{r}^{S}, \psi^{2}\left(\mu_{r}^{I}, \frac{K}{\left(\mu_{r}, K\right)}\right)\right) d r+2 \gamma \int_{0}^{t}\left(\mu_{r}^{I},(\nabla \psi)^{2}\right) d r+\alpha \int_{0}^{t}\left(\mu_{r}^{I}, \psi^{2}\right) d r, \\
& <\left(W^{1}, \varphi\right),\left(W^{2}, \psi\right)>_{t}=-\beta \int_{0}^{t}\left(\mu_{r}^{S}, \varphi \psi\left(\mu_{r}^{I}, \frac{K}{\left(\mu_{r}, K\right)}\right)\right) d r .
\end{aligned}
$$

The proof of this Theorem is the aim of this subsection, however let us first prove the following preliminary results.

Proposition 6.15. Both sequences $\left(\widetilde{M}^{N}\right)_{N \geq 1}$ and $\left(\widetilde{L}^{N}\right)_{N \geq 1}$ are tight in $D\left(\mathbb{R}_{+}, H^{-s}\right)$.

Proof. - Tightness of $\left(\widetilde{M}^{N}\right)_{N \geq 1}$.

We prove that $\left(\widetilde{M}^{N}\right)_{N \geq 1}$ satisfies the conditions (T1) and (T2) of the Proposition 6.7.

- Based on Corollary 6.4, we deduce (T1) by the same argument as used in the proof of (T1) in Theorem 6.6.

- Proof of (T2). First note that $<\widetilde{M}^{N, \varphi}>_{t}=\int_{0}^{t} A_{r}^{N}(\varphi) d r$, where

$$
A_{r}^{N}(\varphi)=\beta\left(\mu_{r}^{S, N}, \varphi^{2}\left(\mu_{r}^{I, N}, \frac{K}{\left(\mu_{r}^{N}, K\right)}\right)\right)+2 \gamma\left(\mu_{r}^{S, N},(\nabla \varphi)^{2}\right) .
$$

According to Theorem 2.3.2 in [13] it is enough to prove that $\forall T>0 \quad \forall \varepsilon_{1}, \varepsilon_{2}>0 \quad \exists \delta>0, N_{0} \geq 1$ such that for any stopping times $\tau^{N} \leq T$,

$$
\sup _{N \geq N_{0}} \sup _{\theta \leq \delta} \mathbb{P}\left(\left|<\widetilde{M}^{N}>_{\left(\tau^{N}+\theta\right)}-<\widetilde{M}^{N}>_{\tau^{N}}\right|>\varepsilon_{1}\right)<\varepsilon_{2}
$$

where $<\widetilde{M}>$ is the increasing, continuous process such that, $\left\|\widetilde{M}_{t}\right\|_{H^{-s}}^{2}-<\widetilde{M}>_{t}$ is a martingale. It is the trace of $\ll \widetilde{M} \gg$ which is the continuous increasing operator valued process such that $\left\{\widetilde{M}_{t} \otimes \widetilde{M}_{t}-\ll \widetilde{M} \gg_{t}, t \geq 0\right\}$ is a martingale.

Let $T>0, \varepsilon_{1}, \varepsilon_{2}>0$, from Lemma 8.1 below, we have

$$
\begin{aligned}
& \left|<\widetilde{M}^{N}>_{\left(\tau^{N}+\theta\right)}-<\widetilde{M}^{N}>_{\tau^{N}}\right|=\left|\sum_{i, n_{1}, n_{2}}\left\{<\widetilde{M}^{N, \rho_{n_{1}, n_{2}}^{i, s}}>_{\left(\tau^{N}+\theta\right)}-<\widetilde{M}^{N, \rho_{n_{1}, n_{2}}^{i, s}}>_{\tau^{N}}\right\}\right| \\
& =\left|\sum_{i, n_{1}, n_{2}} \int_{\tau^{N}}^{\left(\tau^{N}+\theta\right)} A_{r}^{N}\left(\rho_{n_{1}, n_{2}}^{i, s}\right) d r\right|
\end{aligned}
$$




$$
\begin{aligned}
& =\left|\sum_{i, n_{1}, n_{2}} \int_{0}^{\theta} A_{\left(\tau^{N}+r\right)}^{N}\left(\rho_{n_{1}, n_{2}}^{i, s}\right) d r\right| \\
& \leq C \int_{0}^{\theta}\left\{\int_{\mathbb{T}^{2}} \frac{\int_{\mathbb{T}^{2}} K(x, y) \mu_{\tau^{N}+r}^{S, N}(d x)}{\int_{\mathbb{T}^{2}} K\left(x^{\prime}, y\right) \mu_{\tau^{N}+r}^{N}\left(d x^{\prime}\right)} \mu_{\tau^{N}+r}^{I, N}(d y)+\int_{\mathbb{T}^{2}} \mu_{\tau^{N}+r}^{S, N}(d x)\right\} d r \\
& \leq \delta C .
\end{aligned}
$$

Hence it follows from the Markov inequality that

$$
\begin{aligned}
\mathbb{P}\left(\left|<\widetilde{M}^{N}>_{\left(\tau^{N}+\theta\right)}-<\widetilde{M}^{N}>_{\tau^{N}}\right|>\varepsilon_{1}\right) & \leq \frac{\mathbb{E}\left(\left|<\widetilde{M}^{N}>_{\left(\tau^{N}+\theta\right)}-<\widetilde{M}^{N}>_{\tau^{N}}\right|\right)}{\varepsilon_{1}} \\
& \leq \frac{C \delta}{\varepsilon_{1}} .
\end{aligned}
$$

(T1) and (T2) proved, we conclude that $\left(\widetilde{M}^{N}\right)_{N \geq 1}$ is tight in $D\left(\mathbb{R}_{+}, H^{-s}\right)$. The same argument yields the tightness of $\left(\widetilde{L}^{N}\right)_{N \geq 1}$ in $D\left(\mathbb{R}_{+}, H^{-s}\right)$.

Proposition 6.16. Every limit point $\left(W^{1}, W^{2}\right)$ of the sequence $\left(\widetilde{M}^{N}, \widetilde{L}^{N}\right)_{N \geq 1}$ is an element of $C\left(\mathbb{R}_{+}, H^{-s}\right) \times C\left(\mathbb{R}_{+}, H^{-s}\right)$.

Proof. We prove that the processes $W^{1}$ and $W^{2}$ are continuous.

Since Proposition 3.26 page 315 in [12] concerning the continuity of the limit in law of $\mathbb{R}^{n}$-valued càdlàg processes can be adapded to càdlàg processes that take values in an arbitrary Hilbert space, to prove that $W^{1}$ is continuous, it is enough to show that

$$
\forall T>0 \quad \forall \varepsilon>0 \quad \lim _{N \rightarrow \infty} \mathbb{P}\left(\sup _{0 \leq t \leq T}\left\|\widetilde{M}_{t}^{N}-\widetilde{M}_{t^{-}}^{N}\right\|_{H^{-s}}>\varepsilon\right)=0 .
$$

Let $T>0, \varphi \in H^{s+1}$, since the jump of $M^{N, \varphi}$ and $\left(\mu^{S, N}, \varphi\right)$ happen at the same time, we have

$$
\left|\widetilde{M}_{t}^{N, \varphi}-\widetilde{M}_{t^{-}}^{N, \varphi}\right| \leq \frac{C\|\varphi\|_{H^{s}}}{\sqrt{N}} .
$$

Thus $\mathbb{P}\left(\sup _{0 \leq t \leq T}\left\|\widetilde{M}_{t}^{N}-\widetilde{M}_{t^{-}}^{N}\right\|_{H^{-s}}>\varepsilon\right) \leq \frac{1}{\varepsilon} \mathbb{E}\left(\sup _{0 \leq t \leq T}\left\|\widetilde{M}_{t}^{N}-\widetilde{M}_{t^{-}}^{N}\right\|_{H^{-s}}\right) \leq \frac{C}{\varepsilon \sqrt{N}}$,

$$
\text { so } \lim _{N \longrightarrow \infty} \mathbb{P}\left(\sup _{0 \leq t \leq T}\left\|\widetilde{M}_{t}^{N}-\widetilde{M}_{t^{-}}^{N}\right\|_{H^{-s}}>\varepsilon\right)=0 \text {. }
$$

The same argument yields the fact that $W^{2}$ is continuous.

Lemma 6.17. Every limit point $\left(W^{1}, W^{2}\right)$ of the sequence $\left(\widetilde{M}^{N}, \widetilde{L}^{N}\right)_{N \geq 1}$ is such that for any $\varphi, \psi \in H^{s+1},\left(\left(W^{1}, \varphi\right),\left(W^{2}, \psi\right)\right)$ is a martingale.

Proof. - Martingale property of $\left(W^{1}, \varphi\right)$

A sufficient condition for $\left(W^{1}, \varphi\right)$ to be a martingale, is that, for all $k \in \mathbb{N}^{*}, \Phi_{k} \in C_{b}\left(\mathbb{R}^{k}\right)$, $\varphi_{1}, \varphi_{2}, \ldots \varphi_{k} \in H^{s}$, and $0 \leq s_{0}<s_{1}<s_{2}<s_{3}<\ldots .<s_{k} \leq s<t$

$$
\mathbb{E}\left(\phi\left(\left(W^{1}, \varphi\right)\right)\right)=0,
$$

where $\phi\left(\left(W^{1}, \varphi\right)\right)=\Phi_{k}\left(\left(W_{s_{1}}^{1}, \varphi_{1}\right),\left(W_{s_{2}}^{1}, \varphi_{2}\right), \ldots \ldots \ldots \ldots,\left(W_{s_{k}}^{1}, \varphi_{k}\right)\right)\left(\left(W_{t}^{1}, \varphi\right)-\left(W_{s}^{1}, \varphi\right)\right)$.

However given that $\widetilde{M}^{N, \varphi}$ is a martingale, $\mathbb{E}\left(\phi\left(\widetilde{M}^{N, \varphi}\right)\right)=0$, moreover $\phi$ is continuous thus,

$$
\phi\left(\widetilde{M}^{N, \varphi}\right) \stackrel{L}{\rightarrow} \phi\left(\left(W^{1}, \varphi\right)\right), \text { as } N \rightarrow \infty .
$$

On the other hand $\phi\left(\widetilde{M}^{N, \varphi}\right)$ is uniformly integrable since $\mathbb{E}\left[\left(\phi\left(\widetilde{M}^{N, \varphi}\right)^{2}\right] \leq C\right.$, hence $\mathbb{E}\left(\phi\left(\left(W^{1}, \varphi\right)\right)\right)=\lim _{N \rightarrow \infty} \mathbb{E}\left[\phi\left(\widetilde{M}^{N, \varphi}\right)\right]=0$.

So we conclude that $\left(W^{1}, \varphi\right)$ is a martingale. A similar argument shows that $\left(W^{2}, \psi\right)$ is a martingale. 
Proposition 6.18. The sequence $\left(\widetilde{M}^{N}, \widetilde{L}^{N}\right)_{N \geq 1}$ converges in law in $\left(D\left(\mathbb{R}_{+}, H^{-s}\right)\right)^{2}$ towards the processes $\left(W^{1}, W^{2}\right) \in C\left(\mathbb{R}_{+}, H^{-s}\right) \times C\left(\mathbb{R}_{+}, H^{-s}\right)$ where $\forall \varphi, \psi \in H^{s},\left(\left(W^{1}, \varphi\right),\left(W^{2}, \psi\right)\right)$ is a centered Gaussian martingale having the same law as:

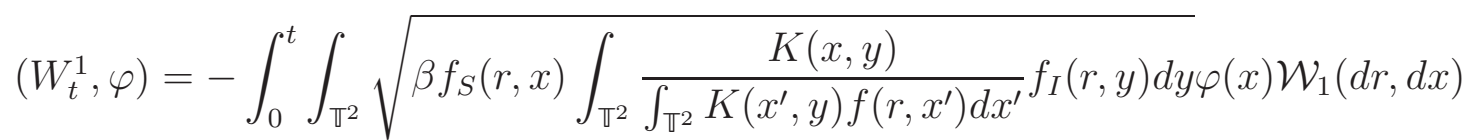

$$
\begin{aligned}
& +\int_{0}^{t} \int_{\mathbb{T}^{2}} \frac{\partial \varphi}{\partial x_{1}}(x) \sqrt{2 \gamma f_{S}(r, x)} \mathcal{W}_{2}(d r, d x)+\int_{0}^{t} \int_{\mathbb{T}^{2}} \frac{\partial \varphi}{\partial x_{2}}(x) \sqrt{2 \gamma f_{S}(r, x)} \mathcal{W}_{3}(d r, d x), \\
& \left(W_{t}^{2}, \psi\right)=\int_{0}^{t} \int_{\mathbb{T}^{2}} \sqrt{\beta f_{S}(r, x) \int_{\mathbb{T}^{2}} \frac{K(x, y)}{\int_{\mathbb{T}^{2}} K\left(x^{\prime}, y\right) f\left(r, x^{\prime}\right) d x^{\prime}} f_{I}(r, y) d y \psi}(x) \mathcal{W}_{1}(d r, d x) \\
& +\int_{0}^{t} \int_{\mathbb{T}^{2}} \frac{\partial \psi}{\partial x_{1}}(x) \sqrt{2 \gamma f_{I}(r, x)} \mathcal{W}_{4}(d r, d x)+\int_{0}^{t} \int_{\mathbb{T}^{2}} \frac{\partial \psi}{\partial x_{2}}(x) \sqrt{2 \gamma f_{I}(r, x)} \mathcal{W}_{5}(d r, d x) \\
& +\int_{0}^{t} \int_{\mathbb{T}^{2}} \psi(x) \sqrt{\alpha f_{I}(r, x)} \mathcal{W}_{6}(d r, d x)
\end{aligned}
$$

where $\mathcal{W}_{1}, \mathcal{W}_{2}, \mathcal{W}_{3}, \mathcal{W}_{4}, \mathcal{W}_{5}, \mathcal{W}_{6}$ are independent spatio-temporal white noises defined in Proposition 6.8 .

Proof. From Proposition $6.15\left(\widetilde{M}^{N}, \widetilde{L}^{N}\right)_{N \geq 1}$ is tight in $D\left(\mathbb{R}_{+}, H^{-s}\right) \times D\left(\mathbb{R}_{+}, H^{-s}\right)$,

hence according to Prokhorov's theorem there exists a subsequence still denoted $\left(\widetilde{M}^{N}, \widetilde{L}^{N}\right)_{N \geq 1}$ which converges in law in $\left(D\left(\mathbb{R}_{+}, H^{-s}\right)\right)^{2}$ towards $\left(W^{1}, W^{2}\right)$. By Proposition 6.16 and Lemma 6.17, $\forall \varphi, \psi \in H^{s},\left(\left(W^{1}, \varphi\right),\left(W^{2}, \psi\right)\right)$ is a continuous martingale, thus we end the proof of proposition 6.18 by showing that the centered, continuous martingale $\left(\left(W^{1}, \varphi\right),\left(W^{2}, \psi\right)\right)$ is Gaussian and satisfies (6.12) and (6.13).

We have

$$
\begin{aligned}
& \widetilde{M}_{t}^{N, \varphi}=-\frac{1}{\sqrt{N}} \sum_{i=1}^{N} \int_{0}^{t} \int_{0}^{\infty} 1_{\left\{E_{r^{-}}^{i}=S\right\}} \varphi\left(X_{r}^{i}\right) 1_{\left\{u \leq \frac{\beta}{N} \sum_{j=1}^{N} \frac{K\left(X_{r}^{i}, X_{r}^{j}\right)}{\mu_{r}^{N}\left(K\left(, X_{r}^{j}\right)\right)} 1_{\left\{E_{r}^{j}=I\right\}}\right\}} \bar{M}^{i}(d r, d u) \\
& +\sqrt{\frac{2 \gamma}{N}} \sum_{i=1}^{N} \int_{0}^{t} 1_{\left\{E_{r}^{i}=S\right\}} \nabla \varphi\left(X_{r}^{i}\right) d B_{r}^{i} \\
& =-M_{t}^{1, N, \varphi}+M_{t}^{2, N, \varphi}, \\
& \left.\widetilde{L}_{t}^{N, \varphi}=\frac{1}{\sqrt{N}} \sum_{i=1}^{N} \int_{0}^{t} \int_{0}^{\infty} 1_{\left\{E_{r^{-}}^{i}=S\right\}} \varphi\left(X_{r}^{i}\right) 1_{\left\{u \leq \frac{\beta}{N} \sum_{j=1}^{N} \frac{K\left(X_{r}^{i}, X_{r}^{j}\right)}{\mu_{r}^{N}\left(K\left(, X_{r}^{j}\right)\right)} 1_{\left\{E_{r}^{j}=I\right\}}\right\}} \bar{M}^{i}(d r, d u)\right)+ \\
& +\sqrt{\frac{2 \gamma}{N}} \sum_{i=1}^{N} \int_{0}^{t} 1_{\left\{E_{r}^{i}=I\right\}} \nabla \varphi\left(X_{r}^{i}\right) d B_{r}^{i}-\frac{1}{\sqrt{N}} \sum_{i=1}^{N} \int_{0}^{t} \int_{0}^{\alpha} 1_{\left\{E_{r^{-}}^{i}=I\right\}} \varphi\left(X_{r}^{i}\right) \bar{Q}^{i}(d r, d u) \\
& =M_{t}^{1, N, \varphi}+M_{t}^{3, N, \varphi}+M_{t}^{4, N, \varphi} \text {. }
\end{aligned}
$$

Consider for $\varphi, \psi \in H^{s+1}\left(\mathbb{T}^{2}\right)$ the following sequence of martingales

$$
\widetilde{M}_{t}^{N, \varphi}+\widetilde{L}_{t}^{N, \psi}=-M_{t}^{1, N, \varphi}+M_{t}^{2, N, \varphi}+M_{t}^{1, N, \psi}+M_{t}^{3, N, \psi}+M_{t}^{4, N, \psi} .
$$

The martingales $M_{t}^{1, N, \varphi}, M_{t}^{2, N, \varphi}, M_{t}^{3, N, \psi}, M_{t}^{4, N, \psi}$ being two by two orthogonal,

$$
\begin{aligned}
<\widetilde{M}^{N, \varphi}+\widetilde{L}^{N, \psi}>_{t}= & <M^{1, N, \varphi}>_{t}+<M^{2, N, \varphi}>_{t}+<M^{1, N, \psi}>_{t}+<M^{3, N, \psi}>_{t}+<M^{4, N, \psi}>_{t} \\
& -2<M^{1, N, \varphi}, M^{1, N, \psi}>_{t} .
\end{aligned}
$$

In addition we have the following convergences in probability

$$
\begin{aligned}
& <M^{1, N, \varphi}>_{t} \stackrel{P}{\rightarrow} \beta \int_{0}^{t}\left(\mu_{r}^{S}, \varphi^{2}\left(\mu_{r}^{I}, \frac{K}{\left(\mu_{r}, K\right)}\right)\right) d r,<M^{2, N, \varphi}>_{t} \stackrel{P}{\rightarrow} 2 \gamma \int_{0}^{t}\left(\mu_{r}^{S},(\nabla \varphi)^{2}\right) d r \\
& <M^{3, N, \psi}>_{t} \stackrel{P}{\rightarrow} 2 \gamma \int_{0}^{t}\left(\mu_{r}^{I},(\nabla \psi)^{2}\right) d r,<M^{4, N, \psi}>_{t} \stackrel{P}{\rightarrow} \alpha \int_{0}^{t}\left(\mu_{r}^{I}, \psi^{2}\right) d r .
\end{aligned}
$$


On the other hand:

- $\widetilde{M}^{N, \varphi}+\widetilde{L}^{N, \psi} \stackrel{L}{\rightarrow}\left(W^{1}, \varphi\right)+\left(W^{2}, \psi\right)$ along a subsequence since

$\left(\widetilde{M}^{N, \varphi}, \widetilde{L}^{N, \psi}\right) \stackrel{L}{\rightarrow}\left(\left(W^{1}, \varphi\right),\left(W^{2}, \psi\right)\right)$.

- $\left(W^{1}, \varphi\right)+\left(W^{2}, \psi\right)$ is a continuous martingale since $\left(W^{1}, \varphi\right)$ and $\left(W^{2}, \psi\right)$ have this property.

Thus $\left(W^{1}, \varphi\right)+\left(W^{2}, \psi\right)$ is a time changed Brownian motion.

The quadratic variation

$$
\begin{aligned}
<\left(W^{1}, \varphi\right)+\left(W^{2}, \psi\right)>_{t} \\
=\int_{0}^{t} \beta\left[\left(\mu_{r}^{S}, \varphi^{2}\left(\mu_{r}^{I}, \frac{K}{\left(\mu_{r}, K\right)}\right)\right)+\left(\mu_{r}^{S}, \psi^{2}\left(\mu_{r}^{I}, \frac{K}{\left(\mu_{r}, K\right)}\right)\right)-2\left(\mu_{r}^{S}, \varphi \psi\left(\mu_{r}^{I}, \frac{K}{\left(\mu_{r}, K\right)}\right)\right)\right] d r \\
\quad+\int_{0}^{t}\left\{2 \gamma\left(\mu_{r}^{S},(\nabla \varphi)^{2}\right)+2 \gamma\left(\mu_{r}^{I},(\nabla \psi)^{2}\right)+\alpha\left(\mu_{r}^{I}, \psi^{2}\right)\right\} d r
\end{aligned}
$$

of $\left(W^{1}, \varphi\right)+\left(W^{2}, \psi\right)$ being deterministic then we conclude that $\left(W^{1}, \varphi\right)+\left(W^{2}, \psi\right)$ is a Gaussian martingale having the same law as

$$
\begin{aligned}
& \left(W_{t}^{1}, \varphi\right)+\left(W_{t}^{2}, \psi\right)= \\
& -\int_{0}^{t} \int_{\mathbb{T}^{2}} \sqrt{\beta f_{S}(r, x) \int_{\mathbb{T}^{2}} \frac{K(x, y)}{\int_{\mathbb{T}^{2}} K\left(x^{\prime}, y\right) f\left(r, x^{\prime}\right) d x^{\prime}} f_{I}(r, y) d y}(\varphi(x)-\psi(x)) \mathcal{W}_{1}(d r, d x) \\
& +\int_{0}^{t} \int_{\mathbb{T}^{2}} \frac{\partial \varphi}{\partial x_{1}}(x) \sqrt{2 \gamma f_{S}(r, x)} \mathcal{W}_{2}(d r, d x)+\int_{0}^{t} \int_{\mathbb{T}^{2}} \frac{\partial \varphi}{\partial x_{2}}(x) \sqrt{2 \gamma f_{S}(r, x)} \mathcal{W}_{3}(d r, d x) \\
& +\int_{0}^{t} \int_{\mathbb{T}^{2}} \frac{\partial \psi}{\partial x_{1}}(x) \sqrt{2 \gamma f_{I}(r, x)} \mathcal{W}_{4}(d r, d x)+\int_{0}^{t} \int_{\mathbb{T}^{2}} \frac{\partial \psi}{\partial x_{2}}(x) \sqrt{2 \gamma f_{I}(r, x)} \mathcal{W}_{5}(d r, d x) \\
& +\int_{0}^{t} \int_{\mathbb{T}^{2}} \psi(x) \sqrt{\alpha f_{I}(r, x)} \mathcal{W}_{6}(d r, d x)
\end{aligned}
$$

where $\mathcal{W}_{1}, \mathcal{W}_{2}, \mathcal{W}_{3}, \mathcal{W}_{4}, \mathcal{W}_{5}, \mathcal{W}_{6}$ are independent spatio-temporal white noises defined in Proposition 6.8 .

So taking $\psi \equiv 0, \varphi \equiv 0$ respectively in the above equation we see that $\left(W^{1}, \varphi\right)$ and $\left(W^{2}, \psi\right)$ satisfy (6.12) and (6.13).

\subsubsection{Proof of Theorem 6.14}

We first prove that $U^{N}$ and $V^{N}$ are tight in $D\left(\mathbb{R}_{+}, H^{-s}\right)$ then we show that all converging subsequences of $\left(U^{N}, V^{N}\right)_{N \geq 1}$ have the same limit which we shall identify. Let us recall the following embeddings which follow from Proposition 2.4, Lemma 2.6 above, and from Theorem 1.69 page 47 of [5].

- If $s^{\prime}>1$ then $H^{s^{\prime}}\left(\mathbb{T}^{2}\right) \subset C\left(\mathbb{T}^{2}\right)$ and for all $\varphi \in H^{s^{\prime}}, \Upsilon(t) \varphi \in H^{s^{\prime}}$ and $\|\Upsilon(t) \varphi\|_{H^{s^{\prime}}} \leq\|\varphi\|_{H^{s^{\prime}}}$ furthemore if $\phi, \psi \in H^{s^{\prime}}$ there exists $C>0$ such that $\|\phi \psi\|_{H^{s^{\prime}}} \leq C\|\phi\|_{H^{s^{\prime}}}\|\psi\|_{H^{s^{\prime}}}$.

- If $s^{\prime}>2$ then, $H^{s^{\prime}+1}\left(\mathbb{T}^{2}\right) \subset C^{2}\left(\mathbb{T}^{2}\right)$.

$-\forall s_{1}, s_{2} \in \mathbb{R}$ such that $s_{1}>s_{2}$ the embedding $H^{s_{1}}\left(\mathbb{T}^{2}\right) \hookrightarrow H^{s_{2}}\left(\mathbb{T}^{2}\right)$ is compact.

Proposition 6.19. There exists a constant $C>0$ such that for any $s>0, \varphi \in H^{s}$, we have

$$
\begin{aligned}
& \left\|G_{r}^{S, I, N} \varphi\right\|_{H^{s}} \leq C\|\varphi\|_{H^{s} \sup }\|K(., y)\|_{H^{s}}, \\
& \left\|G_{r}^{I, N} \varphi\right\|_{H^{s}} \leq C\|\varphi\|_{H^{s} \sup ^{y}}\|K(., y)\|_{H^{s}}, \\
& \left\|G_{r}^{S} \varphi\right\|_{H^{s}} \leq C\|\varphi\|_{H^{s} \sup _{x}}\left\|\frac{K(x, .)}{\int_{\mathbb{T}^{2}} K\left(x^{\prime}, .\right) \mu_{r}\left(d x^{\prime}\right)}\right\|_{H^{s}}^{2}
\end{aligned}
$$

Proof. Proof of (6.14). We first recall that $\int_{\mathbb{T}^{2}} K(x, y) f(t, x) d x$ is lower bounded by a positive constant $C$ independently of $y \in \mathbb{T}^{2}$ and $\left|\frac{\int_{\mathbb{T}^{2}} K(x, y) \varphi(x) \mu_{r}^{S, N}(d x)}{\int_{\mathbb{T}^{2}} K(u, y) \mu_{r}^{N}(d u)}\right| \leq\|\varphi\|_{\infty}$. 
Now we have

$$
\begin{aligned}
& \left\|G_{r}^{S, I, N} \varphi\right\|_{H^{s}}^{2}=\left\|\left(\mu_{r}^{I, N}, K \frac{\left(\mu_{r}^{S, N}, K \varphi\right)}{\left(\mu_{r}^{N}, K\right)\left(\mu_{r}, K\right)}\right)\right\|_{H^{s}}^{2} \\
& =\sum_{i, n_{1}, n_{2}}\left(1+\gamma \pi^{2}\left(n_{1}^{2}+n_{2}^{2}\right)\right)^{s}\left(\int_{\mathbb{T}^{2}} \int_{\mathbb{T}^{2}} K(z, y) \frac{\int_{\mathbb{T}^{2}} K(x, y) \varphi(x) \mu_{r}^{S, N}(d x)}{\int_{\mathbb{T}^{2}} K(u, y) \mu_{r}^{N}(d u) \int_{\mathbb{T}^{2}} K(u, y) \mu_{r}(d u)}\right. \\
& \leq \sum_{i, n_{1}, n_{2}}\left(1+\gamma \pi^{2}\left(n_{1}^{2}+n_{2}^{2}\right)\right)^{s} \int_{\mathbb{T}^{2}}^{i}\left(\frac{\left.\int_{\mathbb{T}^{2}, n_{2}}(z) \mu_{r}^{I, N}(d y) d z\right)^{2}}{\int_{\mathbb{T}^{2}} K(u, y) \mu_{r}^{N}(d u) \int_{\mathbb{T}^{2}} K(u, y) \mu_{r}(d u)}\right)^{2} \mu_{r}^{I, N}(d y) \\
& \leq C\|\varphi\|_{\infty}^{2} \int_{\mathbb{T}^{2}} \sum_{i, n_{1}, n_{2}}\left(1+\gamma \pi_{\mathbb{T}^{2}}\left(\int_{\mathbb{T}^{2}} K(z, y) f_{n_{1}, n_{2}}^{i}(z) d z\right)^{2} \mu_{r}^{I, N}(d y)\right. \\
& \leq C\|\varphi\|_{H^{s}}^{2} \sup _{y}\|K(., y)\|_{H^{s}}^{2} .
\end{aligned}
$$

Proof of (6.15).

$\left\|G_{r}^{I, N} \varphi\right\|_{H^{s}}=\left\|\varphi\left(\mu_{r}^{I, N}, \frac{K}{\left(\mu_{r}, K\right)}\right)\right\|_{H^{s}} \leq C\|\varphi\|_{H^{s}}\left\|\left(\mu_{r}^{I, N}, \frac{K}{\left(\mu_{r}, K\right)}\right)\right\|_{H^{s}}$ and$$
\left\|\left(\mu_{r}^{I, N}, K\right)\right\|_{H^{s}}^{2}=\sum_{i, n_{1}, n_{2}}\left(1+\gamma \pi^{2}\left(n_{1}^{2}+n_{2}^{2}\right)\right)^{s}\left(\left(\mu_{r}^{I, N}, \frac{K}{\left(\mu_{r}, K\right)}\right), f_{n_{1}, n_{2}}^{i}\right)_{L^{2}}^{2}
$$$$
\begin{aligned}
& =\sum_{i, n_{1}, n_{2}}\left(1+\gamma \pi^{2}\left(n_{1}^{2}+n_{2}^{2}\right)\right)^{s}\left(\int_{\mathbb{T}_{2}} \int_{\mathbb{T}^{2}} \frac{K(x, y)}{\int_{\mathbb{T}^{2}} K(u, y) \mu_{r}(d u)} f_{n_{1}, n_{2}}^{i}(x) \mu_{r}^{I, N}(d y) d x\right)^{2} \\
& \leq \sum_{i, n_{1}, n_{2}}\left(1+\gamma \pi^{2}\left(n_{1}^{2}+n_{2}^{2}\right)\right)^{s} \int_{\mathbb{T}^{2}} \frac{1}{\left(\int_{\mathbb{T}^{2}} K(u, y) \mu_{r}(d u)\right)^{2}} \mu_{r}^{I, N}(d y) \\
& \quad \times \int_{\mathbb{T}^{2}}\left(\int_{\mathbb{T}^{2}} K(x, y) f_{n_{1}, n_{2}}^{i}(x) d x\right)^{2} \mu_{r}^{I, N}(d y) \\
& \leq C \int_{\mathbb{T}^{2}} \sum_{i, n_{1}, n_{2}}\left(1+\gamma \pi^{2}\left(n_{1}^{2}+n_{2}^{2}\right)\right)^{s}\left(\int_{\mathbb{T}^{2}} K(x, y) f_{n_{1}, n_{2}}^{i}(x) d x\right)^{2} \mu_{r}^{I, N}(d y) \\
& \leq C \sup _{y}\|K(., y)\|_{H^{s}} .
\end{aligned}
$$

Proof of (6.16). Since $\int_{\mathbb{T}^{2}} \varphi^{2}(x) \mu_{r}^{S}(d x) \leq \int_{\mathbb{T}^{2}} \varphi^{2}(x) d x$, we have

$$
\begin{aligned}
\left\|G_{r}^{S} \varphi\right\|_{H^{s}} & =\left\|\frac{\left(\mu_{r}^{S}, K \varphi\right)}{\left(\mu_{r}, K\right)}\right\|_{H^{s}}^{2}=\sum_{i, n_{1}, n_{2}}\left(1+\gamma \pi^{2}\left(n_{1}^{2}+n_{2}^{2}\right)\right)^{s}\left(\frac{\left(\mu_{r}^{S}, K \varphi\right)}{\left(\mu_{r}, K\right)}, f_{n_{1}, n_{2}}^{i}\right)_{L^{2}}^{2} \\
& =\sum_{i, n_{1}, n_{2}}\left(1+\gamma \pi^{2}\left(n_{1}^{2}+n_{2}^{2}\right)\right)^{s}\left(\int_{\mathbb{T}^{2}} \frac{\int_{\mathbb{T}^{2}} K(x, y) \varphi(x) \mu_{r}^{S}(d x)}{\int_{\mathbb{T}^{2}} K\left(x^{\prime}, y\right) \mu_{r}\left(d x^{\prime}\right)} f_{n_{1}, n_{2}}^{i}(y) d y\right)^{2} \\
\leq & \sum_{i, n_{1}, n_{2}}\left(1+\gamma \pi^{2}\left(n_{1}^{2}+n_{2}^{2}\right)\right)^{s} \int_{\mathbb{T}^{2}} \varphi^{2}(x) \mu_{r}^{S}(d x) \\
& \left.\times \int_{\mathbb{T}^{2}}\left(\int_{\mathbb{T}^{2}} \frac{K(x, y)}{\int_{\mathbb{T}^{2}} K\left(x^{\prime}, y\right) \mu_{r}\left(d x^{\prime}\right)} f_{n_{1}, n_{2}}^{i}(y)\right) d y\right)^{2} \mu_{r}^{S}(d x) \\
\leq & C\|\varphi\|_{L^{2}}^{2} \int_{\mathbb{T}^{2}} \sum_{i, n_{1}, n_{2}}\left(1+\gamma \pi^{2}\left(n_{1}^{2}+n_{2}^{2}\right)\right)^{s}\left(\int_{\mathbb{T}^{2}} \frac{K(x, y)}{\int_{\mathbb{T}^{2}} K\left(x^{\prime}, y\right) \mu_{r}\left(d x^{\prime}\right)} f_{n_{1}, n_{2}}^{i}(y) d y\right)^{2} \mu_{r}^{S}(d x) \\
\leq & C\|\varphi\|_{H^{s} \sup _{x}}^{2}\left\|\frac{K(x, .)}{\int_{\mathbb{T}^{2}} K\left(x^{\prime}, .\right) \mu_{r}\left(d x^{\prime}\right)}\right\|_{H^{s}}^{2}
\end{aligned}
$$

We have the following immediate consequence of Proposition 6.19. 
Corollary 6.20. There exists a constant $C>0$ such that for any $s>0, \forall \mathcal{V} \in H^{-s}$, we have

$$
\begin{aligned}
& \left\|\left(G_{r}^{S, I, N}\right)^{*} \mathcal{V}\right\|_{H^{-s}} \leq C \sup _{y}\|K(., y)\|_{H^{s}}\|\mathcal{V}\|_{H^{-s}}, \\
& \left\|\left(G_{r}^{I, N}\right)^{*} \mathcal{V}\right\|_{H^{-s}} \leq C \sup _{y}\|K(., y)\|_{H^{s}}\|\mathcal{V}\|_{H^{-s}}, \\
& \left\|\left(G_{r}^{S}\right)^{*} \mathcal{V}\right\|_{H^{-s}} \leq C \sup _{x}\left\|\frac{K(x, .)}{\int_{\mathbb{T}^{2}} K\left(x^{\prime}, .\right) \mu_{r}\left(d x^{\prime}\right)}\right\|_{H^{s}}\|\mathcal{V}\|_{H^{-s}} .
\end{aligned}
$$

Let us prove the following results which will be useful to prove the tightness of $\left(U^{N}\right)_{N \geq 1}$ and $\left(V^{N}\right)_{N \geq 1}$ in $D\left(\mathbb{R}_{+}, H^{-s}\right)$.

Lemma 6.21. The pair of processes $\left(U^{N}, V^{N}\right)$ satisfies $\forall 0 \leq u<t$,

$$
\begin{aligned}
U_{t}^{N}= & \Upsilon(t-u) U_{u}^{N}+\beta \int_{u}^{t} \Upsilon(t-r)\left(G_{r}^{S, I, N}\right)^{*} Z_{r}^{N} d r-\beta \int_{u}^{t} \Upsilon(t-r)\left(G_{r}^{I, N}\right)^{*} U_{r}^{N} d r \\
& -\beta \int_{u}^{t} \Upsilon(t-r)\left(G_{r}^{S}\right)^{*} V_{r}^{N} d r+\int_{u}^{t} \Upsilon(t-r) d \widetilde{M}_{r}^{N}, \\
V_{t}^{N}= & \Upsilon(t-u) V_{u}^{N}-\beta \int_{u}^{t} \Upsilon(t-r)\left(G_{r}^{S, I, N}\right)^{*} Z_{r}^{N} d r+\beta \int_{u}^{t} \Upsilon(t-r)\left(G_{r}^{I, N}\right)^{*} U_{r}^{N} d r \\
& +\int_{u}^{t} \Upsilon(t-r)\left[\beta\left(G_{r}^{I, N}\right)^{*}-\alpha\right] V_{r}^{N} d r+\int_{u}^{t} \Upsilon(t-r) d \widetilde{L}_{r}^{N} .
\end{aligned}
$$

Proof. Let us consider a function $\phi$ belonging to $C^{1,2}\left(\mathbb{R}_{+} \times \mathbb{T}^{2}\right)$. By the Itô formula applied to $\phi\left(t, X_{t}^{i}\right)$ and using a similar computation as in subsections 5.1 and 6.1 , we obtain for $0 \leq u<t$,

$$
\begin{aligned}
\left(U_{t}^{N}, \phi_{t}\right) & =\left(U_{u}^{N}, \phi_{u}\right)+\gamma \int_{u}^{t}\left(U_{r}^{N}, \triangle \phi_{r}\right) d r+\int_{u}^{t}\left(U_{r}^{N}, \frac{\partial \phi_{r}}{\partial r}\right) d r \\
& +\beta \int_{u}^{t}\left(Z_{r}^{N},\left(\mu_{r}^{I, N}, K \frac{\left(\mu_{r}^{S, N}, \phi_{r} K\right)}{\left(\mu_{r}^{N}, K\right)\left(\mu_{r}, K\right)}\right)\right) d r-\beta \int_{u}^{t}\left(U_{r}^{N}, \phi_{r}\left(\mu_{r}^{I, N}, \frac{K}{\left(\mu_{r}, K\right)}\right)\right) d r \\
& -\beta \int_{u}^{t}\left(V_{r}^{N}, \frac{\left(\mu_{r}^{S}, \phi_{r} K\right)}{\left(\mu_{r}, K\right)}\right) d r+\int_{u}^{t}\left(\phi_{r}, d \widetilde{M}_{r}^{N}\right) .
\end{aligned}
$$

Let $\varphi \in H^{s+1}$ and $0 \leq u<t$, consider for $r \in[u, t]$ the mapping $\psi_{r}(x)=\Upsilon(t-r) \varphi(x)$. We have that $\psi \cdot(\cdot) \in C^{1,2}\left([u, t] \times \mathbb{T}^{2}\right)$. Indeed:

- For any $r \in[u, t], \psi_{r}(\cdot) \in H^{s+1}\left(\mathbb{T}^{2}\right) \subset C^{2}\left(\mathbb{T}^{2}\right)$.

- $\forall x \in \mathbb{T}^{2}$, the map $r \in[u, t] \mapsto \psi_{r}^{\prime}(x)=-\gamma \triangle(\Upsilon(t-r) \varphi(x))$ is continuous since $\Upsilon(t)$ is a strongly continuous semi-group and $-\gamma \Delta(\Upsilon(t-r) \varphi(x))=\Upsilon(t-r)(-\gamma \triangle \varphi(x))$, (see Proposition 2.5). Thus replacing $\phi$ by $\psi$ in the above equation we obtain,

$$
\begin{aligned}
\left(U_{t}^{N}, \varphi\right)= & \left(U_{u}^{N}, \Upsilon(t-u) \varphi\right)+\beta \int_{u}^{t}\left(Z_{r}^{N},\left(\mu_{r}^{I, N}, K \frac{\left(\mu_{r}^{S, N}, \Upsilon(t-r) \varphi K\right)}{\left(\mu_{r}^{N}, K\right)\left(\mu_{r}, K\right)}\right)\right) d r \\
& -\beta \int_{u}^{t}\left(U_{r}^{N}, \Upsilon(t-r) \varphi\left(\mu_{r}^{I, N}, \frac{K}{\left(\mu_{r}, K\right)}\right)\right) d r-\beta \int_{u}^{t}\left(V_{r}^{N}, \frac{\left(\mu_{r}^{S}, \Upsilon(t-r) \varphi K\right)}{\left(\mu_{r}, K\right)}\right) d r \\
& +\int_{u}^{t}\left(\Upsilon(t-r) \varphi, d \widetilde{M}_{r}^{N}\right) .
\end{aligned}
$$

We obtain (6.18) by similar argument.

Proposition 6.22. There exists $C>0$ such that for any stopping times $\bar{\tau}<\infty$ a.s and $\theta>0$,

$$
\begin{aligned}
& \mathbb{E}\left(\left\|\int_{\bar{\tau}}^{\bar{\tau}+\theta} \Upsilon(\bar{\tau}+\theta-r) d \widetilde{M}_{r}^{N}\right\|_{H^{-s}}^{2}\right) \leq C \theta, \\
& \mathbb{E}\left(\left\|\int_{\bar{\tau}}^{\bar{\tau}+\theta} \Upsilon(\bar{\tau}+\theta-r) d \widetilde{L}_{r}^{N}\right\|_{H^{-s}}^{2}\right) \leq C \theta .
\end{aligned}
$$


Proof. Proof of (6.19). Let us recall that $A_{r}^{N}(\varphi)=\beta\left(\mu_{r}^{S, N}, \varphi^{2}\left(\mu_{r}^{I, N}, \frac{K}{\left(\mu_{r}^{N}, K\right)}\right)\right)+2 \gamma\left(\mu_{r}^{S, N},(\nabla \varphi)^{2}\right)$ and $\int_{\tau^{N}}^{\tau^{N}+\theta}\left(\Upsilon\left(\tau^{N}+\theta-r\right) \varphi, d \widetilde{M}_{r}^{N}\right)=\sqrt{\frac{2 \gamma}{N}} \sum_{i=1}^{N} \int_{\tau^{N}}^{\tau^{N}+\theta} 1_{\left\{E_{r}^{i}=S\right\}} \nabla \Upsilon\left(\tau^{N}+\theta-r\right) \varphi\left(X_{r}^{i}\right) d B_{r}^{i}$

$$
-\sqrt{\frac{1}{N}} \sum_{i=1}^{N} \int_{\tau^{N}}^{\tau^{N}+\theta} \int_{0}^{\infty} 1_{\left\{E_{r^{-}}^{i}=S\right\}} \Upsilon\left(\tau^{N}+\theta-r\right) \varphi\left(X_{r}^{i}\right) 1_{\{u \leq \beta} \sum_{j=1}^{N} \frac{K\left(X_{r}^{i}, X_{r}^{j}\right)}{\left.\sum_{l=1}^{N} K\left(X_{r}^{l}, X_{r}^{j}\right)\right)} 1_{\left\{E_{r}^{j}=I\right\}} \bar{M}^{i}(d r, d u) .
$$

Now from Lemma 8.1 below, we have

$$
\begin{aligned}
\mathbb{E}\left(\left\|\int_{\bar{\tau}}^{\bar{\tau}+\theta} \Upsilon(\bar{\tau}+\theta-r) d \widetilde{M}_{r}^{N}\right\|_{H^{-s}}^{2}\right) & \leq \sum_{i, n_{1}, n_{2}} \mathbb{E}\left(\left(\int_{\bar{\tau}}^{\bar{\tau}+\theta} \Upsilon(\bar{\tau}+\theta-r) \rho_{n_{1}, n_{2}}^{i, s}, d \widetilde{M}_{r}^{N}\right)^{2}\right) \\
& =\sum_{i, n_{1}, n_{2}} \mathbb{E}\left(\int_{0}^{\theta} A_{r+\bar{\tau}}^{N}\left(\Upsilon(\theta-r) \rho_{n_{1}, n_{2}}^{i, s}\right) d r\right) \\
& =\sum_{i, n_{1}, n_{2}} \mathbb{E}\left(\int_{0}^{\theta} A_{r+\bar{\tau}}^{N}\left(e^{-(\theta-r) \lambda_{n_{1}, n_{2}}} \rho_{n_{1}, n_{2}}^{i, s}\right) d r\right) \\
& \leq \mathbb{E}\left(\int_{0}^{\theta} A_{r+\bar{\tau}}^{N}\left(\sum_{i, n_{1}, n_{2}} \rho_{n_{1}, n_{2}}^{i, s}\right) d r\right) \\
& \leq \theta C .
\end{aligned}
$$

A similar argument yields (6.20).

Proposition 6.23. For all $T>0$,

$$
\begin{aligned}
& \sup _{N \geq 10 \leq t \leq T} \sup _{0 \leq t} \mathbb{E}\left(\left\|U_{t}^{N}\right\|_{H^{-s}}^{2}\right)<\infty, \\
& \sup \sup _{N \geq 10 \leq t \leq T} \mathbb{E}\left(\left\|V_{t}^{N}\right\|_{H^{-s}}^{2}\right)<\infty .
\end{aligned}
$$

Proof. Choosing $u=0$ in equation (6.17) and (6.18), we get the estimates

$$
\begin{aligned}
\left\|U_{t}^{N}\right\|_{H^{-s}}^{2} \leq & 5\left\|\Upsilon(t) U_{0}^{N}\right\|_{H^{-s}}^{2}+5 \beta^{2} t \int_{0}^{t}\left\|\Upsilon(t-r)\left(G_{r}^{S, I, N}\right)^{*} Z_{r}^{N}\right\|_{H^{-s}}^{2} d r \\
& +5 \beta^{2} t \int_{0}^{t}\left\|\Upsilon(t-r)\left(G_{r}^{I, N}\right)^{*} U_{r}^{N}\right\|_{H^{-s}}^{2} d r+5 \beta^{2} t \int_{0}^{t}\left\|\Upsilon(t-r)\left(G_{r}^{S}\right)^{*} V_{r}^{N}\right\|_{H^{-s}}^{2} d r \\
& +5\left\|\int_{0}^{t} \Upsilon(t-r) d \widetilde{M_{r}^{N}}\right\|_{H^{-s}}^{2} \\
\left\|V_{t}^{N}\right\|_{H^{-s}}^{2} \leq & 6\left\|\Upsilon(t) V_{0}^{N}\right\|_{H^{-s}}^{2}+6 \beta^{2} t \int_{0}^{t}\left\|\Upsilon(t-r)\left(G_{r}^{S, I, N}\right)^{*} Z_{r}^{N}\right\|_{H^{-s}}^{2} d r \\
& +6 \beta^{2} t \int_{0}^{t}\left\|\Upsilon(t-r)\left(G_{r}^{I, N}\right)^{*} U_{r}^{N}\right\|_{H^{-s}}^{2} d r+6 t \beta^{2} \int_{0}^{t}\left\|\Upsilon(t-r) G_{r}^{S} V_{r}^{N}\right\|_{H^{-s}}^{2} d r \\
& +6 t \alpha^{2} \int_{0}^{t}\left\|V_{r}^{N}\right\|_{H^{-s}}^{2} d r+6\left\|\int_{0}^{t} \Upsilon(t-r) d \widetilde{L}_{r}^{N}\right\|_{H^{-s}}^{2} .
\end{aligned}
$$

From Corollary 6.20, we have

$$
\begin{aligned}
& \left\|U_{t}^{N}\right\|_{H^{-s}}^{2} \leq 5\left\|U_{0}^{N}\right\|_{H^{-s}}^{2}+5 t \beta^{2} C \sup _{x}\|K(x, .)\|_{H^{s}}^{2} \int_{0}^{t}\left\|U_{r}^{N}\right\|_{H^{-s}} d r \\
& \quad+5 C t \beta^{2} \sup _{x}\left\|\frac{K(x, .)}{\int_{\mathbb{T}^{2}} K\left(x^{\prime}, .\right) \mu_{r}\left(d x^{\prime}\right)}\right\|_{H^{s}}^{2} \int_{0}^{t}\left\|V_{r}^{N}\right\|_{H^{-s}}^{2} d r+5 t \beta^{2} C \sup _{x}\|K(x, .)\|_{H^{s}}^{2} \int_{0}^{t}\left\|Z_{r}^{N}\right\|_{H^{-s}}^{2} d r \\
& \quad+\left\|\int_{0}^{t} \Upsilon(t-r) d \widetilde{M}_{r}^{N}\right\|_{H^{-s}}^{2},
\end{aligned}
$$




$$
\begin{aligned}
\left\|V_{t}^{N}\right\|_{H^{-s}}^{2} \leq & 6\left\|V_{0}^{N}\right\|_{H^{-s}}^{2}+6 t \beta^{2} C \sup _{x}\|K(x, .)\|_{H^{s}}^{2} \int_{0}^{t}\left\|U_{r}^{N}\right\|_{H^{-s}} d r \\
& +6 t\left(\beta^{2} C \sup _{x}\left\|\frac{K(x, .)}{\int_{\mathbb{T}^{2}} K\left(x^{\prime}, .\right) \mu_{r}\left(d x^{\prime}\right)}\right\|_{H^{s}}^{2}+\alpha^{2}\right) \int_{0}^{t}\left\|V_{r}^{N}\right\|_{H^{-s}}^{2} d r \\
& +6 t \beta^{2} C \sup _{x}\|K(x, .)\|_{H^{s}}^{2} \int_{0}^{t}\left\|Z_{r}^{N}\right\|_{H^{-s}}^{2} d r+\left\|\int_{0}^{t} \Upsilon(t-r) d \widetilde{L}_{r}^{N}\right\|_{H^{-s}}^{2}
\end{aligned}
$$

Thus from Lemmas 8.2 in the Appendix below, 6.2 and Proposition 6.22, we have

$$
\begin{aligned}
\sup _{0 \leq t \leq T} \mathbb{E}\left(\left\|U_{t}^{N}\right\|_{H^{-s}}^{2}\right) \leq & 5 \mathbb{E}\left(\left\|U_{0}^{N}\right\|_{H^{-s}}^{2}\right)+5 T \beta^{2} C \int_{0}^{T}\left\{\sup _{0 \leq r \leq t} \mathbb{E}\left(\left\|U_{r}^{N}\right\|_{H^{-s}}^{2}\right)+\sup _{0 \leq r \leq t} \mathbb{E}\left(\left\|V_{r}^{N}\right\|_{H^{-s}}^{2}\right)\right\} d t \\
& +5 T^{2} \beta^{2} C \sup _{0 \leq t \leq T} \mathbb{E}\left(\left\|Z_{t}^{N}\right\|_{H^{-s}}\right)+C T \\
\sup _{0 \leq t \leq T} \mathbb{E}\left(\left\|V_{t}^{N}\right\|_{H^{-s}}^{2}\right) \leq & 6 \mathbb{E}\left(\left\|V_{0}^{N}\right\|_{H^{-s}}^{2}\right)+6 T\left(\beta^{2} C+\alpha^{2}\right) \int_{0}^{T}\left\{\sup _{0 \leq r \leq t} \mathbb{E}\left(\left\|U_{r}^{N}\right\|_{H^{-s}}^{2}\right)+\sup _{0 \leq r \leq t} \mathbb{E}\left(\left\|V_{r}^{N}\right\|_{H^{-s}}^{2}\right)\right\} d t \\
& +6 T^{2} \beta^{2} C \sup _{0 \leq t \leq T} \mathbb{E}\left(\left\|Z_{t}^{N}\right\|_{H^{-s}}^{2}\right)+C T .
\end{aligned}
$$

Hence summing (6.21) and (6.22) and applying Gronwall's lemma we deduce the result from Proposition 4.1 in section 4 and Proposition 6.10.

Now we can establish the tightness of the sequences $\left(U^{N}\right)_{N}$ and $\left(V^{N}\right)_{N}$.

Proposition 6.24. Both sequences of processes $U^{N}$ and $V^{N}$ are tight in $D\left(\mathbb{R}_{+}, H^{-s}\right)$.

Proof. We only establish the tighness of $U^{N}$ by showing that the conditions of the Proposition 6.7 are satisfied.

- (T1) is obtained by using the Proposition 6.23 and applying an argument similar to that of the proof of (T1) in Theorem 6.6.

- Proof of (T2). Let $\mathrm{T}>0, \varepsilon_{1}, \varepsilon_{2}>0,\left(\tau^{N}\right)_{N}$ a family of stopping times with $\tau^{N} \leq T$.

From equation (6.17), we have

$$
\begin{aligned}
U_{\tau^{N}+\theta}^{N}-U_{\tau^{N}}^{N} & =\left(\Upsilon(\theta)-I_{d}\right) U_{\tau^{N}}^{N}+\beta \int_{\tau^{N}}^{\tau^{N}+\theta} \Upsilon\left(\tau^{N}+\theta-r\right)\left(G_{r}^{S, I, N}\right)^{*} Z_{r}^{N} d r \\
& -\beta \int_{\tau^{N}}^{\tau^{N}+\theta} \Upsilon\left(\tau^{N}+\theta-r\right)\left(G_{r}^{I, N}\right)^{*} U_{r}^{N} d r-\beta \int_{\tau^{N}}^{\tau^{N}+\theta} \Upsilon\left(\tau^{N}+\theta-r\right)\left(G_{r}^{S}\right)^{*} V_{r}^{N} d r \\
& +\int_{\tau^{N}}^{\tau^{N}+\theta} \Upsilon\left(\tau^{N}+\theta-r\right) d \widetilde{M}_{r}^{N} \\
= & \left(\Upsilon(\theta)-I_{d}\right) U_{\tau^{N}}^{N}+\beta \int_{\tau^{N}}^{\tau^{N}+\theta} \Upsilon\left(\tau^{N}+\theta-r\right) J_{r}^{S, I, N}\left(Z^{N}, U^{N}, V^{N}\right) d r \\
& +\int_{\tau^{N}}^{\tau^{N}+\theta} \Upsilon\left(\tau^{N}+\theta-r\right) d \widetilde{M}_{r}^{N}
\end{aligned}
$$

where $J_{r}^{S, I, N}\left(Z^{N}, U^{N}, V^{N}\right)=\left(G_{r}^{S, I, N}\right)^{*} Z_{r}^{N}-\left(G_{r}^{I, N}\right)^{*} U_{r}^{N}-\left(G_{r}^{S}\right)^{*} V_{r}^{N}$.

We find $\delta>0$ and $N_{0} \geq 1$ such that

$$
\begin{aligned}
& \sup _{N \geq N_{0} \delta \geq \theta} \operatorname{Pup}\left(\left\|\left(\Upsilon(\theta)-I_{d}\right) U_{\tau^{N}}^{N}\right\|_{H^{-s}} \geq \varepsilon_{1}\right) \leq \varepsilon_{2}, \\
& \sup _{N \geq N_{0} \delta \geq \theta} \sup \left(\left\|\beta \int_{\tau^{N}}^{\tau^{N}+\theta} \Upsilon\left(\tau^{N}+\theta-r\right) J_{r}^{S, I, N}\left(Z^{N}, U^{N}, V^{N}\right) d r\right\|_{H^{-s}} \geq \varepsilon_{1}\right) \leq \varepsilon_{2}, \\
& \sup _{N \geq N_{0} \delta \geq \theta}\left(\left\|\int_{\tau^{N}}^{\tau^{N}+\theta} \Upsilon\left(\tau^{N}+\theta-r\right) d \widetilde{M}_{r}^{N}\right\|_{H^{-s}} \geq \varepsilon_{1}\right) \leq \varepsilon_{2} .
\end{aligned}
$$


- (6.23) is proved by similar reasoning to that of the proof of (6.5) in Proposition 6.11.

- Proof of (6.24). Let $l \in \mathbb{R}_{+} \backslash[0,1]$, we find $\delta>0$ such that $\tau^{N}+\delta \leq l T$ and such that (6.24) is satisfied. Since $\forall \varphi \in H^{s},\left\|\Upsilon\left(\tau^{N}+\theta-r\right) \varphi\right\|_{H^{s}} \leq\|\varphi\|_{H^{s}}$, from Corollary 6.20, Lemmas 8.2 below, 6.2 and Propositions 6.10 and 6.23, we have

$$
\begin{aligned}
\mathbb{P}\left(\| \beta \int_{\tau^{N}}^{\tau^{N}+\theta} \Upsilon\left(\tau^{N}\right.\right. & \left.+\theta-r) J_{r}^{S, I, N}\left(Z^{N}, U^{N}, V^{N}\right) d r \|_{H^{-s}} \geq \varepsilon_{1}\right) \\
& \leq \frac{\beta^{2}}{\varepsilon_{1}^{2}} \mathbb{E}\left(\left\|\int_{\tau^{N}}^{\tau^{N}+\theta} \Upsilon\left(\tau^{N}+\theta-r\right) J_{r}^{S, I, N}\left(Z^{N}, U^{N}, V^{N}\right) d r\right\|_{H^{-s}}^{2}\right) \\
& \leq \frac{\beta^{2} \theta}{\varepsilon_{1}^{2}} \mathbb{E}\left(\int_{\tau^{N}}^{\tau^{N}+\theta}\left\|\Upsilon\left(\tau^{N}+\theta-r\right) J_{r}^{S, I, N}\left(Z^{N}, U^{N}, V^{N}\right)\right\|_{H^{-s}}^{2} d r\right) \\
& \leq \frac{\beta^{2} \theta C}{\varepsilon_{1}^{2}} \sup _{y}\|K(., y)\|_{H^{s}} \mathbb{E}\left(\int_{\tau^{N}}^{\tau^{N}+\theta}\left\{\left\|Z_{r}^{N}\right\|_{H^{-s}}^{2}+\left\|U_{r}^{N}\right\|_{H^{-s}}^{2}\right\} d r\right) \\
& +\frac{\beta^{2} \theta C}{\varepsilon_{1}^{2}} \sup _{x}\left\|\frac{K(x, .)}{\int_{\mathbb{T}^{2}} K\left(x^{\prime}, .\right) \mu_{r}\left(d x^{\prime}\right)}\right\| \|_{H^{s}}^{\mathbb{E}}\left(\int_{\tau^{N}}^{\tau^{N}+\theta}\left\|V_{r}^{N}\right\|_{H^{-s}}^{2} d r\right) \\
& \leq \frac{\beta^{2} \delta^{2} C}{\varepsilon_{1}^{2}} \sup _{N \geq 10 \leq t \leq l T} \sup _{0} \mathbb{E}\left(\left\{\left\|Z_{t}^{N}\right\|_{H^{-s}}^{2}+\left\|U_{t}^{N}\right\|_{H^{-s}}^{2}+\left\|V_{t}^{N}\right\|_{H^{-s}}^{2}\right\}\right) \\
& \leq \frac{\beta^{2} \delta^{2} C}{\varepsilon_{1}^{2}} .
\end{aligned}
$$

So (6.24) is proved.

- Proof of (6.25). From result (6.19) in Proposition 6.22 we have,

$$
\begin{aligned}
\mathbb{P}\left(\left\|\int_{\tau^{N}}^{\tau^{N}+\theta} \Upsilon\left(\tau^{N}+\theta-r\right) d \widetilde{M}_{r}^{N}\right\|_{H^{-s}} \geq \varepsilon_{1}\right) & \leq \frac{1}{\varepsilon_{1}^{2}} \mathbb{E}\left(\left\|\int_{\tau^{N}}^{\tau^{N}+\theta} \Upsilon\left(\tau^{N}+\theta-r\right) d \widetilde{M}_{r}^{N}\right\|_{H^{-s}}^{2}\right) \\
& \leq \frac{1}{\varepsilon_{1}^{2}} \delta C,
\end{aligned}
$$

hence (6.25) is proved.

To show that all converging subsequences of $\left(U^{N}, V^{N}\right)_{N \geq 1}$ have the same limit we will need the next two Lemmas.

Lemma 6.25. For any $t \geq 0, \varphi \in H^{3}\left(\mathbb{T}^{2}\right)$, as $N \longrightarrow \infty$,

$$
\int_{0}^{t} \mathbb{E}\left(\left\|\left[G_{r}^{I, N}-G_{r}^{I}\right] \Upsilon(t-r) \varphi\right\|_{H^{s}}^{2}\right)^{\frac{1}{2}} d t \longrightarrow 0
$$

Proof. Since $s>3, H^{3} \hookrightarrow H^{s}$, thus

$$
\int_{0}^{t} \mathbb{E}\left(\left\|\left[G_{r}^{I, N}-G_{r}^{I}\right] \Upsilon(t-r) \varphi\right\|_{H^{s}}^{2}\right)^{\frac{1}{2}} d t \leq C \int_{0}^{t} \mathbb{E}\left(\left\|\left[G_{r}^{I, N}-G_{r}^{I}\right] \Upsilon(t-r) \varphi\right\|_{H^{3}}^{2}\right)^{\frac{1}{2}} d t
$$

Furtemore as $H^{3}$ is a Banach algebra (see Proposition 2.4) and $\|\Upsilon(t) \varphi\|_{H^{3}} \leq C\|\varphi\|_{H^{3}}$,

$$
\begin{aligned}
\left\|\left[G_{r}^{I, N}-G_{r}^{I}\right] \Upsilon(t-r) \varphi\right\|_{H^{3}} & =\left\|\Upsilon(t-r) \varphi\left(\mu_{r}^{I, N}-\mu_{r}^{I}, \frac{K}{\left(\mu_{r}, K\right)}\right)\right\|_{H^{3}} \\
& \leq C\|\varphi\|_{H^{3}}\left\|\left(\mu_{r}^{I, N}-\mu_{r}^{I}, \frac{K}{\left(\mu_{r}, K\right)}\right)\right\|_{H^{3}} .
\end{aligned}
$$

If we let $\operatorname{support}\{K(x, \cdot)\}=A(x)$, we will have 


$$
\left\|\left(\mu_{r}^{I, N}-\mu_{r}^{I}, \frac{K}{\left(\mu_{r}, K\right)}\right)\right\|_{H^{3}}^{2}=\sum_{|\eta| \leq 3} \int_{\mathbb{T}^{2}}\left|\int_{A(x)} \frac{D^{\eta} K(x, y)}{\int_{\mathbb{T}^{2}} K\left(x^{\prime}, y\right) \mu_{r}\left(d x^{\prime}\right)}\left(\mu_{r}^{I, N}-\mu_{r}^{I}\right)(d y)\right|^{2} d x
$$

furthemore from Remark 6.1 the map $y \in A(x) \mapsto D^{\eta} K(x, y)$ is continuous and bounded by $C \max _{0 \leq|\eta| \leq 3}\left\|k^{(|\eta|)}\right\|_{\infty}$. Thus as the map $y \in \mathbb{T}^{2} \mapsto \int_{\mathbb{T}^{2}} K\left(x^{\prime}, y\right) \mu_{r}\left(d x^{\prime}\right)$ is also continuous and lower bounded by a positive constant, the map $y \in A(x) \mapsto \frac{D^{\eta} K(x, y)}{\int_{\mathbb{T}^{2}} K\left(x^{\prime}, y\right) \mu_{r}\left(d x^{\prime}\right)}$ is continuous and bounded by $C \max _{0 \leq|\eta| \leq 3}\left\|k^{(|\eta|)}\right\|_{\infty}$. So we deduce from Theorem 5.6 that

$$
\left|\int_{A(x)} \frac{D^{\eta} K(x, y)}{\int_{\mathbb{T}^{2}} K\left(x^{\prime}, y\right) \mu_{r}\left(d x^{\prime}\right)}\left(\mu_{r}^{I, N}-\mu_{r}^{I}\right)(d y)\right|^{2} \stackrel{P}{\rightarrow} 0 .
$$

According to Lebesgue's dominated convergence theorem, $\mathbb{E}\left(\left\|\left(\mu_{r}^{I, N}-\mu_{r}^{I}, \frac{K}{\left(\mu_{r}, K\right)}\right)\right\|_{H^{3}}^{2}\right) \longrightarrow 0$, as $N \longrightarrow \infty$. Hence

$$
\begin{aligned}
\int_{0}^{t} \mathbb{E}\left(\|\left[G_{r}^{I, N}-\right.\right. & \left.\left.G_{r}^{I}\right] \Upsilon(t-r) \varphi \|_{H^{3}}^{2}\right)^{\frac{1}{2}} d t \\
& \leq C\|\varphi\|_{H^{3}} \int_{0}^{t} \mathbb{E}\left(\left\|\left(\mu_{r}^{I, N}-\mu_{r}^{I}, \frac{K}{\left(\mu_{r}, K\right)}\right)\right\|_{H^{3}}^{2}\right)^{\frac{1}{2}} d r \\
& \longrightarrow 0, \text { as } N \longrightarrow \infty .
\end{aligned}
$$

Hence the result.

Lemma 6.26. For any $t \geq 0, \varphi \in H^{s}\left(\mathbb{T}^{2}\right)$, as $N \longrightarrow \infty$,

$$
\int_{0}^{t} \mathbb{E}\left(\left\|\left[G_{r}^{S, I, N}-G_{r}^{S, I}\right] \Upsilon(t-r) \varphi\right\|_{H^{s}}^{2}\right)^{\frac{1}{2}} d t \longrightarrow 0
$$

Proof. We have

$$
\begin{aligned}
G_{r}^{S, I, N}( & \Upsilon(t-r) \varphi)(x)-G_{r}^{S, I}(\Upsilon(t-r) \varphi)(x) \\
& =\left(\mu_{r}^{I, N}, K(x, .) \frac{\left(\mu_{r}^{S, N}, K \Upsilon(t-r) \varphi\right)}{\left(\mu_{r}^{N}, K\right)\left(\mu_{r}, K\right)}\right)-\left(\mu_{r}^{I}, K(x, .) \frac{\left(\mu_{r}^{S}, K \Upsilon(t-r) \varphi\right)}{\left(\mu_{r}, K\right)\left(\mu_{r}, K\right)}\right) \\
& =\left(\mu_{r}^{I, N}-\mu_{r}^{I}, K(x, .) \frac{\left(\mu_{r}^{S}, K \Upsilon(t-r) \varphi\right)}{\left(\mu_{r}, K\right)^{2}}\right)+\left(\mu_{r}^{I, N}, K(x, .) \frac{\left(\mu_{r}^{S, N}-\mu_{r}^{S}, K \Upsilon(t-r) \varphi\right)}{\left(\mu_{r}, K\right)^{2}}\right) \\
& -\left(\mu_{r}^{I, N}, K(x, .) \frac{\left(\mu_{r}^{S, N}, K \Upsilon(t-r) \varphi\right)}{\left(\mu_{r}^{N}, K\right)\left(\mu_{r}, K\right)^{2}}\left(\mu_{r}^{N}-\mu_{r}, K\right)\right) .
\end{aligned}
$$

Furthemore:

a) Since :

$-\left|\frac{\int_{\mathbb{T}^{2}} K\left(x^{\prime}, y\right) \Upsilon(t-r) \varphi\left(x^{\prime}\right) \mu_{r}^{S}\left(d x^{\prime}\right)}{\int_{\mathbb{T}^{2}} K\left(x^{\prime \prime}, y\right) \mu_{r}\left(d x^{\prime \prime}\right)}\right| \leq\|\varphi\|_{\infty}$

- The map $y \in \mathbb{T}^{2} \mapsto \int_{\mathbb{T}^{2}} K\left(x^{\prime}, y\right) \mu_{r}\left(d x^{\prime}\right)$ is continuous and lower bounded by a positive constant.

- Under (H2), The map $y \in \mathbb{T}^{2} \mapsto \int_{\mathbb{T}^{2}} K\left(x^{\prime}, y\right) \Upsilon(t-r) \varphi\left(x^{\prime}\right) \mu_{r}^{S}\left(d x^{\prime}\right)$ is continuous

- From Remark 6.1, for any $x \in \mathbb{T}^{2},|\eta| \leq 3$, the map $y \in A(x) \mapsto D^{\eta} K(x, y)$ is continuous and bounded by $C \max _{0 \leq|\eta| \leq 3}\left\|k^{(|\eta|)}\right\|_{\infty}$,

the map $y \in A(x) \mapsto D^{\eta} K(x, y) \times \frac{\int_{\mathbb{T}^{2}} K\left(x^{\prime}, y\right) \Upsilon(t-r) \varphi\left(x^{\prime}\right) \mu_{r}^{S}\left(d x^{\prime}\right)}{\left(\int_{\mathbb{T}^{2}} K\left(x^{\prime \prime}, y\right) \mu_{r}\left(d x^{\prime \prime}\right)\right)^{2}}$ is continous and bounded by $C\|\varphi\|_{\infty} \max _{0 \leq|\eta| \leq 3}\left\|k^{(|\eta|)}\right\|_{\infty}$, hence 


$$
\left|\int_{A(x)} D^{\eta} K(x, y) \times \frac{\int_{\mathbb{T}^{2}} K\left(x^{\prime}, y\right) \Upsilon(t-r) \varphi\left(x^{\prime}\right) \mu_{r}^{S}\left(d x^{\prime}\right)}{\left(\int_{\mathbb{T}^{2}} K\left(x^{\prime \prime}, y\right) \mu_{r}\left(d x^{\prime \prime}\right)\right)^{2}}\left(\mu_{r}^{I, N}-\mu_{r}^{I}\right)(d y)\right|^{2} \stackrel{P}{\rightarrow} 0 .
$$

Furthemore, according to Lebesgue's dominated convergence theorem, we have

$$
\begin{aligned}
\int_{0}^{t} \mathbb{E}(\| & \left.\left(\mu_{r}^{I, N}-\mu_{r}^{I}, K \frac{\left(\mu_{r}^{S}, K \Upsilon(t-r) \varphi\right)}{\left(\mu_{r}, K\right)^{2}}\right) \|_{H^{s}}^{2}\right)^{\frac{1}{2}} d r \\
\leq & C \int_{0}^{t} \mathbb{E}\left(\left\|\left(\mu_{r}^{I, N}-\mu_{r}^{I}, K \frac{\left(\mu_{r}^{S}, K \Upsilon(t-r) \varphi\right)}{\left(\mu_{r}, K\right)^{2}}\right)\right\|_{H^{3}}^{2}\right)^{\frac{1}{2}} d r \\
\leq & C \int_{0}^{t}\left\{\sum _ { | \eta | \leq 3 } \int _ { \mathbb { T } ^ { 2 } } \mathbb { E } \left(\mid \int_{A(x)} D^{\eta} K(x, y) \times\right.\right. \\
& \left.\left.\times\left.\frac{\int_{\mathbb{T}^{2}} K\left(x^{\prime}, y\right) \Upsilon(t-r) \varphi\left(x^{\prime}\right) \mu_{r}^{S}\left(d x^{\prime}\right)}{\left(\int_{\mathbb{T}^{2}} K\left(x^{\prime \prime}, y\right) \mu_{r}\left(d x^{\prime \prime}\right)\right)^{2}}\left(\mu_{r}^{I, N}-\mu_{r}^{I}\right)(d y)\right|^{2}\right) d x\right\}^{\frac{1}{2}} d r \\
& \longrightarrow 0, \text { as } N \longrightarrow \infty .
\end{aligned}
$$

b) Let us prove that $\lim _{N \longrightarrow \infty} \int_{0}^{t} \mathbb{E}\left(\left\|\left(\mu_{r}^{I, N}, K \frac{\left(\mu_{r}^{S, N}-\mu_{r}^{S}, K \Upsilon(t-r) \varphi\right)}{\left(\mu_{r}, K\right)^{2}}\right)\right\|_{H^{s}}^{2}\right)^{\frac{1}{2}} d t=0$.

Under (H2):

$-\forall y \in \mathbb{T}^{2}$, the map $x \in \mathbb{T}^{2} \mapsto K(x, y) \Upsilon(t-r) \varphi(x)$ is Lipschitz and bounded and its $\|\cdot\|_{\infty}$ and $\|\cdot\|_{L}$ norms are bounded by $C\|k\|_{\infty}\|\varphi\|_{\infty}$ and $C\left(\|k\|_{\infty}+\|\varphi\|_{\infty}\right)$ respectively.

- For any $x \in \mathbb{T}^{2},|\eta| \leq 3$, the map $y \in A(x) \mapsto D^{\eta} K(x, y)$ is continuous and bounded by $C \max _{0 \leq|\eta| \leq 3}\left\|k^{(|\eta|)}\right\|_{\infty}$.

$-\forall y \in \mathbb{T}^{2}, \frac{1}{\left(\mu_{r}, K(., y)\right)}$ is bounded by a positive contant independent of $y$.

Thus

$$
\begin{aligned}
& \left\|\left(\mu_{r}^{I, N}, K \frac{\left(\mu_{r}^{S, N}-\mu_{r}^{S}, K \Upsilon(t-r) \varphi\right)}{\left(\mu_{r}, K\right)^{2}}\right)\right\|_{H^{s}}^{2} \leq C\left\|\left(\mu_{r}^{I, N}, K \frac{\left(\mu_{r}^{S, N}-\mu_{r}^{S}, K \Upsilon(t-r) \varphi\right)}{\left(\mu_{r}, K\right)^{2}}\right)\right\|_{H^{3}}^{2} \\
& \quad=C \sum_{|\eta| \leq 3} \int_{\mathbb{T}^{2}}\left|\int_{A(x)} D^{\eta} K(x, y) \times \frac{\int_{\mathbb{T}^{2}} K\left(x^{\prime}, y\right) \Upsilon(t-r) \varphi\left(x^{\prime}\right)\left(\mu_{r}^{S, N}-\mu_{r}^{S}\right)\left(d x^{\prime}\right)}{\left(\int_{\mathbb{T}^{2}} K\left(x^{\prime \prime}, y\right) \mu_{r}\left(d x^{\prime \prime}\right)\right)^{2}} \mu_{r}^{I, N}(d y)\right|^{2} d x \\
& \quad \leq C \sum_{|\eta| \leq 3} \int_{\mathbb{T}^{2}} \int_{A(x)}\left|D^{\eta} K(x, y) \times \int_{\mathbb{T}^{2}} K\left(x^{\prime}, y\right) \Upsilon(t-r) \varphi\left(x^{\prime}\right)\left(\mu_{r}^{S, N}-\mu_{r}^{S}\right)\left(d x^{\prime}\right)\right|^{2} \mu_{r}^{I, N}(d y) d x \\
& \quad \leq C\left(\|k\|_{\infty},\|\varphi\|_{\infty}, \max _{0 \leq|\eta| \leq 3}\left\|k^{(|\eta|)}\right\|_{\infty}\right) d_{F}^{2}\left(\mu_{r}^{S, N}, \mu_{r}^{S}\right) .
\end{aligned}
$$

Hence since $d_{F}^{2}\left(\mu_{r}^{S, N}, \mu_{r}^{S}\right) \stackrel{P}{\rightarrow} 0$, and $d_{F}^{2}\left(\mu_{r}^{S, N}, \mu_{r}^{S}\right) \leq 4$, according to Lebesgue's dominated convergence theorem, we have

$$
\begin{aligned}
\int_{0}^{t} \mathbb{E}\left(\left\|\left(\mu_{r}^{I, N}, K \frac{\left(\mu_{r}^{S, N}-\mu_{r}^{S}, K \Upsilon(t-r) \varphi\right)}{\left(\mu_{r}, K\right)^{2}}\right)\right\|_{H^{s}}^{2}\right)^{\frac{1}{2}} d t \\
\leq C\left(\|\varphi\|_{\infty}, \max _{0 \leq|\eta| \leq 3}\left\|k^{(|\eta|)}\right\|_{\infty}\right) \int_{0}^{t} \mathbb{E}\left(d_{F}^{2}\left(\mu_{r}^{S, N}, \mu_{r}^{S}\right)\right)^{\frac{1}{2}} d r \\
\longrightarrow 0, \text { as } N \longrightarrow \infty .
\end{aligned}
$$

c) Finaly we show that $\lim _{N \longrightarrow \infty} \int_{0}^{t} \mathbb{E}\left(\left\|\left(\mu_{r}^{I, N}, K \frac{\left(\mu_{r}^{S, N}, K \Upsilon(t-r) \varphi\right)}{\left(\mu_{r}^{N}, K\right)\left(\mu_{r}, K\right)^{2}}\left(\mu_{r}^{N}-\mu_{r}, K\right)\right)\right\|_{H^{s}}^{2}\right)^{\frac{1}{2}} d t=0$.

Since for all $x \in \mathbb{T}^{2}, K(x,$.$) is Lipschitz and bounded by \|k\|_{\infty}$ and For any $x \in \mathbb{T}^{2},|\eta| \leq 3$, the map $y \in A(x) \mapsto D^{\eta} K(x, y)$ is bounded by $C \max _{0 \leq|\eta| \leq 3}\left\|k^{(|\eta|)}\right\|_{\infty}$, we have 


$$
\begin{aligned}
& \left\|\left(\mu_{r}^{I, N}, K \frac{\left(\mu_{r}^{S, N}, K \Upsilon(t-r) \varphi\right)}{\left(\mu_{r}^{N}, K\right)\left(\mu_{r}, K\right)^{2}}\left(\mu_{r}^{N}-\mu_{r}, K\right)\right)\right\|_{H^{s}}^{2} \\
& \quad \leq C\left\|\left(\mu_{r}^{I, N}, K \frac{\left(\mu_{r}^{S, N}, K \Upsilon(t-r) \varphi\right)}{\left(\mu_{r}^{N}, K\right)\left(\mu_{r}, K\right)^{2}}\left(\mu_{r}^{N}-\mu_{r}, K\right)\right)\right\|_{H^{3}}^{2} \\
& \quad \leq C \sum_{|\eta| \leq 3} \int_{\mathbb{T}^{2}}\left(\int_{A(x)}\left|D^{\eta} K(x, y)\right| \times\left|\frac{\int_{\mathbb{V}^{2}} \Upsilon(t-r) \varphi\left(x^{\prime}\right) K\left(x^{\prime}, y\right) \mu_{r}^{S, N}\left(d x^{\prime}\right)}{\int_{\mathbb{T}^{2}} K(u, y) \mu_{r}^{N}(d u)\left(\int_{\mathbb{T}^{2}} K(u, y) \mu_{r}(d u)\right)^{2}}\right|\right. \\
& \quad \leq C\|\varphi\|_{\infty}^{2} \sum_{|\eta| \leq 3} \int_{\mathbb{T}^{2}}\left(\int_{A(x)}\left|D^{\eta} K(x, y)\right| \times\left|\int_{\mathbb{T}^{2}} K(u, y)\left(\mu_{r}^{N}-\mu_{r}\right)(d u)\right| \mu_{r}^{I, N}(d y)\right)^{2} d x \\
& \quad \leq C\left(\|k\|_{\infty},\|\varphi\|_{\infty}, \max _{0 \leq|\eta| \leq 3}\left\|k^{(|\eta|)}\right\|_{\infty}\right) d_{F}^{2}\left(\mu_{r}^{N}, \mu_{r}\right) .
\end{aligned}
$$

Thus

$$
\begin{aligned}
& \int_{0}^{t} \mathbb{E}\left(\|\left(\mu_{r}^{I, N}, K\right.\right.\left.\left.\frac{\left(\mu_{r}^{S, N}, K \Upsilon(t-r) \varphi\right)}{\left(\mu_{r}^{N}, K\right)\left(\mu_{r}, K\right)^{2}}\left(\mu_{r}^{N}-\mu_{r}, K\right)\right) \|_{H^{s-1}}^{2}\right)^{\frac{1}{2}} d t \\
& \leq C\left(\|k\|_{\infty},\|\varphi\|_{\infty}, \max _{0 \leq|\eta| \leq 3}\left\|k^{(|\eta|)}\right\|_{\infty}\right) \int_{0}^{t} \mathbb{E}\left(d_{F}^{2}\left(\mu_{r}^{N}, \mu_{r}\right)\right)^{\frac{1}{2}} d r \\
& \quad \longrightarrow 0, \text { as } N \longrightarrow \infty .
\end{aligned}
$$

The next Proposition establish the evolution equations of all limit points $(U, V)$ of the sequence $\left(U^{N}, V^{N}\right)$.

Proposition 6.27. Any limit point $(U, V)$ of the sequence $\left(U^{N}, V^{N}\right)$ satisfies

$$
\begin{aligned}
U_{t}= & \Upsilon(t) U_{0}+\beta \int_{0}^{t} \Upsilon(t-r)\left(G_{r}^{S, I}\right)^{*} Z_{r} d r-\beta \int_{0}^{t} \Upsilon(t-r)\left(G_{r}^{I}\right)^{*} U_{r} d r-\beta \int_{0}^{t} \Upsilon(t-r)\left(G_{r}^{S}\right)^{*} V_{r} d r \\
& +\int_{0}^{t} \Upsilon(t-r) W_{r}^{1} \\
V_{t}= & \Upsilon(t) V_{0}-\beta \int_{0}^{t} \Upsilon(t-r)\left(G_{r}^{S, I}\right)^{*} Z_{r} d r+\beta \int_{0}^{t} \Upsilon(t-r)\left(G_{r}^{I}\right)^{*} U_{r} d r+\beta \int_{0}^{t} \Upsilon(t-r)\left(G_{r}^{S}\right)^{*} V_{r} d r \\
& -\alpha \int_{0}^{t} \Upsilon(t-r) V_{r} d r+\int_{0}^{t} \Upsilon(t-r) d W_{r}^{2} .
\end{aligned}
$$

Proof. We prove this Proposition by taking the weak limit in the equations (6.17) and (6.18). Note first that from Propositions 6.11 and 6.24 there exists a subsequence along which the sequences $\left(U^{N}, V^{N}\right)_{N}$, and $\left(Z^{N}\right)_{N}$ converge in law towards $(U, V)$ and $\mathrm{Z}$ respectively.

1- We first prove that $\int_{0}^{t}\left(U_{r}^{N}, G_{r}^{I, N} \Upsilon(t-r) \varphi\right) d r \stackrel{L}{\rightarrow} \int_{0}^{t}\left(U_{r}, G_{r}^{I} \Upsilon(t-r) \varphi\right) d r$.

We have $\int_{0}^{t}\left(U_{r}^{N}, G_{r}^{I, N} \Upsilon(t-r) \varphi\right) d r=\int_{0}^{t}\left(U_{r}^{N}, G_{r}^{I} \Upsilon(t-r) \varphi\right) d r$

- Since $G_{r}^{I} \Upsilon(t-r) \varphi \in H^{s}$,

$$
+\int_{0}^{t}\left(U_{r}^{N},\left[G_{r}^{I, N}-G_{r}^{I}\right] \Upsilon(t-r) \varphi\right) d r
$$

$$
\int_{0}^{t}\left(U_{r}^{N}, G_{r}^{I} \Upsilon(t-r) \varphi\right) d r \stackrel{L}{\rightarrow} \int_{0}^{t}\left(U_{r}, G_{r}^{I} \Upsilon(t-r) \varphi\right) d r
$$

- From Lemma 6.25 and Proposition $6.23 \int_{0}^{t}\left(U_{r}^{N},\left[G_{r}^{I, N}-G_{r}^{I}\right] \Upsilon(t-r) \varphi\right) d r \longrightarrow 0$ in $L^{1}(\mathbb{P})$. 
Indeed, $\mathbb{E}\left(\left|\int_{0}^{t}\left(U_{r}^{N},\left[G_{r}^{I, N}-G_{r}^{I}\right] \Upsilon(t-r) \varphi\right) d r\right|\right) \leq$

$$
\leq \sup _{N \geq 10 \leq r \leq t} \sup _{0 \leq r} \mathbb{E}\left(\left\|U_{r}^{N}\right\|_{H^{-s}}^{2}\right)^{\frac{1}{2}} \int_{0}^{t} \mathbb{E}\left(\left\|\left[G_{r}^{I, N}-G_{r}^{I}\right] \Upsilon(t-r) \varphi\right\|_{H^{s}}^{2}\right)^{\frac{1}{2}} d r .
$$

2- Since $V^{N}$ converges in law in $D\left(\mathbb{R}_{+}, H^{-s}\right)$ towards $V$,

$$
\int_{0}^{t}\left(\Upsilon(t-r)\left(G_{r}^{S}\right)^{*} V_{r}^{N}, \varphi\right) d r \stackrel{L}{\rightarrow} \int_{0}^{t}\left(\Upsilon(t-r)\left(G_{r}^{S}\right)^{*} V_{r}, \varphi\right) d r
$$

3- The convergence $\int_{0}^{t}\left(Z_{r}^{N}, G_{r}^{S, I, N} \Upsilon(t-r) \varphi\right) d r \stackrel{L}{\rightarrow} \int_{0}^{t}\left(Z_{r}, G_{r}^{S, I} \Upsilon(t-r) \varphi\right) d r$ follows from the same argument as the one in 1-, using this time Lemma 6.26 and Proposition 6.10.

4- Since for any $\varphi \in H^{s+1}, \Upsilon(t) \varphi \in H^{s+1}$, from Theorem 4.2 and Proposition 6.18, we have

$$
\left(U_{0}^{N}, \Upsilon(t) \varphi\right) \stackrel{L}{\rightarrow}\left(U_{0}, \Upsilon(t) \varphi\right) \text { and } \int_{0}^{t}\left(\Upsilon(t-r) \varphi, d \widetilde{M}_{r}^{N}\right) \stackrel{L}{\rightarrow} \int_{0}^{t}\left(\Upsilon(t-r) \varphi, d W_{r}^{1}\right)
$$

From Proposition 6.16 we deduce that all limit points $(U, V)$ of $\left(U^{N}, V^{N}\right)_{N \geq 1}$ are elements of $\left(C\left(\mathbb{R}_{+}, H^{-s}\right)\right)^{2}$, thus since it is so easy to see that equations (6.26) and (6.27) are the same as equations (6.10) and (6.11) repectively, we end the proof of Theorem 6.14 by showing that the system formed by the equations (6.26) and (6.27) admits a unique solution $(U, V) \in\left(C\left(\mathbb{R}_{+}, H^{-s}\right)\right)^{2}$.

Proposition 6.28. Suppose that $\left(U^{1}, V^{1}\right)$ and $\left(U^{2}, V^{2}\right)$ both belong to $\left(C\left(\mathbb{R}_{+}, H^{-s}\right)\right)^{2}$ and are solutions to equations (6.26) and (6.27) with $\left(U_{0}^{1}, V_{0}^{1}\right)=\left(U_{0}^{2}, V_{0}^{2}\right)$ then $\left(U^{1}, V^{1}\right)=\left(U^{2}, V^{2}\right)$

Proof. We have thus

$$
U_{t}^{1}-U_{t}^{2}=-\beta \int_{0}^{t} \Upsilon(t-r)\left(G_{r}^{I}\right)^{*}\left(U_{r}^{1}-U_{r}^{2}\right) d r-\beta \int_{0}^{t} \Upsilon(t-r)\left(G_{r}^{S}\right)^{*}\left(V_{r}^{1}-V_{r}^{2}\right) d r
$$

$$
\left\|U_{t}^{1}-U_{t}^{2}\right\|_{H^{-s}} \leq \beta \int_{0}^{t}\left\|\Upsilon(t-r)\left(G_{r}^{I}\right)^{*}\left(U_{r}^{1}-U_{r}^{2}\right)\right\|_{H^{-s}} d r+\beta \int_{0}^{t}\left\|\Upsilon(t-r)\left(G_{r}^{S}\right)^{*}\left(V_{r}^{1}-V_{r}^{2}\right)\right\|_{H^{-s}} d r .
$$

Moreover from Corollary 6.20, we deduce that

$$
\begin{aligned}
& \left\|U_{t}^{1}-U_{t}^{2}\right\|_{H^{-s}} \leq \beta C \sup _{y}\|K(., y)\|_{H^{s}} \int_{0}^{t}\left\|U_{r}^{1}-U_{r}^{2}\right\|_{H^{-s}} d r+ \\
& \quad \beta C \sup _{x}\left\|\frac{K(x, .)}{\int_{\mathbb{T}^{2}} K\left(x^{\prime}, .\right) \mu_{r}\left(d x^{\prime}\right)}\right\|_{H^{s}} \int_{0}^{t}\left\|V_{r}^{1}-V_{r}^{2}\right\|_{H^{-s}} d r,
\end{aligned}
$$

hence using Lemmas 8.2 below and 6.2, we obtain

On the other hand

$$
\left\|U_{t}^{1}-U_{t}^{2}\right\|_{H^{-s}} \leq \beta C \int_{0}^{t}\left\{\left\|U_{r}^{1}-U_{r}^{2}\right\|_{H^{-s}}+\left\|V_{r}^{1}-V_{r}^{2}\right\|_{H^{-s}}\right\} d r .
$$

hence

$$
V_{t}^{1}-V_{t}^{2}=-\left(U_{t}^{1}-U_{t}^{2}\right)-\alpha \int_{0}^{t} \Upsilon(t-r)\left(V_{r}^{1}-V_{r}^{2}\right) d r
$$

$$
\left\|V_{t}^{1}-V_{t}^{2}\right\|_{H^{-s}} \leq\left\|U_{t}^{1}-U_{t}^{2}\right\|_{H^{-s}}+\alpha \int_{0}^{t}\left\|V_{r}^{1}-V_{r}^{2}\right\|_{H^{-s}} d r .
$$

Summing (6.28) and (6.29) and applying Gronwall's lemma we obtain $\left(U^{1}, V^{1}\right)=\left(U^{2}, V^{2}\right)$.

The next two Lemmas will be useful to show an additional result about the regularity of the pair of Processes $(U, V)$.

Lemma 6.29. For any $x \in \mathbb{T}^{2}$, let us set $\mathcal{K}_{t}^{I}(x)=\left(\mu_{t}^{I}, \frac{K(x, .)}{\left(\mu_{t}, K\right)}\right)$. For any $t, t_{0} \in \mathbb{R}_{+}$, there exists $C>0$ such that

$$
\left\|\mathcal{K}_{t}^{I}(.)-\mathcal{K}_{t_{0}}^{I}(.)\right\|_{H^{2}} \leq C\left(d_{F}\left(\mu_{t}, \mu_{t_{0}}\right)+d_{F}\left(\mu_{t}^{I}, \mu_{t_{0}}^{I}\right)\right)
$$


Proof. Let $t, t_{0} \in \mathbb{R}_{+}$, we have

Furthemore:

$$
\mathcal{K}_{t}^{I}(x)-\mathcal{K}_{t_{0}}^{I}(x)=\left(\mu_{t}^{I}-\mu_{t_{0}}^{I}, \frac{K(x, .)}{\left(\mu_{t}, K\right)}\right)+\left(\mu_{t}^{I}, \frac{K(x, .)\left(\mu_{t}-\mu_{t_{0}}, K\right)}{\left(\mu_{t}, K\right)\left(\mu_{t_{0}}, K\right)}\right) .
$$

1- Since the map $y \in A(x) \mapsto \int_{\mathbb{T}^{2}} K\left(x^{\prime}, y\right) \mu_{r}\left(d x^{\prime}\right)$ is Lipschitz (with the Lipschitz constant $2 \sqrt{2} C_{k} \delta_{2}$ (see Lemma 5.7)) and lower bounded by a positive constant and from Remark 6.1, $\forall|\eta| \leq 2$ the map

$y \in A(x) \mapsto D^{\eta} K(x, y)$ is Lipschitz (with the Lipschitz constant independent of $x$ ) and bounded by $C \max _{0 \leq|\eta| \leq 2}\left\|k^{(|\eta|)}\right\|_{\infty}$, the map $y \in A(x) \mapsto \frac{D^{\eta} K(x, y)}{\int_{\mathbb{T}^{2}} K\left(x^{\prime}, y\right) \mu_{r}\left(d x^{\prime}\right)}$ is Lipschitz (with the constant Lipschitz independent of $x$ ) and bounded by $C_{|\eta| \leq 2}\left\|k^{(|\eta|)}\right\|_{\infty}$.

Hence $\left\|\left(\mu_{t}^{I}-\mu_{t_{0}}^{I}, \frac{K(x, .)}{\left(\mu_{t}, K\right)}\right)\right\|_{H^{2}}^{2}=\sum_{|\eta| \leq 2} \int_{\mathbb{V}^{2}}\left|\int_{A(x)} \frac{D^{\eta} K(x, y)}{\int_{\mathbb{T}^{2}} K\left(x^{\prime}, y\right) \mu_{t}\left(d x^{\prime}\right)}\left(\mu_{t}^{I}-\mu_{t_{0}}^{I}\right)(d y)\right|^{2} d x$

$$
\leq C d_{F}\left(\mu_{t}^{I}, \mu_{t_{0}}^{I}\right) \text {. }
$$

2- Since $K(x,$.$) is Lipschitz (with the Lipschit constant 2 \sqrt{2} C_{k}$ (see Lemma 5.7)) and bounded by $\|k\|_{\infty}$ and the map $y \in A(x) \mapsto \int_{\mathbb{T}^{2}} K\left(x^{\prime}, y\right) \mu_{r}\left(d x^{\prime}\right)$ is lower bounded and $\forall|\eta| \leq 2$ the map $y \in A(x) \mapsto D^{\eta} K(x, y)$ is bounded,

$$
\begin{aligned}
& \left\|\left(\mu_{t}^{I}, \frac{K(x, .)\left(\mu_{t}-\mu_{t_{0}}, K\right)}{\left(\mu_{t}, K\right)\left(\mu_{t_{0}}, K\right)}\right)\right\|_{H^{2}}^{2} \\
& =\sum_{|\eta| \leq 2} \int_{\mathbb{T}^{2}}\left|\int_{A(x)} \frac{D^{\eta} K(x, y)\left(\mu_{t}-\mu_{t_{0}}, K(., y)\right)}{\int_{\mathbb{T}^{2}} K\left(x^{\prime}, y\right) \mu_{t}\left(d x^{\prime}\right) \int_{\mathbb{T}^{2}} K\left(x^{\prime}, y\right) \mu_{t_{0}}\left(d x^{\prime}\right)} \mu_{t_{0}}^{I}(d y)\right|^{2} d x, \\
& \leq C \sum_{|\eta| \leq 2} \int_{\mathbb{T}^{2}} \int_{A(x)}\left|\frac{D^{\eta} K(x, y)\left(\mu_{t}-\mu_{t_{0}}, K(., y)\right)}{\int_{\mathbb{T}^{2}} K\left(x^{\prime}, y\right) \mu_{t}\left(d x^{\prime}\right) \int_{\mathbb{T}^{2}} K\left(x^{\prime}, y\right) \mu_{t_{0}}\left(d x^{\prime}\right)}\right|^{2} \mu_{t_{0}}^{I}(d y) d x, \\
& \leq C d_{F}^{2}\left(\mu_{t}, \mu_{t_{0}}\right) .
\end{aligned}
$$

So the result follows from $1-$ and 2 -.

Lemma 6.30. Let $\varphi \in H^{2}\left(\mathbb{T}^{2}\right)$, for any $x \in \mathbb{T}^{2}$, let us $\operatorname{set} \mathcal{K}_{t}^{S}(x, \varphi)=\frac{\left(\mu_{t}^{S}, \varphi K(x, .)\right)}{\left(\mu_{t}, K(x, .)\right)}$.

For any $t, t_{0} \in \mathbb{R}_{+}$,

$$
\left\|\mathcal{K}_{t}^{S}(., \varphi)-\mathcal{K}_{t_{0}}^{S}(., \varphi)\right\|_{H^{2}} \leq C\left(\|\varphi\|_{H^{2}}\right)\left(d_{F}\left(\mu_{t}, \mu_{t_{0}}\right)+d_{F}\left(\mu_{t}^{S}, \mu_{t_{0}}^{S}\right)\right) .
$$

Proof. Let $t, t_{0} \in \mathbb{R}_{+}$, we have

$\mathcal{K}_{t}^{S}(x, \varphi)-\mathcal{K}_{t_{0}}^{S}(x, \varphi)=\frac{\left(\mu_{t}^{S}-\mu_{t_{0}}^{S}, \varphi K(x, .)\right)}{\left(\mu_{t_{0}}, K(x, .)\right)}+\frac{\left(\mu_{t}^{S}, \varphi K(x, .)\right)}{\left(\mu_{t}, K(x, .)\right)\left(\mu_{t_{0}}, K(x, .)\right)}\left(\mu_{t}-\mu_{t_{0}}, K(x,).\right)$.

Furthemore:

1- Since from Remark 6.1, $\forall|\eta| \leq 2$, the map $y \in A(x) \mapsto \varphi(y) D^{\eta} K(x, y)$ is Lipschitz ( with the Lipschitz constant independent of $\mathrm{x}$ ) and bounded by $C\|\varphi\|_{\infty} \max _{0 \leq|\eta| \leq 2}\left\|k^{(|\eta|)}\right\|_{\infty}$ and $H^{2}$ is Banach algebra and an easy adaptation of the proof of Lemma 8.2 below yields that $\left\|\frac{1}{\left(\mu_{t_{0}}, K\right)}\right\|_{H^{2}}<C$,

$$
\begin{aligned}
\left\|\frac{\left(\mu_{t}^{S}-\mu_{t_{0}}^{S}, \varphi K\right)}{\left(\mu_{t_{0}}, K\right)}\right\|_{H^{2}}^{2} & \leq C\left\|\frac{1}{\left(\mu_{t_{0}}, K\right)}\right\|_{H^{2}}^{2}\left\|\left(\mu_{t}^{S}-\mu_{t_{0}}^{S}, \varphi K\right)\right\|_{H^{2}}^{2} \\
& =C \sum_{|\eta| \leq 2} \int_{\mathbb{T}^{2}}\left|\int_{A(x)} \varphi(y) D^{\eta} K(x, y)\left(\mu_{t}^{S}-\mu_{t_{0}}^{S}\right)(d y)\right|^{2} d x \\
& \leq C\left(\|\varphi\|_{\infty}\right) d_{F}^{2}\left(\mu_{t}^{S}, \mu_{t_{0}}^{S}\right) .
\end{aligned}
$$


2- Using an argument similar to the proof of (6.14) in proposition 6.19 and an easy adaptation of Lemma 8.2 below, we have

$$
\left\|\frac{\left(\mu_{t}^{S}, \varphi K\right)}{\left(\mu_{t}, K\right)\left(\mu_{t_{0}}, K\right)}\right\|_{H^{2}} \leq C\|\varphi\|_{H^{2} \operatorname{Sup}_{x}}\left\|\frac{K(x, .)}{\int_{\mathbb{T}^{2}} K\left(., x^{\prime \prime}\right) \mu_{t}\left(d x^{\prime \prime}\right) \int_{\mathbb{T}^{2} K\left(., x^{\prime}\right)} \mu_{t_{0}}\left(d x^{\prime}\right)}\right\|_{H^{2}} \leq C .
$$

Now since from Remark 6.1, $\forall|\eta| \leq 2$, the map $y \in A(x) \mapsto D^{\eta} K(x, y)$ is Lipschitz (with the Lipschitz constant independent of $\mathrm{x}$ ) and bounded by $C \max _{0 \leq|\eta| \leq 2}\left\|k^{(|\eta|)}\right\|_{\infty}$ and $H^{2}$ is Banach algebra, one has

$$
\begin{aligned}
\left\|\frac{\left(\mu_{t}^{S}, \varphi K\right)}{\left(\mu_{t}, K\right)\left(\mu_{t_{0}}, K\right)}\left(\mu_{t}-\mu_{t_{0}}, K\right)\right\|_{H^{2}}^{2} & \leq C\left\|\frac{\left(\mu_{t}^{S}, \varphi K\right)}{\left(\mu_{t}, K\right)\left(\mu_{t_{0}}, K\right)}\right\|_{H^{2}}^{2}\left\|\left(\mu_{t}-\mu_{t_{0}}, K\right)\right\|_{H^{2}}^{2} \\
& =C\left(\|\varphi\|_{H^{2}}\right) \sum_{|\eta| \leq 2} \int_{\mathbb{T}^{2}}\left|\int_{A(x)} D^{\eta} K(x, y)\left(\mu_{t}-\mu_{t_{0}}\right)(d y)\right|^{2} d x \\
& \leq C\left(\|\varphi\|_{H^{2}}\right) d_{F}^{2}\left(\mu_{t}, \mu_{t_{0}}\right) .
\end{aligned}
$$

So the result follows from $1-$ and $2-$.

Now we state an additional result about the regularity of the pair of processes $(U, V)$.

Proposition 6.31. The pair of processes $(U, V)$ belongs to $L_{l o c}^{2}\left(\mathbb{R}_{+},\left(H^{1-s}\right)^{2}\right)$.

Proof. Let us set

$$
\begin{aligned}
& U_{t}^{s}=\left(I_{d}-\gamma \triangle\right)^{\frac{-s}{2}} U_{t}, \quad V_{t}^{s}=\left(I_{d}-\gamma \triangle\right)^{\frac{-s}{2}} V_{t}, \quad Z_{t}^{s}=\left(I_{d}-\gamma \triangle\right)^{\frac{-s}{2}} Z_{t}, \\
& W_{t}^{1, s}=\left(I_{d}-\gamma \triangle\right)^{\frac{-s}{2}} W_{t}^{1}, \quad W_{t}^{2, s}=\left(I_{d}-\gamma \triangle\right)^{\frac{-s}{2}} W_{t}^{2} .
\end{aligned}
$$

Given that $U_{t} \in H^{-s}, U_{t}^{s} \in L^{2}\left(\mathbb{T}^{2}\right)$. The same conclusion remains true for $V_{t}^{s}, Z_{t}^{s}, W_{t}^{1, s}$ and $W_{t}^{2, s}$. Now by noticing that $\triangle\left(I_{d}-\gamma \triangle\right)^{\frac{-s}{2}}=\left(I_{d}-\gamma \triangle\right)^{\frac{-s}{2}} \triangle$, we deduce from the evolution equations (6.10) and (6.11) of $(U, V)$ given in Theorem 6.14 that

$$
\begin{aligned}
& U_{t}^{s}=U_{0}^{s}+\gamma \int_{0}^{t} \triangle U_{r}^{s} d r+\beta \int_{0}^{t}\left(I_{d}-\triangle\right)^{\frac{-s}{2}}\left(G_{t}^{S, I}\right)^{*}\left(I_{d}-\gamma \triangle\right)^{\frac{s}{2}} Z_{r}^{s} d r \\
&-\beta \int_{0}^{t}\left(I_{d}-\gamma \triangle\right)^{\frac{-s}{2}}\left(G_{r}^{I}\right)^{*}\left(I_{d}-\gamma \triangle\right)^{\frac{s}{2}} U_{r}^{s} d r-\beta \int_{0}^{t}\left(I_{d}-\gamma \triangle\right)^{\frac{-s}{2}}\left(G_{r}^{S}\right)^{*}\left(I_{d}-\gamma \triangle\right)^{\frac{s}{2}} V_{r}^{s} d r+W_{t}^{1, s}, \\
& V_{t}^{s}=V_{0}^{s}+\gamma \int_{0}^{t} \triangle V_{r}^{s} d r-\beta \int_{0}^{t}\left(I_{d}-\gamma \triangle\right)^{\frac{-s}{2}}\left(G_{r}^{S, I}\right)^{*}\left(I_{d}-\gamma \triangle\right)^{\frac{s}{2}} Z_{r}^{s} d r \\
& \quad+\beta \int_{0}^{t}\left(I_{d}-\gamma \triangle\right)^{\frac{-s}{2}}\left(G_{r}^{I}\right)^{*}\left(I_{d}-\gamma \triangle\right)^{\frac{s}{2}} U_{r}^{s} d r+\beta \int_{0}^{t}\left(I_{d}-\gamma \triangle\right)^{\frac{-s}{2}}\left(G_{r}^{S}\right)^{*}\left(I_{d}-\gamma \triangle\right)^{\frac{s}{2}} V_{r}^{s} d r \\
& \quad-\alpha \int_{0}^{t} V_{r}^{s} d r+W_{t}^{2, s} .
\end{aligned}
$$

Moreover the above system can be rewritten as one equation as follows

where

$$
\left(d U_{t}^{s}, d V_{t}^{s}\right)^{\prime}+A\left(t,\left(U_{t}^{s}, V_{t}^{s}\right)\right) d t=J(t) d t+\left(d W_{t}^{1, s}, d W_{t}^{2, s}\right)^{\prime},
$$

$A\left(t,\left(U_{t}^{s}, V_{t}^{s}\right)\right)=$

$$
\begin{aligned}
& \left(\begin{array}{cc}
-\gamma \triangle+\beta\left(I_{d}-\gamma \triangle\right)^{\frac{-s}{2}}\left(G_{t}^{I}\right)^{*}\left(I_{d}-\gamma \triangle\right)^{\frac{s}{2}} & \beta\left(I_{d}-\gamma \triangle\right)^{\frac{-s}{2}}\left(G_{t}^{S}\right)^{*}\left(I_{d}-\gamma \triangle\right)^{\frac{s}{2}} \\
-\beta\left(I_{d}-\gamma \triangle\right)^{\frac{-s}{2}}\left(G_{t}^{I}\right)^{*}\left(I_{d}-\gamma \triangle\right)^{\frac{s}{2}} & -\gamma \triangle-\beta\left(I_{d}-\gamma \triangle\right)^{\frac{-s}{2}}\left(G_{t}^{S}\right)^{*}\left(I_{d}-\gamma \triangle\right)^{\frac{s}{2}}+\alpha
\end{array}\right)\left(\begin{array}{c}
U_{t}^{s} \\
V_{t}^{s}
\end{array}\right) \\
& J(t)=\left(\left(I_{d}-\gamma \triangle\right)^{\frac{-s}{2}}\left(G_{t}^{S, I}\right)^{*}\left(I_{d}-\gamma \triangle\right)^{\frac{s}{2}} Z_{t}^{s},\right. \\
& \text { and }(u, v)^{\prime} \text { denotes the transpose of the pair }(u, v) .
\end{aligned}
$$

On the other hand given that the dual spaces of $\left(L^{2}\left(\mathbb{T}^{2}\right)\right)^{2}$ and $\left(H^{1}\right)^{2}$ are isomorphic to $\left(L^{2}\left(\mathbb{T}^{2}\right)\right)^{\prime} \times\left(L^{2}\left(\mathbb{T}^{2}\right)\right)^{\prime}$ and $H^{-1} \times H^{-1}$ respectively, by identifying $L^{2}\left(\mathbb{T}^{2}\right)$ to its dual space $\left(L^{2}\left(\mathbb{T}^{2}\right)\right)^{\prime}$, we have $\left(H^{1}\right)^{2} \subset\left(L^{2}\left(\mathbb{T}^{2}\right)\right)^{2} \subset\left(H^{-1}\right)^{2}$.

Let us now prove that the family of operators $A(t,$.$) satisfies the assuptions given in Proposition$ 
2.8 in section 2, with $H=\left(L^{2}\left(\mathbb{T}^{2}\right)\right)^{2}, F=\left(H^{1}\right)^{2}$ and $F^{\prime}=\left(H^{-1}\right)^{2}$.

We first Recall that the family of eigenfunctions $\left(f_{n_{1}, n_{2}}^{i, s}\right)_{i, n_{1}, n_{2}}$ (as defined in Proposition 2.2) associate to the family of eigenvalues $\left(\lambda_{n_{1}, n_{2}}\right)_{n_{1}, n_{2}}$ of the operator $-\gamma \triangle$ is an orthonormal basis of $L^{2}\left(\mathbb{T}^{2}\right)$.

Now we start by showing that $\forall(\mathcal{U}, \mathcal{V}) \in H^{1} \times H^{1}, A(t,(\mathcal{U}, \mathcal{V})) \in H^{-1} \times H^{-1}$. We have $A(t,(\mathcal{U}, \mathcal{V}))=$

$$
\left(\begin{array}{c}
-\gamma \triangle \mathcal{U}+\beta\left(I_{d}-\gamma \triangle\right)^{\frac{-s}{2}}\left(G_{t}^{I}\right)^{*}\left(I_{d}-\gamma \triangle\right)^{\frac{s}{2}} \mathcal{U}+\beta\left(I_{d}-\gamma \triangle\right)^{\frac{-s}{2}}\left(G_{t}^{S}\right)^{*}\left(I_{d}-\gamma \triangle\right)^{\frac{s}{2}} \mathcal{V} \\
-\beta\left(I_{d}-\gamma \triangle\right)^{\frac{-s}{2}}\left(G_{t}^{I}\right)^{*}\left(I_{d}-\gamma \triangle\right)^{\frac{s}{2}} \mathcal{U}-\gamma \triangle \mathcal{V}-\beta\left(I_{d}-\gamma \triangle\right)^{\frac{-s}{2}}\left(G_{t}^{S}\right)^{*}\left(I_{d}-\gamma \triangle\right)^{\frac{s}{2}} \mathcal{V}+\alpha \mathcal{V}
\end{array}\right)
$$

Moreover:

$$
\begin{aligned}
(i) .\left\|\left(I_{d}-\gamma \triangle\right)^{\frac{-s}{2}}\left(G_{t}^{I}\right)^{*}\left(I_{d}-\gamma \triangle\right)^{\frac{s}{2}} \mathcal{U}\right\|_{H^{-1}} & \leq C\left\|\left(I_{d}-\gamma \triangle\right)^{\frac{-s}{2}}\left(G_{t}^{I}\right)^{*}\left(I_{d}-\gamma \triangle\right)^{\frac{s}{2}} \mathcal{U}\right\|_{L^{2}} \\
& =C\left\|\left(G_{t}^{I}\right)^{*}\left(I_{d}-\gamma \triangle\right)^{\frac{s}{2}} \mathcal{U}\right\|_{H^{-s}} \\
& \leq C\left\|\left(I_{d}-\gamma \triangle\right)^{\frac{s}{2}} \mathcal{U}\right\|_{H^{-s}} \\
& =C\|\mathcal{U}\|_{L^{2}}
\end{aligned}
$$

where the second inequality follows from Corollary 6.20.

(ii). Similarly form Corollary 6.20, we have

$$
\begin{aligned}
\left\|\beta\left(I_{d}-\gamma \triangle\right)^{\frac{-s}{2}}\left(G_{t}^{S}\right)^{*}\left(I_{d}-\gamma \triangle\right)^{\frac{s}{2}} \mathcal{V}\right\|_{H^{-1}} & \leq C\left\|\left(I_{d}-\gamma \triangle\right)^{\frac{-s}{2}}\left(G_{t}^{S}\right)^{*}\left(I_{d}-\gamma \triangle\right)^{\frac{s}{2}} \mathcal{V}\right\|_{L^{2}} \\
& =C\left\|\left(G_{t}^{S}\right)^{*}\left(I_{d}-\gamma \triangle\right)^{\frac{s}{2}} \mathcal{V}\right\|_{H^{-s}} \\
& \leq C\left\|\left(I_{d}-\gamma \triangle\right)^{\frac{s}{2}} \mathcal{V}\right\|_{H^{-s}} \\
& =C\|\mathcal{V}\|_{L^{2}} .
\end{aligned}
$$

$$
\begin{aligned}
(\text { iii }) .\|-\gamma \triangle \mathcal{U}\|_{H^{-1}}^{2} & =\sum_{i, n_{1}, n_{2}}\left(1+\gamma \pi^{2}\left(n_{1}^{2}+n_{2}^{2}\right)\right)^{-1}\left(-\gamma \triangle \mathcal{U}, f_{n_{1}, n_{2}}^{i}\right)_{L^{2}}^{2} \\
& =\sum_{i \neq 0, n_{1}, n_{2}} \gamma \pi^{2}\left(n_{1}^{2}+n_{2}^{2}\right)\left(1+\gamma \pi^{2}\left(n_{1}^{2}+n_{2}^{2}\right)\right)^{-1}\left(\mathcal{U}, f_{n_{1}, n_{2}}^{i}\right)_{L^{2}}^{2} \\
& \leq \sum_{i, n_{1}, n_{2}}\left(1+\gamma \pi^{2}\left(n_{1}^{2}+n_{2}^{2}\right)\right)\left(\mathcal{U}, f_{n_{1}, n_{2}}^{i}\right)_{L^{2}}^{2}=\|\mathcal{U}\|_{H^{1}}^{2} .
\end{aligned}
$$

So from $(i),(i i),($ iii $)$ we conclude that $A(t,(\mathcal{U}, \mathcal{V})) \in H^{-1} \times H^{-1}$.

Let us now prove that the four assuptions in Proposition 2.8 are satisfied. In the rest of this proof we will note by $<.$, . $>$ the duality product between $H^{-1} \times H^{-1}$ and $H^{1} \times H^{1}$.

(1). Given that $A(t,$.$) is linear, to prove the first point, it is enough to show that \forall(\mathcal{U}, \mathcal{V})$, $\left(\mathcal{U}_{1}, \mathcal{V}_{1}\right) \in H^{1} \times H^{1}$ the map $\theta \mapsto<A(t, \theta(\mathcal{U}, \mathcal{V})),\left(\mathcal{U}_{1}, \mathcal{V}_{1}\right)>$ is continuous on $\mathbb{R}$, which in turn is so easy.

(2). The fact that there exists $\delta>0$ such that $\|A(t,(\mathcal{U}, \mathcal{V}))\|_{H^{-1} \times H^{-1}} \leq \delta\|(\mathcal{U}, \mathcal{V})\|_{H^{1} \times H^{1}}$ follows from $(i),(i i)$, and $(i i i)$.

(3). Let us prove that there exists $\sigma_{1}>0, \sigma_{2} \in \mathbb{R}$, such that

$$
<A(t,(\mathcal{U}, \mathcal{V})),(\mathcal{U}, \mathcal{V})>+\sigma_{2}\|(\mathcal{U}, \mathcal{V})\|_{L^{2} \times L^{2}}^{2} \geq \sigma_{1}\|(\mathcal{U}, \mathcal{V})\|_{H^{1} \times H^{1}}^{2}
$$

We have $<A(t,(\mathcal{U}, \mathcal{V})),(\mathcal{U}, \mathcal{V})>$

$$
\begin{aligned}
& =<-\gamma \triangle \mathcal{U}+\beta\left(I_{d}-\gamma \triangle\right)^{\frac{-s}{2}}\left(G_{t}^{I}\right)^{*}\left(I_{d}-\gamma \triangle\right)^{\frac{s}{2}} \mathcal{U}+\beta\left(I_{d}-\gamma \triangle\right)^{\frac{-s}{2}}\left(G_{t}^{S}\right)^{*}\left(I_{d}-\gamma \triangle\right)^{\frac{s}{2}} \mathcal{V}, \mathcal{U}> \\
& +<-\beta\left(I_{d}-\gamma \triangle\right)^{\frac{-s}{2}}\left(G_{t}^{I}\right)^{*}\left(I_{d}-\gamma \triangle\right)^{\frac{s}{2}} \mathcal{U}-\gamma \triangle \mathcal{V}-\beta\left(I_{d}-\gamma \triangle\right)^{\frac{-s}{2}}\left(G_{t}^{S}\right)^{*}\left(I_{d}-\gamma \triangle\right)^{\frac{s}{2}} \mathcal{V}+\alpha \mathcal{V}, \mathcal{V}>
\end{aligned}
$$

Furthemore:

(3.1). $<-\gamma \triangle \mathcal{U}, \mathcal{U}>=\gamma<\nabla \mathcal{U}, \nabla \mathcal{U}>=\gamma\|\nabla \mathcal{U}\|_{L^{2}}^{2}$.

(3.2). From $(i)$ and $(i i)$, we note that

$$
\beta\left(I_{d}-\gamma \triangle\right)^{\frac{-s}{2}}\left(G_{t}^{I}\right)^{*}\left(I_{d}-\gamma \triangle\right)^{\frac{s}{2}} \mathcal{U}+\beta\left(I_{d}-\gamma \triangle\right)^{\frac{-s}{2}}\left(G_{t}^{S}\right)^{*}\left(I_{d}-\gamma \triangle\right)^{\frac{s}{2}} \mathcal{V} \in L^{2}\left(\mathbb{T}^{2}\right),
$$

thus as $H^{1} \subset L^{2}\left(\mathbb{T}^{2}\right)$, from $(i)$ and $(i i)$ and from Young's inequality we have 
$\left|<\beta\left(I_{d}-\gamma \triangle\right)^{\frac{-s}{2}}\left(G_{t}^{I}\right)^{*}\left(I_{d}-\gamma \triangle\right)^{\frac{s}{2}} \mathcal{U}+\beta\left(I_{d}-\gamma \triangle\right)^{\frac{-s}{2}}\left(G_{t}^{S}\right)^{*}\left(I_{d}-\gamma \triangle\right)^{\frac{s}{2}} \mathcal{V}, \mathcal{U}>\right|$

$\leq\left\|\beta\left(I_{d}-\gamma \triangle\right)^{\frac{-s}{2}}\left(G_{t}^{I}\right)^{*}\left(I_{d}-\gamma \triangle\right)^{\frac{s}{2}} \mathcal{U}\right\|_{L^{2}}\|\mathcal{U}\|_{L^{2}}+\left\|\beta\left(I_{d}-\gamma \triangle\right)^{\frac{-s}{2}}\left(G_{t}^{S}\right)^{*}\left(I_{d}-\gamma \triangle\right)^{\frac{s}{2}} \mathcal{V}\right\|_{L^{2}}\|\mathcal{U}\|_{L^{2}}$

$\leq C \beta\left(\|\mathcal{U}\|_{L^{2}}^{2}+\|\mathcal{V}\|_{L^{2}}\|\mathcal{U}\|_{L^{2}}\right) \leq C_{1} \beta\left(\|\mathcal{U}\|_{L^{2}}^{2}+\|\mathcal{V}\|_{L^{2}}^{2}\right)$.

Hence

$<\beta\left(I_{d}-\gamma \triangle\right)^{\frac{-s}{2}}\left(G_{t}^{I}\right)^{*}\left(I_{d}-\gamma \triangle\right)^{\frac{s}{2}} \mathcal{U}+\beta\left(I_{d}-\gamma \triangle\right)^{\frac{-s}{2}}\left(G_{t}^{S}\right)^{*}\left(I_{d}-\gamma \triangle\right)^{\frac{s}{2}} \mathcal{V}, \mathcal{U}>$

$+<-\beta\left(I_{d}-\gamma \triangle\right)^{\frac{-s}{2}}\left(G_{t}^{I}\right)^{*}\left(I_{d}-\gamma \triangle\right)^{\frac{s}{2}} \mathcal{U}-\beta\left(I_{d}-\gamma \triangle\right)^{\frac{-s}{2}}\left(G_{t}^{S}\right)^{*}\left(I_{d}-\gamma \triangle\right)^{\frac{s}{2}} \mathcal{V}, \mathcal{V}>$ belongs in the interval $\left[-C_{2} \beta\left(\|\mathcal{U}\|_{L^{2}}^{2}+\|\mathcal{V}\|_{L^{2}}^{2}\right), C_{2} \beta\left(\|\mathcal{U}\|_{L^{2}}^{2}+\|\mathcal{V}\|_{L^{2}}^{2}\right)\right]$.

$(3.3) .<\mathcal{V}, \mathcal{V}\rangle=\|\mathcal{V}\|_{L^{2}}^{2}$.

So from (3.1), (3.2) and (3.3) we see that it is enough to choose $\sigma_{2}=C_{2} \beta+\gamma$ and $\sigma_{1}=\gamma$.

(4). We finish by showing that for any $(\mathcal{U}, \mathcal{V}) \in H^{1} \times H^{1}$ the map $t \mapsto A(t,(\mathcal{U}, \mathcal{V}))$ is Lebesgue measurable from $] 0, T$ [ to $H^{-1} \times H^{-1}$. However since $H^{-1}$ is a separable Hilbert space then according to the Pettis theorem (see Theorem 1.1 page 2 in [20]) it is enough to prove that $\forall\left(\mathcal{U}_{1}, \mathcal{V}_{1}\right) \in H^{1} \times H^{1}$, the map $t \mapsto<A(t,(\mathcal{U}, \mathcal{V})),\left(\mathcal{U}_{1}, \mathcal{V}_{1}\right)>$ is Lebesgue measurable from $] 0, T[$ to $\mathbb{R}$.

Let us prove that the map $t \mapsto<A(t,(\mathcal{U}, \mathcal{V})),\left(\mathcal{U}_{1}, \mathcal{V}_{1}\right)>$ is continuous from $] 0, T[$ to $\mathbb{R}$.

Let $\left.t, t_{0} \in\right] 0, T\left[\right.$, by noticing that $\forall x \in \mathbb{T}^{2}, G_{t}^{I} \varphi(x)=\varphi(x)\left(\mu_{t}^{I}, \frac{K(x, .)}{\left(\mu_{t}, K\right)}\right)=\varphi(x) \mathcal{K}_{t}^{I}(x)$ and $H^{s-1}$ is a Banach algebra, from Lemma 6.29, we have

$$
\begin{aligned}
\mid<\left(I_{d}-\gamma \triangle\right)^{\frac{-s}{2}} & \left(\left(G_{t}^{I}\right)^{*}-\left(G_{t_{0}}^{I}\right)^{*}\right)\left(I_{d}-\gamma \triangle\right)^{\frac{s}{2}} \mathcal{U}, \mathcal{U}_{1}>\mid \\
& =\left|<\left(I_{d}-\gamma \triangle\right)^{\frac{s}{2}} \mathcal{U},\left(G_{t}^{I}-G_{t_{0}}^{I}\right)\left(I_{d}-\gamma \triangle\right)^{\frac{-s}{2}} \mathcal{U}_{1}>\right| \\
& \leq\left\|\left(I_{d}-\gamma \triangle\right)^{\frac{s}{2}} \mathcal{U}\right\|_{H^{1-s}}\left\|\left(G_{t}^{I}-G_{t_{0}}^{I}\right)\left(I_{d}-\gamma \triangle\right)^{\frac{-s}{2}} \mathcal{U}_{1}\right\|_{H^{s-1}} \\
& \leq\left\|\left(I_{d}-\gamma \triangle\right)^{\frac{s}{2}} \mathcal{U}\right\|_{H^{1-s}}\left\|\left(I_{d}-\gamma \triangle\right)^{\frac{-s}{2}} \mathcal{U}_{1}\left(\mathcal{K}_{t}^{I}(.)-\mathcal{K}_{t_{0}}^{I}(.)\right)\right\|_{H^{s-1}} \\
& \leq C\|\mathcal{U}\|_{H^{1}}\left\|\left(I_{d}-\gamma \triangle\right)^{\frac{-s}{2}} \mathcal{U}_{1}\right\|_{H^{s-1}}\left\|\mathcal{K}_{t}^{I}(.)-\mathcal{K}_{t_{0}}^{I}(.)\right\|_{H^{s-1}} \\
& \leq C\|\mathcal{U}\|_{H^{1}}\left\|\mathcal{U}_{1}\right\|_{H^{-1}}\left\|\mathcal{K}_{t}^{I}(.)-\mathcal{K}_{t_{0}}^{I}(.)\right\|_{H^{2}} \\
& \leq C\|\mathcal{U}\|_{H^{1}}\left\|\mathcal{U}_{1}\right\|_{H^{1}}\left(d_{F}\left(\mu_{t}^{I}, \mu_{t_{0}}^{I}\right)+d_{F}\left(\mu_{t}^{I}, \mu_{t_{0}}^{I}\right)\right) .
\end{aligned}
$$

Similarly by noticing that $\forall \mathcal{U}_{1} \in H^{1},\left(I_{d}-\gamma \triangle\right)^{\frac{-s}{2}} \mathcal{U}_{1} \in H^{1+s} \subset H^{2}$ and

$$
\begin{aligned}
& G_{t}^{S}\left(I_{d}-\gamma \triangle\right)^{\frac{-s}{2}} \mathcal{U}_{1}(x)=\frac{\left(\mu_{t}^{S},\left(I_{d}-\gamma \triangle\right)^{\frac{-s}{2}} \mathcal{U}_{1} K(x, .)\right)}{\left(\mu_{t}, K\right)}, \text { from Lemma 6.30, we have } \\
& \begin{aligned}
\mid<\left(I_{d}-\gamma \triangle\right)^{\frac{-s}{2}} & \left(\left(G_{t}^{S}\right)^{*}-\left(G_{t_{0}}^{S}\right)^{*}\right)\left(I_{d}-\gamma \triangle\right)^{\frac{s}{2}} \mathcal{V}, \mathcal{U}_{1}>\mid \\
& =\left|<\left(I_{d}-\gamma \triangle\right)^{\frac{s}{2}} \mathcal{V},\left(G_{t}^{S}-G_{t_{0}}^{S}\right)\left(I_{d}-\gamma \triangle\right)^{\frac{-s}{2}} \mathcal{U}_{1}>\right| \\
& \leq\left\|\left(I_{d}-\gamma \triangle\right)^{\frac{s}{2}} \mathcal{V}\right\|_{H^{1-s}}\left\|\left(G_{t}^{S}-G_{t_{0}}^{S}\right)\left(I_{d}-\gamma \triangle\right)^{\frac{-s}{2}} \mathcal{U}_{1}\right\|_{H^{s-1}} \\
& \leq\|\mathcal{V}\|_{H^{1}}\|\| \mathcal{K}_{t}^{S}\left(.,\left(I_{d}-\gamma \triangle\right)^{\frac{-s}{2}} \mathcal{U}_{1}\right)-\mathcal{K}_{t_{0}}^{S}\left(.,\left(I_{d}-\gamma \triangle\right)^{\frac{-s}{2}} \mathcal{U}_{1}\right) \|_{H^{2}} \\
& \leq C\left(\|\mathcal{V}\|_{H^{1}},\left\|\mathcal{U}_{1}\right\|_{H^{1}}\right)\left(d_{F}\left(\mu_{t}^{S}, \mu_{t_{0}}^{S}\right)+d_{F}\left(\mu_{t}, \mu_{t_{0}}\right)\right) .
\end{aligned}
\end{aligned}
$$

So

$$
\begin{aligned}
& \mid<A(t,(\mathcal{U}, \mathcal{V})),\left(\mathcal{U}_{1}, \mathcal{V}_{1}\right)>-<A\left(t_{t_{0}},(\mathcal{U}, \mathcal{V})\right),\left(\mathcal{U}_{1}, \mathcal{V}_{1}\right)>\mid \\
& \leq C\left(\|\mathcal{V}\|_{H^{1}},\left\|\mathcal{U}_{1}\right\|_{H^{1}},\|\mathcal{U}\|_{H^{1}},\left\|\mathcal{V}_{1}\right\|_{H^{1}}\right)\left(d_{F}\left(\mu_{t}^{S}, \mu_{t_{0}}^{S}\right)+d_{F}\left(\mu_{t}^{I}, \mu_{t_{0}}^{I}\right)+d_{F}\left(\mu_{t}, \mu_{t_{0}}\right)\right)
\end{aligned}
$$

thus the result follows from the fact that $\mu^{S}, \mu^{I}$ and $\mu$ belongs to $C\left(\mathbb{R}_{+},\left(\mathcal{M}_{F}\left(\mathbb{T}^{2}\right), d_{F}\right)\right)$.

Now in addition to the fact that the family of operators $A(t,$.$) satisfies the four assumptions$ given in Proposition 2.8 in section 2, with $H=\left(L^{2}\left(\mathbb{T}^{2}\right)\right)^{2}, F=\left(H^{1}\right)^{2}$ and $F^{\prime}=\left(H^{-1}\right)^{2}$, we note that:

$-J(t)=\left(\left(I_{d}-\gamma \triangle\right)^{\frac{-s}{2}}\left(G_{t}^{S, I}\right)^{*}\left(I_{d}-\gamma \triangle\right)^{\frac{s}{2}} Z_{t}^{s},-\left(I_{d}-\gamma \triangle\right)^{\frac{-s}{2}}\left(G_{t}^{S, I}\right)^{*}\left(I_{d}-\gamma \triangle\right)^{\frac{s}{2}} Z_{t}^{s}\right)^{\prime}$

belongs to $L_{\text {loc }}^{2}\left(\mathbb{R}_{+},\left(L^{2}\left(\mathbb{T}^{2}\right)\right)^{2}\right)$. Indeed, 


$$
\begin{aligned}
& \left\|\left(I_{d}-\gamma \triangle\right)^{\frac{-s}{2}}\left(G_{r}^{S, I}\right)^{*}\left(I_{d}-\gamma \triangle\right)^{\frac{s}{2}} Z_{r}^{s}\right\|_{L^{2}}=\left\|\left(G_{t}^{S, I}\right)^{*} Z_{t}\right\|_{H^{-s}} \leq C\left\|Z_{t}\right\|_{H^{-s}} \text { and } Z \in C\left(\mathbb{R}_{+}, H^{-s}\right) . \\
- & \left(U_{0}^{s}, V_{0}^{s}\right) \in\left(L^{2}\left(\mathbb{T}^{2}\right)\right)^{2} .
\end{aligned}
$$

Thus we deduce from Proposition 2.8 that for $(A(t, .))_{\{t \in] 0, T[\}}$ being the above family of operators, the stochastic differential equation

$$
\begin{gathered}
(d \mathcal{U}(t), d \mathcal{V}(t))^{\prime}+A\left(t,(\mathcal{U}(t), \mathcal{V}(t)) d t=J(t) d t+\left(d W_{t}^{1, s}, d W_{t}^{2, s}\right)^{\prime}\right. \\
(\mathcal{U}(0), \mathcal{V}(0))=\left(U_{0}^{s}, V_{0}^{s}\right)
\end{gathered}
$$

admits a unique solution $(\mathcal{U}, \mathcal{V}) \in L_{\text {loc }}^{2}\left(\mathbb{R}_{+},\left(H^{1}\left(\mathbb{T}^{2}\right)\right)^{2}\right) \cap C\left(\mathbb{R}_{+},\left(L^{2}\left(\mathbb{T}^{2}\right)\right)^{2}\right)$ a.s.

On the other hand, it has been shwon in Proposition 6.28, that the pair $\left(U^{s}, V^{s}\right)$ is the unique solution of the equation (6.28) in $C\left(\mathbb{R}_{+},\left(L^{2}\left(\mathbb{T}^{2}\right)\right)^{2}\right)$ (since equation (6.28) is equivalent to the system formed by equations (6.10) and (6.11), which in turn is the same as the system formed by equations (6.26) and (6.27)). Thus we conclude that the pair of processes $\left(U^{s}, V^{s}\right)=(\mathcal{U}, \mathcal{V})$ which belongs to $L_{\text {loc }}^{2}\left(\mathbb{R}_{+},\left(H^{1}\left(\mathbb{T}^{2}\right)\right)^{2}\right) \cap C\left(\mathbb{R}_{+},\left(L^{2}\left(\mathbb{T}^{2}\right)\right)^{2}\right)$, consequently the pair of processes $(U, V)$ belongs to $L_{l o c}^{2}\left(\mathbb{R}_{+},\left(H^{1-s}\right)^{2}\right)$.

Remark 6.32. Given that $d Z_{t}^{N}=\gamma \triangle Z_{t}^{N} d t+d \widetilde{\mathcal{H}}_{t}^{N}$ (see equation (6.1)), by proceeding as in the proof of Proposition 6.31, it is easy to see that $\forall N \in \mathbb{N}^{*}, Z^{N} \in L_{\text {loc }}^{2}\left(\mathbb{R}_{+}, H^{1-s}\right)$. Furthemore by Using the Itô formula establish in [20 page 62] we can see that the sequence $\left(Z^{N}\right)_{N}$ is bounded in $L_{\text {loc }}^{2}\left(\mathbb{R}_{+}, H^{1-s}\right)$ (although we do not establish this result in this paper). Consequently since in a Hilbert space the bounded sets are relatively compact for the weak topology, the convergence in law of $\left(Z^{N}\right)_{N}$ in $L_{l o c}^{2}\left(\mathbb{R}_{+}, H^{1-s}\right)$ equipped with its weak topology can be easily deduced.

The convergence in law of the sequence $\left(U^{N}, V^{N}\right)_{N}$ in $\left(L_{l o c}^{2}\left(\mathbb{R}_{+}, H^{1-s}\right)\right)^{2}$ where

$L_{\text {loc }}^{2}\left(\mathbb{R}_{+}, H^{1-s}\right)$ is equipped with its weak topology follows also by the fact it is bounded in $\left(L_{\text {loc }}^{2}\left(\mathbb{R}_{+}, H^{1-s}\right)\right)^{2}$, which in turn is not difficult to prove by using the Proposition 6.31. However we do not establish that result in this paper.

\section{Law of Large Numbers, Central Limit Theorem of the sequence $\left(\mu^{S, N}, \mu^{I, N}\right)_{N \geq 1}$ : the case $\gamma=0$}

In this section we consider the second model presented in the Introduction ie when the diffusion coefficient $\gamma=0$. More precisely we consider a compartmental SIR stochastic epidemic model for a population distributed on the two dimentional torus such that:

- The position of an individual $i$ is independent of time and is represented by $X^{i}$ defined as in the Introduction.

- A susceptible $i$ become infected at time t at the rate $\beta 1_{\left\{E_{t}^{i}=S\right\}} \sum_{j=1}^{N} \frac{K\left(X^{i}, X^{j}\right)}{\sum_{l=1}^{N} K\left(X^{l}, X^{j}\right)} 1_{\left\{E_{t}^{j}=I\right\}}$,

where $\alpha, \beta, E_{t}^{i}$ and the function $\mathrm{K}$ are defined as in the Introduction.

The temporal evolution of $S(\cdot), I(\cdot)$ and $R(\cdot)$ are defined as in the Introduction.

The assumptions made at time $t=0$, are presented in the Introduction, in other words, the sequence of empirical measures $\left(\mu_{0}^{S, N}, \mu_{0}^{I, N}, \mu_{0}^{N}\right)_{N}$ is defined as in the Introduction.

Thus the renormalized processes $\mu^{S, N}, \mu^{I, N}, \mu^{R, N}$ and $\mu^{N}$ are defined as follows. $\forall t>0$,

$$
\begin{aligned}
& \mu_{t}^{S, N}=\frac{1}{N} \sum_{i=1}^{N} 1_{\left\{E_{t}^{i}=S\right\}} \delta_{X^{i}} \\
& \mu_{t}^{I, N}=\frac{1}{N} \sum_{i=1}^{N} 1_{\left\{E_{t}^{i}=I\right\}} \delta_{X^{i}} \\
& \mu_{t}^{R, N}=\frac{1}{N} \sum_{i=1}^{N} 1_{\left\{E_{t}^{i}=R\right\}} \delta_{X^{i}} \\
& \mu^{N}=\mu_{t}^{S, N}+\mu_{t}^{I, N}+\mu_{t}^{R, N}=\frac{1}{N} \sum_{i=1}^{N} \delta_{X^{i}} .
\end{aligned}
$$


We first recall that Assumption (H0) in section 3 remains true. Now since we have already shown in section 3 and section 4 respectively that $\left(\mu_{0}^{S, N}, \mu_{0}^{I, N}, \mu^{N}\right) \stackrel{a . s}{\rightarrow}\left(\mu_{0}^{S}, \mu_{0}^{I}, \mu\right)$ and that $\left(U_{0}^{N}=\sqrt{N}\left(\mu_{0}^{S, N}-\mu_{0}^{S}\right), V_{0}^{N}=\sqrt{N}\left(\mu_{0}^{I, N}-\mu_{0}^{I}\right), Z^{N}=\sqrt{N}\left(\mu^{N}-\mu\right)\right)$ converges in law in $\left(H^{-s}\right)^{3}$ (for any $\mathrm{s}>1$ ) towards the Gaussian vector $\left(U_{0}, V_{0}, Z\right)$, then the aim in this section is to study the law of large numbers and the central limit theorem of the sequence $\left\{\left(\mu_{t}^{S, N}, \mu_{t}^{I, N}\right), t \geq 0, N \geq 1\right\}$.

\subsection{Law of Large Numbers}

The following is assumed to hold throughout subsection 7.1.

\section{Assumption (H3): $\mathrm{k}$ is Lipschitz.}

\subsubsection{System of evolution equations of the pair $\left(\mu^{S, N}, \mu^{I, N}\right)$}

For $\varphi \in C\left(\mathbb{T}^{2}\right)$, since $\gamma=0$, the evolution equations of $\mu^{S, N}$ and $\mu^{I, N}$ simplify as follows.

$\left(\mu_{t}^{S, N}, \varphi\right)=\left(\mu_{0}^{S, N}, \varphi\right)-\beta \int_{0}^{t}\left(\mu_{r}^{S, N}, \varphi\left(\mu_{r}^{I, N}, \frac{K}{\left(\mu^{N}, K\right)}\right)\right) d r+M_{t}^{\prime N, \varphi}$

$\left(\mu_{t}^{I, N}, \varphi\right)=\left(\mu_{0}^{I, N}, \varphi\right)+\int_{0}^{t}\left(\mu_{r}^{S, N}, \varphi\left(\mu_{r}^{I, N}, \frac{K}{\left(\mu^{N}, K\right)}\right)\right) d r-\alpha \int_{0}^{t}\left(\mu_{r}^{I, N}, \varphi\right) d r+L_{t}^{\prime N, \varphi}$,

where

$$
\begin{aligned}
M_{t}^{\prime N, \varphi} & =-\frac{1}{N} \sum_{i=1}^{N} \int_{0}^{t} \int_{0}^{\infty} 1_{\left\{E_{r^{-}}^{i}=S\right\}} \varphi\left(X^{i}\right) 1_{\left\{u \leq \frac{\beta}{N} \sum_{j=1}^{N} \frac{K\left(X^{i}, X^{j}\right)}{\sum_{l=1}^{N} K\left(X^{l}, X^{j}\right)} 1_{\left\{E_{r}^{j}=I\right\}}\right.} \bar{M}^{i}(d r, d u), \\
L_{t}^{\prime N, \varphi} & \left.=\frac{1}{N} \sum_{i=1}^{N} \int_{0}^{t} \int_{0}^{\infty} 1_{\left\{E_{r^{-}}^{i}=S\right\}} \varphi\left(X^{i}\right) 1_{\left\{u \leq \frac{\beta}{N} \sum_{j=1}^{N} \frac{K\left(X^{i}, X^{j}\right)}{\sum_{l=1}^{N} K\left(X^{l}, X^{j}\right)} 1_{\left\{E_{r}^{j}=I\right\}}\right\}} \bar{M}^{i}(d r, d u)\right) \\
& -\frac{1}{N} \sum_{i=1}^{N} \int_{0}^{t} \int_{0}^{\alpha} 1_{\left\{E_{r^{-}}^{i}=I\right\}} \varphi\left(X^{i}\right) \bar{Q}^{i}(d r, d u) .
\end{aligned}
$$

Let us state the main result of this subsection.

Theorem 7.1. The sequence $\left(\mu^{S, N}, \mu^{I, N}\right)_{N \geq 1}$ converges in probability in $\left(D\left(\mathbb{R}_{+}, \mathcal{M}\left(\mathbb{T}^{2}\right)\right)\right)^{2}$ towards $\left(\mu^{S}, \mu^{I}\right) \in\left(C\left(\mathbb{R}_{+}, \mathcal{M}\left(\mathbb{T}^{2}\right)\right)\right)^{2}$ where $\left\{\left(\mu_{t}^{S}, \mu_{t}^{I}\right), t \geq 0\right\}$ satisfies, $\forall \varphi \in C\left(\mathbb{T}^{2}\right)$,

$$
\begin{aligned}
& \left(\mu_{t}^{S}, \varphi\right)=\left(\mu_{0}^{S}, \varphi\right)-\beta \int_{0}^{t}\left(\mu_{r}^{S}, \varphi\left(\mu_{r}^{I}, \frac{K}{(\mu, K)}\right)\right) d r \\
& \left(\mu_{t}^{I}, \varphi\right)=\left(\mu_{0}^{I}, \varphi\right)+\beta \int_{0}^{t}\left(\mu_{r}^{S}, \varphi\left(\mu_{r}^{I}, \frac{K}{(\mu, K)}\right)\right) d r-\alpha \int_{0}^{t}\left(\mu_{r}^{I}, \varphi\right) d r .
\end{aligned}
$$

Proof. We obtain the tightness of the sequence $\left(\mu^{S, N}, \mu^{I, N}\right)_{N \geq 1}$ by an adaptation of the proof of Proposition 5.4, thus by Prokhorov's theorem we deduce the existence of a subsequence which converges in law towards $\left\{\left(\mu_{t}^{S}, \mu_{t}^{I}\right), t \geq 0\right\}$. Furthemore, adapting the proof of Theorem 5.6 we prove that $\left\{\left(\mu_{t}^{S}, \mu_{t}^{I}\right), t \geq 0\right\}$ is continuous and verifies the system formed by the equations (7.1) and (7.2), and $\forall t \geq 0, \mu_{t}^{S}$ and $\mu_{t}^{I}$ are absolutely continuous with respect to the Lebesgue measue with densities $f_{S}(t,$.$) and f_{S}(t,$.$) bounded by \delta_{2}\left(\delta_{2}\right.$ is defined in section 3$)$.

On the other hand $\left(f_{S}(t,),. f_{I}(t,).\right)$ satisfies the following system.

$$
\begin{aligned}
& f_{S}(t)=f_{S}(0)-\beta \int_{0}^{t} f_{S}(r) \int_{\mathbb{T}_{2}} \frac{K(., y)}{\int_{\mathbb{T}^{2}} K\left(x^{\prime}, y\right) g\left(x^{\prime}\right) d x^{\prime}} f_{I}(r, y) d y d r \\
& f_{I}(t)=f_{I}(0)+\beta \int_{0}^{t} f_{S}(r) \int_{\mathbb{T}_{2}} \frac{K(., y)}{\int_{\mathbb{T}^{2}} K\left(x^{\prime}, y\right) g\left(x^{\prime}\right) d x^{\prime}} f_{I}(r, y) d y d r-\alpha \int_{0}^{t} f_{I}(r) d r,
\end{aligned}
$$


where $g$ is the density of $\mu$. Moreover it is easy to prove that the above system admits a unique solution in the set $\Lambda=\left\{\left(f_{1}, f_{2}\right) / 0 \leq f_{i} \leq \delta_{2}, i \in\{1,2\}\right\}$. Thus we conclude that the sequence $\left(\mu^{S, N}, \mu^{I, N}\right)_{N \geq 1}$ weakly converges in $\left(D\left(\mathbb{R}_{+}, \mathcal{M}\left(\mathbb{T}^{2}\right)\right)\right)^{2}$ towards $\left(\mu^{S}, \mu^{I}\right)$. However as the initial measures $\mu_{0}^{S}$ and $\mu_{0}^{I}$ are deterministic (see Theorem 3.1 in section 3 ), the measures $\mu_{t}^{S}$ and $\mu_{t}^{I}$ also have this property, consequently we have the convergence in probability.

\subsection{Central Limit Theorem}

The aim here is to study the convergence of the sequence $\left(U^{N}=\sqrt{N}\left(\mu^{S, N}-\mu^{S}\right), V^{N}=\right.$ $\left.\sqrt{N}\left(\mu^{I, N}-\mu^{I}\right)\right)$, as $N \rightarrow \infty$ in $D\left(\mathbb{R}_{+}, H^{-s}\right) \times D\left(\mathbb{R}_{+}, H^{-s}\right)$ with s $>1$ (the choice of such a 's' is justified as in subsection 6.2). To this end we make the following assumptions.

Assuptions (H4): $k \in C^{2}\left(\mathbb{R}_{+}\right)$.

Following the same argument as that used in the proof of lemma 6.2, we note that:

Remark 7.2. Under the assumption $(H 4), \sup _{x}\|K(x, .)\|_{H^{s}}<\infty$

\subsubsection{Equations verified by the pair $\left(U^{N}, V^{N}\right)$}

Let $\varphi \in C\left(\mathbb{T}^{2}\right)$, by a similar reasoning as in subsection 6.1 , we see that

$$
\begin{aligned}
\left(U_{t}^{N}, \varphi\right)= & \left(U_{0}^{N}, \varphi\right)+\beta \int_{0}^{t}\left(Z^{N}, G_{r}^{S, I, N} \varphi\right) d r-\beta \int_{0}^{t}\left(U_{r}^{N}, G_{r}^{I, N} \varphi\right) d r-\beta \int_{0}^{t}\left(V_{r}^{N}, G_{r}^{S} \varphi\right) d r+\widetilde{M}_{t}^{\prime N, \varphi}, \\
\left(V_{t}^{N}, \varphi\right)= & \left(V_{0}^{N}, \varphi\right)-\beta \int_{0}^{t}\left(Z^{N}, G_{r}^{S, I, N} \varphi\right) d r+\beta \int_{0}^{t}\left(U_{r}^{N}, G_{r}^{I, N} \varphi\right) d r+\beta \int_{0}^{t}\left(V_{r}^{N}, G_{r}^{S} \varphi\right) d r \\
& -\alpha \int_{0}^{t}\left(V_{r}^{N}, \varphi\right) d r+\widetilde{L}_{t}^{\prime N, \varphi}
\end{aligned}
$$

where

$$
\begin{aligned}
& -\widetilde{M}_{t}^{\prime N, \varphi}=\sqrt{N} M_{t}^{\prime N, \varphi} \text { and } \widetilde{L}_{t}^{\prime N, \varphi}=\sqrt{N} L_{t}^{\prime N, \varphi} \\
& -\forall x, y, x^{\prime} \in \mathbb{T}_{2}, \\
& G_{r}^{S, I, N} \varphi\left(x^{\prime}\right)=\left(\mu_{r}^{I, N}, K\left(x^{\prime}, .\right) \frac{\left(\mu_{r}^{S, N}, \varphi K\right)}{\left(\mu^{N}, K\right)(\mu, K)}\right) \\
& \quad=\int_{\mathbb{T}^{2}} K\left(x^{\prime}, y\right) \frac{\int_{\mathbb{T}^{2}} \varphi(x) K(x, y) \mu_{r}^{S, N}(d x)}{\int_{\mathbb{T}^{2}} K\left(y^{\prime}, y\right) \mu^{N}\left(d y^{\prime}\right) \int_{\mathbb{T}^{2}} K\left(y^{\prime}, y\right) \mu\left(d y^{\prime}\right)} \mu_{r}^{I, N}(d y), \\
& G_{r}^{I, N} \varphi(x)=\varphi(x)\left(\mu_{r}^{I, N}, \frac{K(x, .)}{(\mu, K)}\right)=\varphi(x) \int_{\mathbb{T}^{2}} \frac{K(x, y)}{\int_{\mathbb{T}^{2}} K\left(y^{\prime}, y\right) \mu\left(d y^{\prime}\right)} \mu_{r}^{I, N}(d y), \\
& G_{r}^{S} \varphi(y)=\frac{\left(\mu_{r}^{S}, \varphi K(., y)\right)}{(\mu, K(., y))}=\frac{\int_{\mathbb{T}^{2}} \varphi(x) K(x, y) \mu_{r}^{S}(d x)}{\int_{\mathbb{T}^{2}} K\left(y^{\prime}, y\right) \mu\left(d y^{\prime}\right)} .
\end{aligned}
$$

In the rest of this section we arbitrarily choose $1<s<2$, and we equipped $D\left(\mathbb{R}_{+}, H^{-s}\right)$ with the Skorhokod topology. 
Theorem 7.3. Under $\left(H_{4}\right)$, the sequence $\left(U^{N}, V^{N}\right)_{N \geq 1}$ converges in law in $\left(D\left(\mathbb{R}_{+}, H^{-s}\right)\right)^{2}$ to the pair of process $(U, V)$ which belongs to $\left(C\left(\mathbb{R}_{+}, H^{-s}\right)\right)^{2}$ and satisfies:

$$
\begin{aligned}
& U_{t}=U_{0}+\beta \int_{0}^{t}\left(G_{r}^{S, I}\right)^{*} Z d r-\beta \int_{0}^{t}\left(G_{r}^{I}\right)^{*} U_{r} d r-\beta \int_{0}^{t}\left(G_{r}^{S}\right)^{*} V_{r} d r+W_{t}^{\prime 1}, \\
& V_{t}=V_{0}-\beta \int_{0}^{t}\left(G_{r}^{S, I}\right)^{*} Z d r+\beta \int_{0}^{t}\left(G_{r}^{I}\right)^{*} U_{r} d r+\beta \int_{0}^{t}\left(G_{r}^{S}\right)^{*} V_{r} d r-\alpha \int_{0}^{t} V_{r} d r+W_{t}^{\prime 2},
\end{aligned}
$$

where $\forall \varphi, \psi \in H^{s},\left(\left(W^{\prime 1}, \varphi\right),\left(W^{\prime 2}, \psi\right)\right)$ is a centered Gaussian martingale verifying

$$
\begin{aligned}
& <\left(W^{\prime 1}, \varphi\right)>_{t}=\beta \int_{0}^{t}\left(\mu_{r}^{S}, \varphi^{2}\left(\mu_{r}^{I}, \frac{K}{(\mu, K)}\right)\right) d r, \\
& <\left(W^{\prime 2}, \psi\right)>_{t}=\beta \int_{0}^{t}\left(\mu_{r}^{S}, \psi^{2}\left(\mu_{r}^{I}, \frac{K}{(\mu, K)}\right)\right) d r+\alpha \int_{0}^{t}\left(\mu_{r}^{I}, \psi^{2}\right) d r, \\
& <\left(W^{\prime 1}, \varphi\right),\left(W^{\prime 2}, \psi\right)>_{t}=-\beta \int_{0}^{t}\left(\mu_{r}^{S}, \varphi \psi\left(\mu_{r}^{I}, \frac{K}{(\mu, K)}\right)\right) d r .
\end{aligned}
$$

The proof of the next Proposition, which will be useful for the proof of Theorem 7.3 is an easy adaptation of the proof of Proposition 6.18.

Proposition 7.4. The sequence $\left\{\left(\widetilde{M}_{t}^{\prime} N, \widetilde{L}_{t}^{\prime} N\right), t \geq 0\right\}_{N \geq 1}$ converges in law in $\left(D\left(\mathbb{R}_{+}, H^{-s}\right)\right)^{2}$ towards the process $\left(W^{\prime 1}, W^{\prime 2}\right) \in\left(C\left(\mathbb{R}_{+}, H^{-s}\right)\right)^{2}$ and $\forall \varphi, \psi \in H^{s},\left(\left(W^{\prime 1}, \varphi\right),\left(W^{\prime 2}, \psi\right)\right)$ is a centered Gaussian martingale of the form

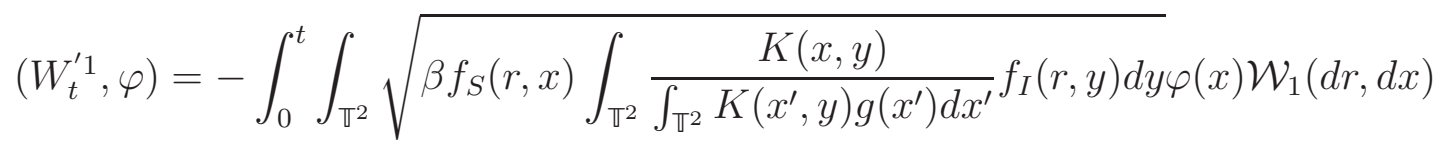

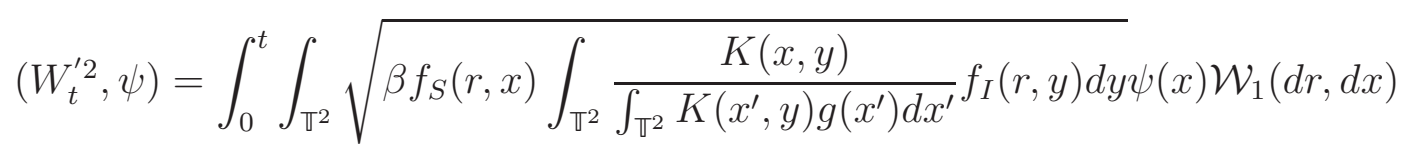

$$
\begin{aligned}
& -\int_{0}^{t} \int_{\mathbb{T}^{2}} \psi(x) \sqrt{\alpha f_{I}(r, x)} \mathcal{W}_{2}(d r, d x),
\end{aligned}
$$

where $g$ is the density of $\mu$ and $\mathcal{W}_{1}, \mathcal{W}_{2}$ are independent spatio-temporal white noises.

\subsubsection{Proof of Theorem 7.3}

We establish the tightness of the sequence $\left(U^{N}, V^{N}\right)$ first, then we show that all converging subsequences of $\left(U^{N}, V^{N}\right)_{N \geq 1}$ have the same limit which we shall identify.

The next proposition is useful to prove the tightness of the sequence $\left(U^{N}, V^{N}\right)_{N \geq 1}$.

Proposition 7.5. $\forall T>0$,

$$
\begin{aligned}
& \sup _{N \geq 1} \mathbb{E}\left(\sup _{0 \leq t \leq T}\left\|U_{t}^{N}\right\|_{H^{-s}}^{2}\right)<\infty, \\
& \sup _{N \geq 1} \mathbb{E}\left(\sup _{0 \leq t \leq T}\left\|V_{t}^{N}\right\|_{H^{-s}}^{2}\right)<\infty .
\end{aligned}
$$

Proof. From equations (7.3) and (7.4), we have

$$
\begin{aligned}
\left\|U_{t}^{N}\right\|_{H^{-s}}^{2} \leq & 5\left\|U_{0}^{N}\right\|_{H^{-s}}^{2}+5 \beta^{2} t \int_{0}^{t}\left\|\left(G_{r}^{S, I, N}\right)^{*} Z^{N}\right\|_{H^{-s}}^{2} d r+5 \beta^{2} t \int_{0}^{t}\left\|\left(G_{r}^{I, N}\right)^{*} U_{r}^{N}\right\|_{H^{-s}}^{2} d r \\
& +5 \beta^{2} t \int_{0}^{t}\left\|\left(G_{r}^{S}\right)^{*} V_{r}^{N}\right\|_{H^{-s}}^{2} d r+5\left\|\widetilde{M}_{t}^{\prime N, \varphi}\right\|_{H^{-s}}^{2}, \\
\left\|V_{t}^{N}\right\|_{H^{-s}}^{2} \leq & 6\left\|V_{0}^{N}\right\|_{H^{-s}}^{2}+6 \beta^{2} t \int_{0}^{t}\left\|\left(G_{r}^{S, I, N}\right)^{*} Z^{N}\right\|_{H^{-s}}^{2} d r+6 \beta^{2} t \int_{0}^{t}\left\|\left(G_{r}^{I, N}\right)^{*} U_{r}^{N}\right\|_{H^{-s}}^{2} d r \\
& +6 \beta^{2} t \int_{0}^{t}\left\|\left(G_{r}^{S}\right)^{*} V_{r}^{N}\right\|_{H^{-s}}^{2} d r+6 \alpha^{2} t \int_{0}^{t}\left\|V_{r}^{N}\right\|_{H^{-s}}^{2} d r+6\left\|\widetilde{L}_{t}^{\prime N, \varphi}\right\|_{H^{-s} .}^{2} .
\end{aligned}
$$


So since Proposition 6.19 and Corollary 6.20 remain true for $\mu_{t}=\mu$, we have

$$
\begin{aligned}
\left\|U_{t}^{N}\right\|_{H^{-s}}^{2} \leq & 5\left\|U_{0}^{N}\right\|_{H^{-s}}^{2}+5 \beta^{2} t^{2} C \sup _{y}\|K(., y)\|_{H^{s}}^{2}\left\|Z^{N}\right\|_{H^{-s}}^{2}+5 \beta^{2} t C \sup _{y}\|K(., y)\|_{H^{s}}^{2} \int_{0}^{t}\left\|U_{r}^{N}\right\|_{H^{-s}}^{2} d r \\
& +5 \beta^{2} t C \sup _{x}\left\|\frac{K(x, .)}{\int_{\mathbb{T}^{2}} K\left(x^{\prime}, .\right) \mu\left(d x^{\prime}\right)}\right\|_{H^{s}}^{2} \int_{0}^{t}\left\|V_{r}^{N}\right\|_{H^{-s}}^{2} d r+5\left\|\widetilde{M}_{t}^{N, \varphi}\right\|_{H^{-s}}^{2}, \\
\left\|V_{t}^{N}\right\|_{H^{-s}}^{2} \leq & 6\left\|V_{0}^{N}\right\|_{H^{-s}}^{2}+6 \beta^{2} t^{2} C \sup _{y}\|K(., y)\|_{H^{s}}^{2}\left\|Z^{N}\right\|_{H^{-s}}^{2}+6 \beta^{2} t C \sup _{y}\|K(., y)\|_{H^{s}}^{2} \int_{0}^{t}\left\|U_{r}^{N}\right\|_{H^{-s}}^{2} d r \\
+ & 6 t\left(C \beta^{2} \sup _{x}\left\|\frac{K(x, .)}{\int_{\mathbb{T}^{2}} K\left(x^{\prime}, .\right) \mu\left(d x^{\prime}\right)}\right\|_{H^{s}}^{2}+\alpha^{2}\right) \int_{0}^{t}\left\|V_{r}^{N}\right\|_{H^{-s}}^{2} d r+6\left\|\widetilde{L}_{t}^{\prime N, \varphi}\right\|_{H^{-s}}^{2} .
\end{aligned}
$$

Thus from Remark 7.2 and Lemma 8.2 below, one has

$$
\begin{aligned}
\mathbb{E}\left(\sup _{0 \leq l \leq t}\left\|U_{l}^{N}\right\|_{H^{-s}}^{2}\right) \leq & 5 \sup _{N \geq 1} \mathbb{E}\left(\left\|U_{0}^{N}\right\|_{H^{-s}}\right)+5 t \beta^{2} C \int_{0}^{t}\left\{\mathbb{E}\left(\sup _{0 \leq u \leq r}\left\|U_{u}^{N}\right\|_{H^{-s}}^{2}\right)+\mathbb{E}\left(\sup _{0 \leq u \leq r}\left\|V_{u}^{N}\right\|_{H^{-s}}^{2}\right)\right\} d r \\
& +5 t^{2} \beta^{2} C \sup _{N \geq 1} \mathbb{E}\left(\left\|Z^{N}\right\|_{H^{-s}}\right)+\sup _{N \geq 1}\left(\sup _{0 \leq r \leq t}\left\|\widetilde{M}_{r}^{\prime N}\right\|_{H^{-s}}\right),
\end{aligned}
$$

$\mathbb{E}\left(\sup _{0 \leq l \leq t}\left\|V_{l}^{N}\right\|_{H^{-s}}^{2}\right) \leq 6 \sup _{N \geq 1} \mathbb{E}\left(\left\|V_{0}^{N}\right\|_{H^{-s}}^{2}\right)$

$$
\begin{aligned}
& +6 t\left(\beta^{2} C+\alpha^{2}\right) \int_{0}^{t}\left\{\mathbb{E}\left(\sup _{0 \leq u \leq r}\left\|U_{u}^{N}\right\|_{H^{-s}}^{2}\right)+\mathbb{E}\left(\sup _{0 \leq u \leq r}\left\|V_{u}^{N}\right\|_{H^{-s}}^{2}\right)\right\} d r \\
& +6 t^{2} \beta^{2} C \sup _{N \geq 1} \mathbb{E}\left(\left\|Z^{N}\right\|_{H^{-s}}^{2}\right)+\sup _{N \geq 1} \mathbb{E}\left(\sup _{0 \leq r \leq t}\left\|\widetilde{L}_{r}^{\prime N}\right\|_{H^{-s}}\right) .
\end{aligned}
$$

Hence summing (7.7) and (7.8) and applying Gronwall's lemma we obtain the result from Corollary 6.4 and Proposition 4.1 in section 4.

Now we prove the tightness of the sequence $\left(U^{N}, V^{N}\right)_{N \geq 1}$ in $\left(D\left(\mathbb{R}_{+}, H^{-s}\right)\right)^{2}$.

Proposition 7.6. Both sequences $\left(U^{N}\right)_{N \geq 1}$ and $\left(V^{N}\right)_{N \geq 1}$ are tight in $D\left(\mathbb{R}_{+}, H^{-s}\right)$.

Proof. We only establish the tightness of $\left(U^{N}\right)_{N \geq 1}$ by showing that the conditions (T1) and (T2) of Proposition 6.7 are satisfied.

The condition (T1) is obtained by using the Proposition 7.5 and applying an argument similar to that of the proof of (T1) in Theorem 6.6.

- Proof of (T2). we have

$$
\begin{aligned}
U_{t}^{N} & =U_{0}^{N}+\beta \int_{0}^{t}\left(G_{r}^{S, I, N}\right)^{*} Z^{N} d r-\beta \int_{0}^{t}\left(G_{r}^{I, N}\right)^{*} U_{r}^{N} d r-\beta \int_{0}^{t}\left(G_{r}^{S}\right)^{*} V_{r}^{N} d r+\widetilde{M}_{t}^{\prime N, \varphi} \\
& =U_{0}^{N}+\beta \int_{0}^{t} \Gamma_{r}^{N} d r+\widetilde{M}_{t}^{N, \varphi} \text { with } \Gamma_{r}^{N}=\left(G_{r}^{S, I, N}\right)^{*} Z^{N}-\left(G_{r}^{I, N}\right)^{*} U_{r}^{N}-\left(G_{r}^{S}\right)^{*} V_{r}^{N} .
\end{aligned}
$$

We want to prove that $\forall T>0, \forall \varepsilon_{1}, \varepsilon_{2}>0, \exists \delta>0, N_{0} \geq 1$ such that for any family of stopping times $\left(\tau^{N}\right)_{N}$ with $\tau^{N} \leq T$

$$
\begin{aligned}
& \sup _{\substack{N \geq N_{0} \\
\delta \geq \theta}} \mathbb{P}\left(\left\|\int_{\tau^{N}}^{\tau^{N}+\theta} \Gamma_{r}^{N} d r\right\|_{H^{-s}}>\varepsilon_{1}\right)<\varepsilon_{2}, \\
& \sup _{\substack{N \geq N_{0} \\
\delta \geq \theta}} \mathbb{P}\left(\left\|\widetilde{M}_{\left(\tau^{N}+\theta\right)}^{\prime N}-\widetilde{M}_{\tau^{N}}^{\prime N}\right\|_{H^{-s}}>\varepsilon_{1}\right)<\varepsilon_{2} .
\end{aligned}
$$

-(7.10) is proved by using an argument similar to that of the proof of (T2) in Proposition 6.15 - Proof of (7.9). Let $T>0, l \in \mathbb{R}_{+} \backslash[0,1], \varepsilon_{1}, \varepsilon_{2}>0$, we find $\delta>0, N_{0} \geq 1$ such that $\delta+\tau^{N} \leq l T$ and

$$
\sup _{\substack{N \geq N_{0} \\ \delta \geq \theta}} \mathbb{P}\left(\left\|\int_{\tau^{N}}^{\tau^{N}+\theta} \Gamma_{r}^{N} d r\right\|_{H^{-s}}>\varepsilon_{1}\right)<\varepsilon_{2} .
$$


We have $\left\|\int_{\tau^{N}}^{\tau^{N}+\theta} \Gamma_{r}^{N} d r\right\|_{H^{-s}} \leq \int_{\tau^{N}}^{\left(\tau^{N}+\theta\right)}\left\|\Gamma_{r}^{N}\right\|_{H^{-s}} d r \leq \theta \sup _{0 \leq r \leq l T}\left\|\Gamma_{r}^{N}\right\|_{H^{-s}}$.

Thus (7.9) will follows from $\sup _{N \geq 1} \mathbb{E}\left(\sup _{0 \leq r \leq l T}\left\|\Gamma_{r}^{N}\right\|_{H^{-s}}\right)<C$ in view of the last inegality, which in turn follows readilly form Lemma 8.2 below, Remark 7.2 and Propositions 4.1 and 7.5, combined with the fact that Corollary 6.20 remains true for $\mu_{t}=\mu$. So (T2) is established.

An easy adaptation of the proof of Lemmas 6.25 and 6.26 yields respectively the next two Lemmas.

Lemma 7.7. For any $t \geq 0, \varphi \in H^{2}\left(\mathbb{T}^{2}\right)$, as $N \longrightarrow \infty$,

$$
\int_{0}^{t} \mathbb{E}\left(\left\|\left(G_{r}^{I, N}-G_{r}^{I}\right) \varphi\right\|_{H^{s}}^{2}\right)^{\frac{1}{2}} d t \longrightarrow 0 .
$$

Lemma 7.8. For any $t \geq 0, \varphi \in H^{s}\left(\mathbb{T}^{2}\right)$, as $N \longrightarrow \infty$,

$$
\int_{0}^{t} \mathbb{E}\left(\left\|\left(G_{r}^{S, I, N}-G_{r}^{S, I}\right) \varphi\right\|_{H^{s}}^{2}\right)^{\frac{1}{2}} d t \longrightarrow 0 .
$$

From Proposition 7.6, we deduce that the sequence $\left(U^{N}, V^{N}\right)_{N}$ is tight in $\left(D\left(\mathbb{R}_{+}, H^{-s}\right)\right)^{2}$, so there exists a subsequence still denoted $\left(U^{N}, V^{N}\right)_{N}$, which converges in law in $\left(D\left(\mathbb{R}_{+}, H^{-s}\right)\right)^{2}$ towards $(U, V)$. Moreover from Proposition 7.4, we deduce that $(U, V) \in\left(C\left(\mathbb{R}_{+}, H^{-s}\right)\right)^{2}$, thus we end the proof of Theorem 7.3 as follows.

Proposition 7.9. The pair $(U, V)$ is the unique solution in $\left(C\left(\mathbb{R}_{+}, H^{-s}\right)\right)^{2}$ of the system formed by equations (7.5) and (7.6).

Proof. By adapting the proof of Proposition 6.27 we obtain the following results

$$
\begin{aligned}
& \int_{0}^{t}\left(U_{r}^{N}, G_{r}^{I, N} \varphi\right) d r \stackrel{L}{\rightarrow} \int_{0}^{t}\left(U_{r}, G_{r}^{I} \varphi\right) d r \\
& \int_{0}^{t}\left(Z_{r}^{N}, G_{r}^{S, I, N} \varphi\right) d r \stackrel{L}{\rightarrow} \int_{0}^{t}\left(Z_{r}, G_{r}^{S, I} \varphi\right) d r .
\end{aligned}
$$

Therefore, with the convergence of the other terms of equations (7.3) and (7.4), we see that the pair $(U, V)$ satisfies the equations (7.5) and (7.6). By adapting the proof of Proposition 6.28 we also see that the system formed by equations (7.5) and (7.6) admits a unique solution in $\left(C\left(\mathbb{R}_{+}, H^{-s}\right)\right)^{2}$.

\section{Appendix}

We first recall that for any $s>0$, the family $\left(\rho_{n_{1}, n_{2}}^{i, s}\right)_{i, n_{1}, n_{2}}$ (as defined in Proposition 2.2) is an orthonormal basis of $H^{s}\left(\mathbb{T}^{2}\right)$.

In this appendix we prove the next two Lemmas.

Lemma 8.1. $\forall x \in \mathbb{T}_{2}$, we have,

$$
\begin{gathered}
\sum_{i, n_{1}, n_{2}}\left(\rho_{n_{1}, n_{2}}^{i, s}(x)\right)^{2}<\infty \text { iff } s>1, \\
\sum_{i, n_{1}, n_{2}}\left(\nabla \rho_{n_{1}, n_{2}}^{i, s}(x)\right)^{2}<\infty \text { iff } s>\mathcal{L} .
\end{gathered}
$$

Proof. As for any $x \in \mathbb{T}^{2}, i \in[|1,8|], 0 \leq\left(f_{n_{1}, n_{2}}^{i}(x)\right)^{2} \leq 4$,

$$
\begin{aligned}
& \sum_{i, n_{1}, n_{2}}\left(\rho_{n_{1}, n_{2}}^{i, s}(x)\right)^{2}=\sum_{i, n_{1}, n_{2}} \frac{\left(f_{n_{1}, n_{2}}^{i}\right)^{2}(x)}{\left(1+\gamma \pi^{2}\left(n_{1}^{2}+n_{2}^{2}\right)\right)^{s}} \text { and, } \\
& \sum_{i, n_{1}, n_{2}}\left(\nabla \rho_{n_{1}, n_{2}}^{i, s}(x)\right)^{2}=\pi^{2} \sum_{i=1}^{4} \sum_{n_{1}>0, n_{2}>0, \text { even }} \frac{n_{1}^{2}+n_{2}^{2}}{\left(1+\gamma \pi^{2}\left(n_{1}^{2}+n_{2}^{2}\right)\right)^{s}}\left(f_{n_{1}, n_{2}}^{i}\right)^{2}(x)
\end{aligned}
$$


So

$$
+\sum_{n_{1}>0, \text { even }} \frac{\pi^{2} n_{1}^{2}\left[\left(f_{n_{1}, 0}^{6}\right)^{2}(x)+\left(f_{n_{1}, 0}^{5}\right)^{2}(x)\right]}{\left(1+\gamma \pi^{2} n_{1}^{2}\right)^{s}}+\sum_{n_{2}>0, \text { even }} \frac{\pi^{2} n_{2}^{2}\left[\left(f_{n_{1}, 0}^{8}\right)^{2}(x)+\left(f_{n_{1}, 0}^{7}\right)^{2}(x)\right]}{\left(1+\gamma \pi^{2} n_{2}^{2}\right)^{s}}
$$

$$
\begin{gathered}
\sum_{i, n_{1}, n_{2}}\left\{\left(\rho_{n_{1}, n_{2}}^{i, s}(x)\right)^{2} \leq 1+16 \sum_{n_{1}>0, n_{2}>0, \text { even }} \frac{1}{\left(1+\gamma \pi^{2}\left(n_{1}^{2}+n_{2}^{2}\right)\right)^{s}}+8 \sum_{i=1}^{2} \sum_{n_{i}>0, \text { even }} \frac{1}{\left(1+\gamma \pi^{2} n_{i}^{2}\right)^{s}}\right. \text { and, } \\
\sum_{i, n_{1}, n_{2}}\left(\nabla \rho_{n_{1}, n_{2}}^{i, s}(x)\right)^{2} \leq 16 \pi^{2} \sum_{n_{1}>0, n_{2}>0, \text { even }} \frac{n_{1}^{2}+n_{2}^{2}}{\left(1+\gamma \pi^{2}\left(n_{1}^{2}+n_{2}^{2}\right)\right)^{s}}+8 \pi^{2} \sum_{i=1}^{2} \sum_{n_{i}>0, \text { even }} \frac{n_{i}^{2}}{\left(1+\gamma \pi^{2} n_{i}^{2}\right)^{s}}
\end{gathered}
$$

Hence we see that:

$-\sum_{i, n_{1}, n_{2}}\left(\rho_{n_{1}, n_{2}}^{i, s}(x)\right)^{2}<\infty$ provided the series $\sum_{n_{1}>0, n_{2}>0} \frac{1}{\left(1+\gamma \pi^{2}\left(n_{1}^{2}+n_{2}^{2}\right)\right)^{s}} ; \sum_{n_{1}>0} \frac{1}{\left(1+\gamma \pi^{2} n_{1}^{2}\right)^{s}}$ and $\sum_{n_{2}>0} \frac{1}{\left(1+\gamma \pi^{2} n_{2}^{2}\right)^{s}}$ converge.

$-\sum_{i, n_{1}, n_{2}}\left(\nabla \rho_{n_{1}, n_{2}}^{i, s}(x)\right)^{2}<\infty$ provided the series $\sum_{n_{1}>0, n_{2}>0} \frac{n_{1}^{2}+n_{2}^{2}}{\left(1+\gamma \pi^{2}\left(n_{1}^{2}+n_{2}^{2}\right)\right)^{s}} ; \sum_{n_{1}>0} \frac{n_{1}^{2}}{\left(1+\gamma \pi^{2} n_{1}^{2}\right)^{s}}$ and $\sum_{n_{2}>0} \frac{n_{2}^{2}}{\left(1+\gamma \pi^{2} n_{2}^{2}\right)^{s}}$ converge.

\section{- Convergence of the series}

1) Convergence of $\sum_{n_{1}>0, n_{2}>0} \frac{n_{1}^{2}+n_{2}^{2}}{\left(1+\gamma \pi^{2}\left(n_{1}^{2}+n_{2}^{2}\right)\right)^{s}}$

It is so easy to see that $\sum_{n_{1} \geq 1, n_{2} \geq 1} \frac{n_{1}^{2}+n_{2}^{2}}{\left(1+\gamma \pi^{2}\left(n_{1}^{2}+n_{2}^{2}\right)\right)^{s}}$ and $\int_{1}^{+\infty} \int_{1}^{+\infty} \frac{x^{2}+y^{2}}{\left(1+\gamma \pi^{2}\left(x^{2}+y^{2}\right)\right)^{s}} d x d y$ are of the same type (either convergent or divergent), and the latter is of the same type as

$$
\int_{1}^{+\infty} \frac{r^{3}}{\left(1+\gamma \pi^{2} r^{2}\right)^{s}} d r \leq \frac{1}{\gamma^{s} \pi^{2 s}} \int_{1}^{+\infty} r^{3-2 s} d r \text { and } \int_{1}^{+\infty} r^{3-2 s} d r<\infty \text { iff } \mathrm{s}>2 .
$$

Thus $\sum_{n_{1} \geq 1, n_{2} \geq 1} \frac{n_{1}^{2}+n_{2}^{2}}{\left(1+\gamma \pi^{2}\left(n_{1}^{2}+n_{2}^{2}\right)\right)^{s}}$ converges iff $\mathrm{s}>2$.

2) By the same argument as previously $\sum_{n_{1}>0, n_{2}>0} \frac{1}{\left(1+\gamma \pi^{2}\left(n_{1}^{2}+n_{2}^{2}\right)\right)^{s}}$ converges for $\mathrm{s}>1$.

3) By the comparison criterion the series $\sum_{n_{1}>0} \frac{1}{\left(1+\gamma \pi^{2} n_{1}^{2}\right)^{s}}$ and $\sum_{n_{2}>0} \frac{1}{\left(1+\gamma \pi^{2} n_{2}^{2}\right)^{s}}$ converge for $s>\frac{1}{2}$.

4) By the comparison criterion the series $\sum_{n_{1}>0} \frac{n_{1}^{2}}{\left(1+\gamma \pi^{2} n_{1}^{2}\right)^{s}}$ and $\sum_{n_{2}>0} \frac{n_{2}^{2}}{\left(1+\gamma \pi^{2} n_{2}^{2}\right)^{s}}$ converge for $s>\frac{3}{2}$

Lemma 8.2. Under the assumption (H2), for any $t \geq 0$, we have

$$
\sup _{x}\left\|\frac{K(x, .)}{\int_{\mathbb{T}^{2}} K\left(x^{\prime}, .\right) \mu_{t}\left(d x^{\prime}\right)}\right\|_{H^{3}}^{2}<\infty
$$

Proof. We refer to Proposition 2.4 above. $H^{3}\left(\mathbb{T}^{2}\right) \subset C^{1}\left(\mathbb{T}^{2}\right)$.

If we let $\operatorname{support}\{K(x, \cdot)\}=A(x)$, we will have

$$
\left\|\frac{K(x, .)}{\int_{\mathbb{T}^{2}} K\left(x^{\prime}, .\right) \mu_{t}\left(d x^{\prime}\right)}\right\|_{H^{3}}^{2}=\sum_{|\eta| \leq 3} \int_{A(x)}\left|D^{\eta} \frac{K(x, y)}{\int_{A(y)} K\left(x^{\prime}, y\right) \mu_{t}\left(d x^{\prime}\right)}\right|^{2} d y .
$$

Now letting $w_{t}(x, y)=\frac{K(x, y)}{\int_{A(y)} K\left(x^{\prime}, y\right) \mu_{t}\left(d x^{\prime}\right)}$,

since from Lemma 6.2 , for any $x \in \mathbb{T}^{2}, K(x,.) \in H^{3} \subset C^{1}\left(\mathbb{T}^{2}\right)$, one has

$$
\begin{aligned}
& \frac{\partial w_{t}}{\partial y_{1}}(x, y)=\frac{\frac{\partial K}{\partial y 1}(x, y)}{\int_{\mathbb{T}_{2}} K(u, y) \mu_{t}(d u)}-\frac{K(x, y) \int_{A(y)} \frac{\partial K}{\partial y_{1}}(u, y) \mu_{t}(d u)}{\left(\int_{A(y)} K(u, y) \mu_{t}(d u)\right)^{2}} . \\
& \frac{\partial^{2} w_{t}}{\partial y_{2} y_{1}}(x, y)=\frac{\frac{\partial^{2} K}{\partial y_{2} y_{1}}(x, y)}{\int_{A(y)} K(u, y) \mu_{t}(d u)}-\frac{\frac{\partial K}{\partial y_{1}}(x, y) \int_{A(y)} \frac{\partial K}{\partial y_{2}}(u, y) \mu_{t}(d u)}{\left(\int_{A(y)} K(u, y) \mu_{t}(d u)\right)^{2}}
\end{aligned}
$$




$$
\begin{gathered}
-\frac{\frac{\partial K}{\partial y_{2}}(x, y) \int_{A(y)} \frac{\partial K}{\partial y_{1}}(u, y) \mu_{t}(d u)+K(x, y) \int_{A(y)} \frac{\partial^{2} K}{\partial y_{2} y_{1}}(u, y) \mu_{t}(d u)}{\left(\int_{A(y)} K(u, y) \mu_{t}(d u)\right)^{2}} \\
+2 \frac{K(x, y) \int_{A(y)} \frac{\partial K}{\partial y_{1}}(u, y) \mu_{t}(d u) \int_{A(y)} \frac{\partial K}{\partial y_{2}}(u, y) \mu_{t}(d u)}{\left(\int_{A(y)} K(u, y) \mu_{t}(d u)\right)^{3}} . \\
\frac{\partial^{3} w_{t}}{\partial y_{1} y_{2} y_{1}}(x, y)=\frac{\frac{\partial^{3} K}{\partial y_{1} y_{2} y_{1}}(x, y)}{\int_{A(y)} K(u, y) \mu_{t}(d u)}-2 \frac{\frac{\partial^{2} K}{\partial y_{2} y_{1}}(x, y) \int_{A(y)} \frac{\partial K}{\partial y_{1}}(u, y) \mu_{t}(d u)}{\left(\int_{\mathbb{T}_{2}} K(u, y) \mu_{t}(d u)\right)^{2}} \\
-\frac{\frac{\partial^{2} K}{\partial y_{1} y_{1}}(x, y) \int_{A(y)} \frac{\partial K}{\partial y_{2}}(u, y) \mu_{t}(d u)+2 \frac{\partial K(x, y)}{\partial y_{1}} \int_{A(y)} \frac{\partial^{2} K}{\partial y_{1} y_{2}}(u, y) \mu_{t}(d u)}{\left(\int_{A(y)} K(u, y) \mu_{t}(d u)\right)^{2}} \\
+4 \frac{\frac{\partial K}{\partial y_{1}}(x, y) \int_{A(y)} \frac{\partial K}{\partial y_{2}}(u, y) \mu_{t}(d u) \int_{A(y)} \frac{\partial K}{\partial y_{1}}(z, y) \mu_{t}(d u)}{\left(\int_{A(y)} K(u, y) \mu_{t}(d u)\right)^{3}} \\
-\frac{\frac{\partial K}{\partial y_{2}}(x, y) \int_{A(y)} \frac{\partial^{2} K}{\partial y_{2} y_{1}}(u, y) \mu_{t}(d u)+K(x, y) \int_{A(y)} \frac{\partial^{3} K}{\partial y_{1} y_{2} y_{1}}(u, y) \mu_{t}(d u)}{\left(\int_{A(y)} K(u, y) \mu_{t}(d u)\right)^{2}} \\
+2 \frac{\int_{A(y)} \frac{\partial K}{\partial y_{1}}(u, y) \mu_{t}(d u)\left[\frac{\partial K}{\partial y_{2}}(x, y) \int_{\mathbb{T}_{2}} \frac{\partial K}{\partial y_{1}}(u, y) \mu_{t}(d u)+2 K(x, y) \int_{A(y)} \frac{\partial^{2} K}{\partial y_{2} y_{1}}(u, y) \mu_{t}(d u)\right]}{\left(\int_{A(y)} K(u, y) \mu_{t}(d u)\right)^{3}} \\
+2 \frac{K(x, y) \int_{A(y)} \frac{\partial^{2} K}{\partial y_{1} y_{1}}(u, y) \mu_{t}(d u) \int_{A(y)} \frac{\partial K}{\partial y_{2}}(u, y) \mu_{t}(d u)}{\left(\int_{A(y)} K(u, y) \mu_{t}(d u)\right)^{3}} \\
+6 \frac{K(x, y)\left(\int_{A(y)} \frac{\partial K}{\partial y_{1}}(u, y) \mu_{t}(d u)\right)^{2} \int_{A(y)} \frac{\partial K}{\partial y_{2}}(u, y) \mu_{t}(d u)}{\left(\int_{A(y)} K(u, y) \mu_{t}(d u)\right)^{4}}
\end{gathered}
$$

On the other hand if we let $\mathcal{C}_{1}=\left\{2 k^{\prime}\left(\|x-y\|^{2}\right), 4 k^{\prime \prime}\left(\|x-y\|^{2}\right), 8 k^{(3)}\left(\|x-y\|^{2}\right)\right\}$ and $\mathcal{C}_{2}=\left\{\left(x_{1}-y_{1}\right)^{n}\left(x_{2}-y_{2}\right)^{m},(n, m) \in\{0,1,2,3\}^{2}\right\}, \forall|\eta| \leq 3, D^{\eta} K(x, y)=D^{\eta} k\left(\|x-y\|^{2}\right)$ is written as the sum of the products of elements of $\mathcal{C}_{1}$ and $\mathcal{C}_{2}$. Thus as from assumption (H2), $\forall|\eta| \leq 3,\left|k^{(|\eta|)}\right|$ is bounded in $\mathbb{R}_{+}, \forall|\eta| \leq 3, x \in \mathbb{T}^{2}, D^{\eta} K(x, y)$ is bounded by a constant independent of $x$. Now since $\forall y \in \mathbb{T}^{2}, \int_{\mathbb{T}^{2}} K(u, y) \mu_{t}^{N}(d u)=\int_{\mathbb{T}^{2}} K(u, y) f(t, u)(d u)$ is lower bounded by a constant independent of y and $f(t,.) \leq \delta_{2}$ then we deduce from the above calculations that $\sum_{|\eta| \leq 3} \int_{A(x)}\left|D^{\eta} \frac{K(x, y)}{\int_{A(y)} K\left(x^{\prime}, y\right) \mu_{r}\left(d x^{\prime}\right)}\right|^{2} d y$ is bounded by a constant independent of $x$. Hence the result.

Funding: Alphonse Emakoua was supported by EMS Simons For Africa and an Eiffel scholarship program of excellence, and the two other authors by their respective university.

\section{References}

[1] R.A. Adams. Sobolev Spaces. Academic Press, 1975.

[2] D. Aldous . Stopping times and tightness. The Annals of Probability 6(2), 335-340, 1978

[3] L.J.S. Allen, B.M Botlker, Y. lou and A.L. Nevai Asymptotic profiles of the steady states for an SIS epidemic reaction diffusion model, Discrete Contin. Dyn. Syst. Ser. A 21, 1-20, 2008.

[4] H. Andersson, T. Britton. stochastic epidemic models and their statistical analysis. Springer Lecture Notes in Statistics. Springer, New York (2000)

[5] Hajer Bahouri, Jean-Yves Chemin and Raphaël Danchin. Fourier Analysis and Nonlinear Partial Differential Equations, Springer (2011). 
[6] P. Billingsley, Convergence of Probability Measures, 2nd edn. (Wiley, New York, 1999)

[7] Douglas Blount. Density-dependant limits for a nonlinear reaction diffusion models, The Anals of Probability, vol. 22, N0.4 2040-2070, 1994

[8] Tom. Britton, Etienne Pardoux. Stochastic epidemic in a homogeneous community, Part I of stochastic epidemic models with inference. T. Britton, E. Pardoux. eds, Lecture Notes in Mathematics 2225, pp. 1-120 Springer 2019

[9] Axel Bücher and Ivan Kojadinovic, A note on conditional versus joint unconditional weak convergence in bootstrap consistency results, arXiv:1706.01031v4 [math.ST] 1 Mar 2018

[10] Jie Yen Fan, Kais Hamza, Peter Jagers, and Fima Klebaner. Spde limits for the age structure of a population, arxiv1702.08592v1

[11] Loukas Grafakos. Classical fourier analysis 2nd. ed Springer. 2008.

[12] J. Jacod and A.N. Shiryaev. Limit Theorems for Stochastic Processes. Springer-Verlag, Berlin, 1987.

[13] Joffe and M. Metivier. Weak Convergence of Sequences of Semimartingales with Applications to Multitype Branching Processes, Advances in Applied Probability, Vol. 18, No. 1, pp. 20-65, 1986

[14] Olav Kallenberg Lectures on random measures, Institute of Statistics Mimeo Series No. 963 November, 1974

[15] P. Kotelenez .High density limit théorems for non linear chemical reactions with diffusion. Proba Th.Rel.Fields 78,11-37, 1998

[16] S. Méléard. Convergence of the fluctuations for interacting diffusions with jumps associated with Boltzmann equation. Stochastics and Stochastics Reports, 63 :195-225, 1998.

[17] Sylvie Méléard et Sylvie Roelly: Sur les convergences étroite ou vague de processus à valeurs mesures. Comptes rendus de l'académie des Sciences de Paris Sér. 1, 317:785-788, 1993.

[18] M. Métivier. Convergence faible et principe d'invariance pour des martingales à valeurs dans des espaces de Sobolev. Annales de l'IHP, 20(4) :329-348, 1984.

[19] M. Metivier and S. Nakao, Equivalent conditions for the tightness of a sequence of continuous Hilbert valued martingales, Nagoya Math. J. 106, 113-119, 1987.

[20] Étienne Pardoux. Equations aux dérivées Partielles stochastiques non liiéaires monotones. Etudes des solutions fortes de type Itô, thèse 1975.

[21] Étienne Pardoux. Moderate Deviations and Extinction of an Epidemic. Election. J. Probab 25, paper $\mathrm{N}^{\circ} 25,1-27,2020$

[22] Étienne Pardoux. Probabilistic Models of Population Evolution, Scaling Limits, Genealogies and Interactions, Springer 2016.

[23] Étienne Pardoux. Stochastic Partial Differential Equations and Filtering of Diffusion Processes. Stochastics Vol. 3. pp 127-167, 1979.

[24] Sylvie Roelly-Coppoletta. A criterion of convergence of measure-valued processes: application to measure branching processes Stochastics, 17 :43-65, 1985.

[25] M.E Taylor. Partial differential equations III Nonlinear equations. Springer 1991

[26] Viet Chi Tran Modèles particulaires stochastiques pour des problèmes d'évolution adaptative et pour l'approximation de solutions statistiques, thèse 2006 